Aus der Klinik für Pädiatrische Onkologie und Hämatologie

Universitätsklinikum des Saarlandes,

Homburg/Saar

(Direktor: Univ.-Professor Dr. med. Norbert Graf)

\title{
An innovative mathematical analysis of routine MRI scans in patients with glioblastoma using DoctorEye
}

Dissertation zur Erlangung des Grades eines Doktors der Medizin

der Medizinischen Fakultät

der UNIVERSITÄT DES SAARLANDES

2013

vorgelegt von: Jonathan Nicolas Zepp

geboren am 15.01.1986 in Mainz 



\section{Contents}

1 Abstract

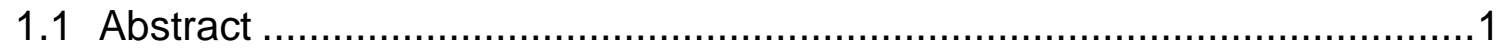

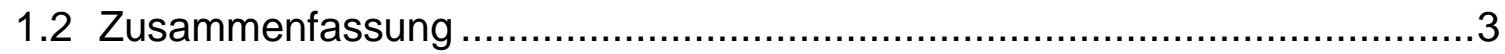

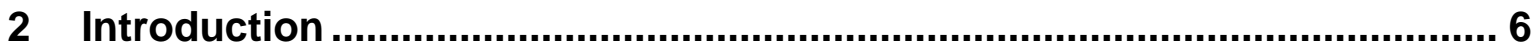

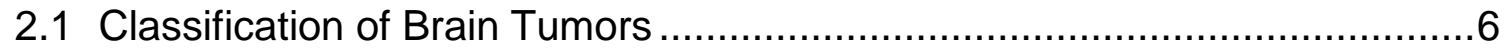

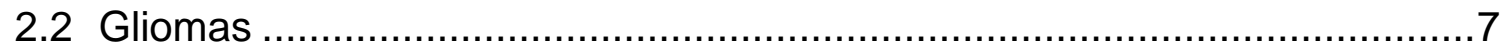

2.3 Clinical Manifestation of Malignant Gliomas ...........................................

2.4 Diagnostic Procedures of Brain Tumors ..............................................

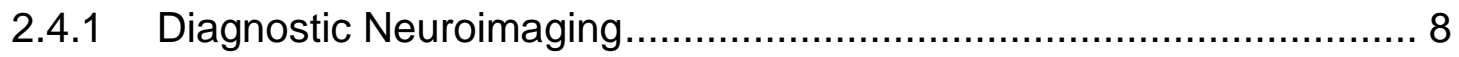

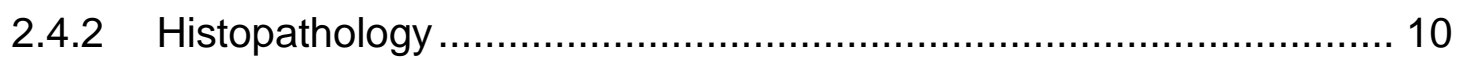

2.4.3 Molecular Genetics ............................................................... 11

2.5 Treatment and Prognosis of Malignant Gliomas .....................................12

2.5.1 Initial surgical Approach........................................................ 12

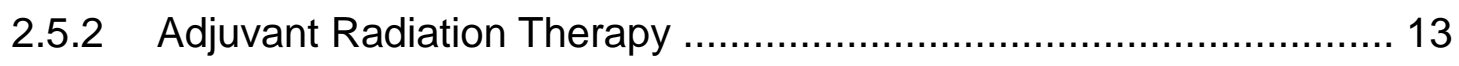

2.5.3 Adjuvant Chemotherapy ........................................................ 13

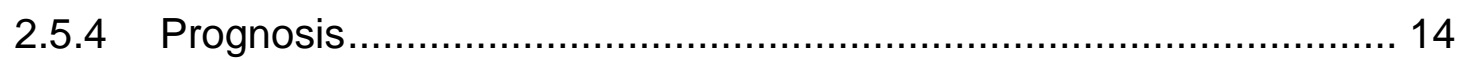

2.6 Assessment of Response and Progression and its Limitations ..................14

2.6.1 MacDonald Criteria ................................................................ 15

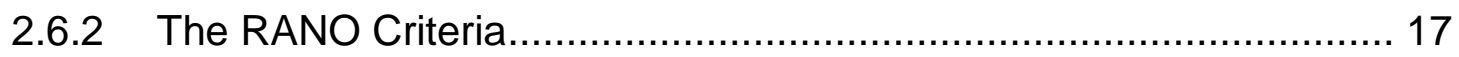

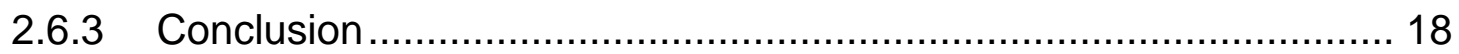

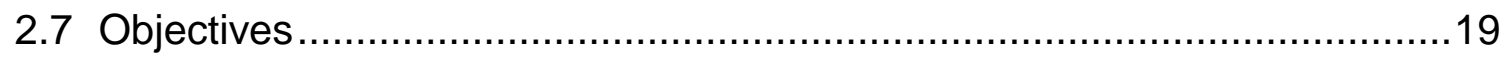

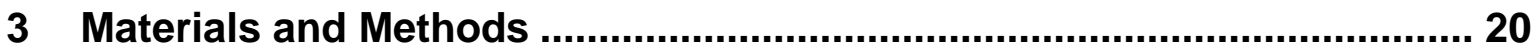

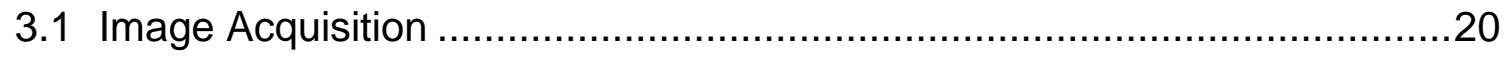

3.2 Investigation Process and Equipment Technology ……...........................23

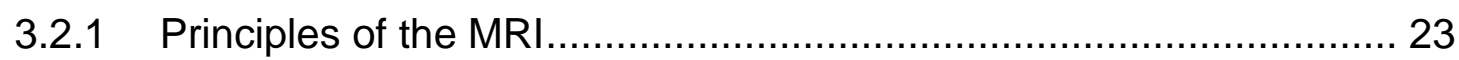

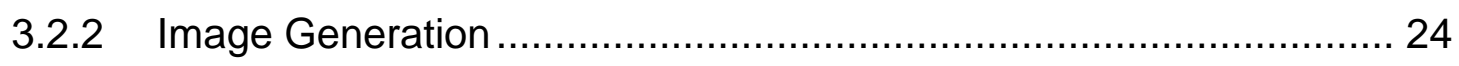

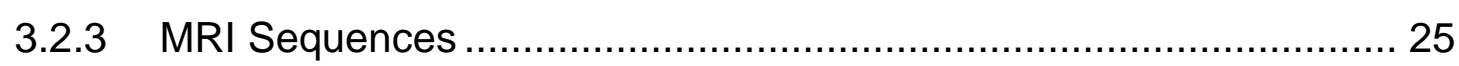

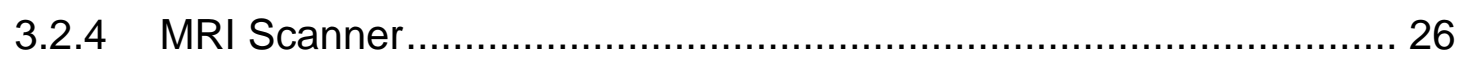




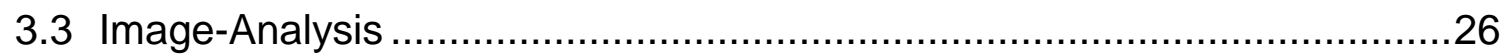

3.3.1 Background on DoctorEye (4.7) .............................................. 27

3.3.2 Selected Segmentation Method - The Magic Wand Algorithm ......... 29

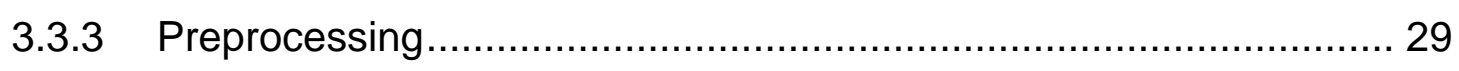

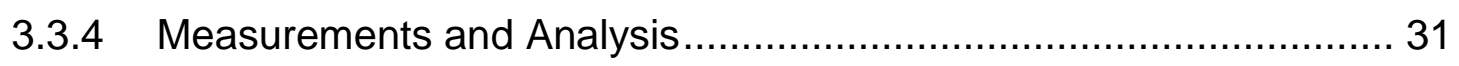

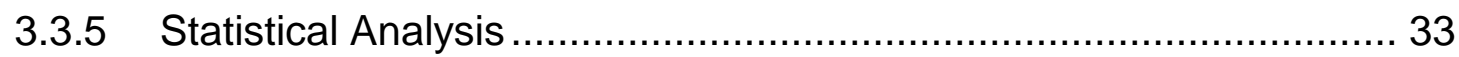

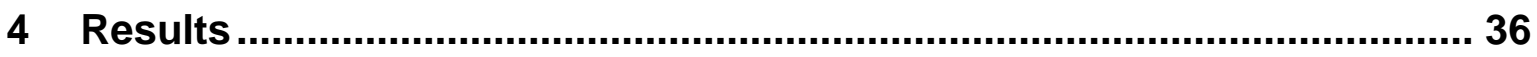

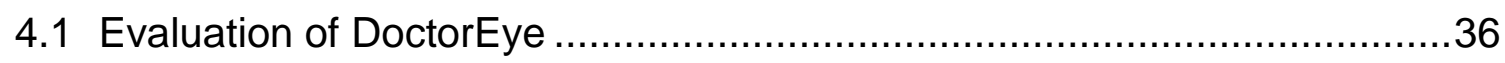

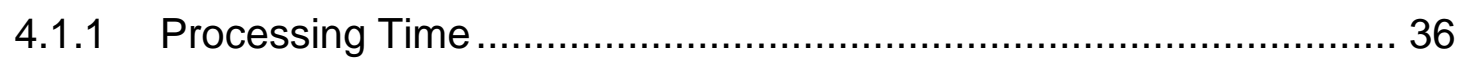

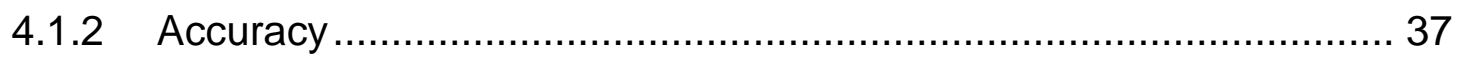

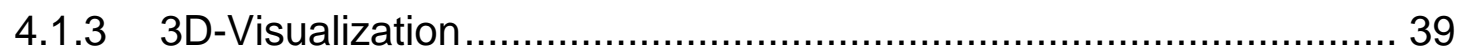

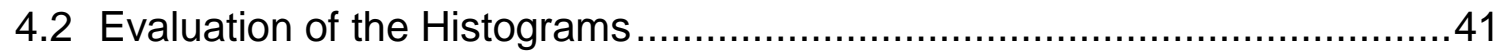

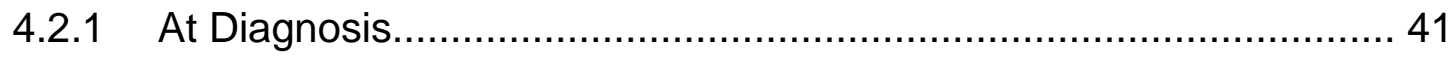

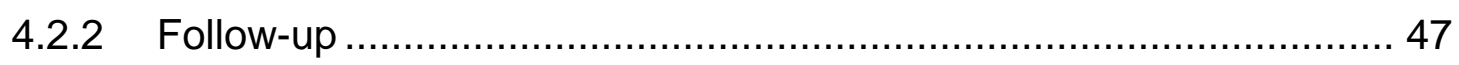

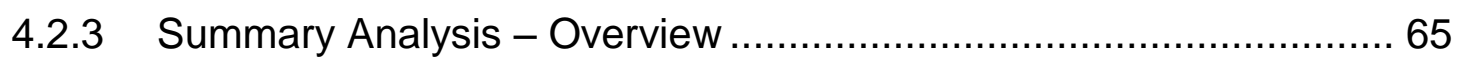

4.2.4 Volume Changes during Follow-up.............................................. 67

4.2.5 Assessment of Progression and Response .................................. 70

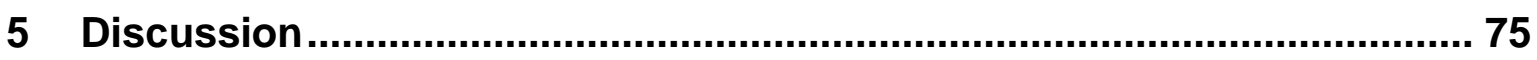

5.1 Evaluation of Software and Segmentation Methods …............................75

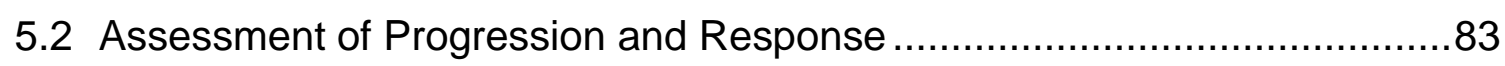

5.2.1 Different Imaging Modalities ……………............................... 94

5.2.2 Imaging Phenomena: Pseudoprogression, Radiation Necrosis and

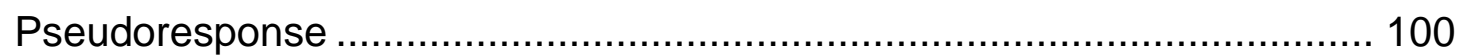

5.2.3 Volumetric Measurements ......................................................... 103

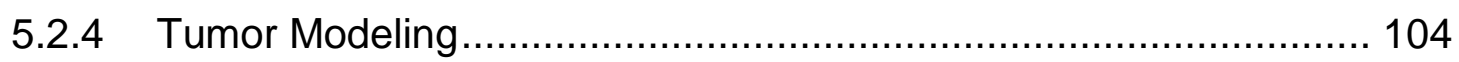

5.3 Limitations and Perspectives ...........................................................105 


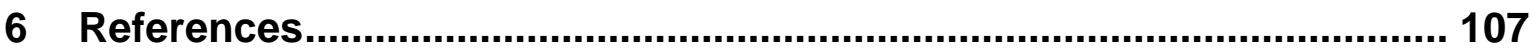

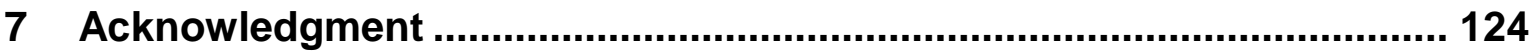

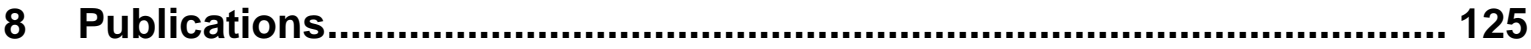

9 Appendix

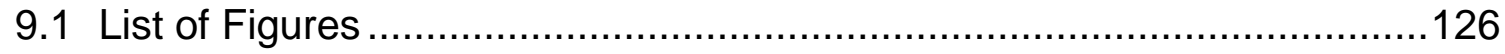

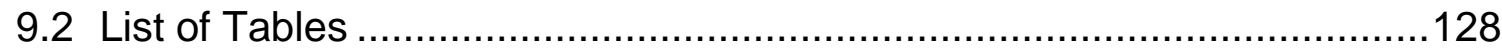

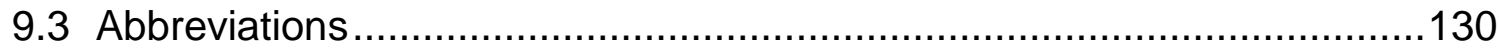

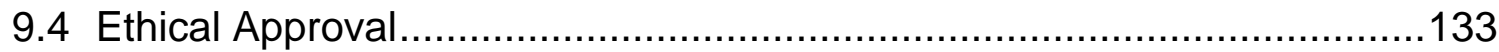

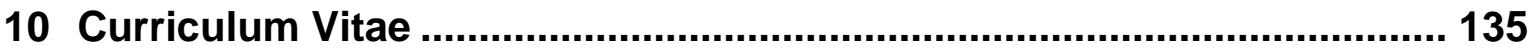




\section{Abstract}

\subsection{Abstract}

Glioblastoma multiforme belongs to the group of gliomas and represents the most malignant entity of all primary brain tumors in adults. Despite recent diagnostic and therapeutic achievements the glioblastoma has the poorest prognosis of all primary brain tumors. Improving the initial diagnosis and the assessment of response to treatment in malignant gliomas, while avoiding invasive methods as much as justifiable, is one major aspect actual research is focusing on. Imaging studies are used to calculate tumor volume and to define vital, necrotic and cystic areas within a tumor. Since the subjective, visual interpretation of MRI is based on qualitative observation of variation in signal intensity, a correlation of signal intensities with histological features of a tumor in this manner is not possible. The need for an objective method for the reliable description of a tumor and the interpretation of follow-up studies in single patients and their collective was the inspiration to the concept of the present study.

In the proposed work histograms of signal intensities from delineated areas in MRI scans served as a method of quantitative data acquisition. Using the image analysis software DoctorEye, tumors and other pathologic tissues as necrosis or edema could easily be rendered, while at the same time histograms of the signal intensities within a tumor, as well as mean and median signal intensities were calculated. Additionally this technique enabled us to precisely calculate the volumes of the different investigated tissues (active tumor, necrosis and edema) and to assess their development during the course of disease. To the best of my knowledge there was no data available that uses histograms of signal intensities of MRI for the characterization of glioblastoma, when the proposed study was initiated.

In total more than 22.000 DICOM-files based on routine-MRI scans, from 33 anonymized patients suffering from suspected glioblastoma, have been analyzed. 
These data included T1, T1 with gadolinium contrast enhancement, T2 and T2Flair sequences, representing another novelty, as in the common criteria most often only the assessment of the $\mathrm{T} 1$ with gadolinium contrast enhancement sequence in MR images is suggested. The data of the histograms were uploaded to Microsoft Office Excel 2007, to perform further statistics, including the normalization of the data. In addition to the comparison of the distribution of the signal intensities, focus was laid on the calculation and interpretation of the respective maximum values and the average signal intensities of the histograms and their development over time. In nine of the 33 patients a complete follow-up could be analyzed, referring to DICOM data-sets at diagnosis, after surgery and after radio- and chemotherapy.

Since the histograms of the different pathologic tissues vary in all sequences, the measurement of volumes and the segmentation of the tumor in the various MR sequences offer an optimal way to objectively distinguish between active tumor tissue, necrosis and edema. At the time of diagnosis the histogram of all glioblastoma showed a bimodal distribution of signal intensities in the T1 with gadolinium contrast enhancement sequence. Such similarities of the graphical shape are seen during follow-up in individual patients as well.

It can be assumed that by using combinations of histograms from different sequences the tumor can be described in a much better way than by calculating solely the tumor volume. With the proposed method one can define necrotic areas and active tumor, and simultaneously calculate their volume for any point of time. By standardizing the average signal intensity, relatively to the signal intensities of the corresponding cerebrospinal fluid, it was possible to demonstrate a clear difference between tumor and necrosis at different points of time and in different sequences. The results of the present study lead to the conclusion that the use of these histograms is an innovative way to gain new, tumor-specific information, without performing additional examinations on the patient. This method can be a complementary diagnostic tool to distinguish between different intracranial lesions, and to assess the response to treatment of glioblastoma or its progression. 
Nevertheless, a standardized method for image acquisition is needed to apply this method center-independent and to achieve a higher comparability between the results. Furthermore, the proposed segmentation algorithm too time consuming to be applied in routine clinical practice. If these restrictions will be resolved, this method can constitute a simple, effective tool for the diagnosis, the assessment of individual and collective courses of disease and the modeling of tumor growth and response to treatment.

\subsection{Zusammenfassung}

Eine innovative mathematische Analyse von Routine-MRT-Bildern bei Patienten mit Glioblastom mithilfe von DoctorEye

Das Glioblastom gehört zu der Gruppe der Gliome und stellt die häufigste Entität aller primären Gehirntumoren im Erwachsenenalter dar. Trotz jüngster diagnostischer und therapeutischer Errungenschaften hat das Glioblastom immer noch die schlechteste Prognose aller primären Hirntumoren. Die Verbesserung der Erstdiagnose und die Beurteilung des Ansprechens auf die Behandlung von malignen Gliomen, bei gleichzeitiger, vertretbarer Vermeidung invasiver Methoden, ist ein wichtiger Aspekt auf den sich die aktuelle Forschung konzentriert. Bildgebende Verfahren werden verwendet, um Tumor-Volumina zu berechnen und um zwischen vitalen, nekrotischen und zystischen Tumorarealen zu unterscheiden. Da die subjektive, visuelle Interpretation von MRT-Bildern auf einer qualitativen Beobachtung von Variationen der Signalintensität basiert, ist eine Korrelation von Signalintensitäten mit histologischen Merkmalen eines Tumors auf diesem Wege nicht möglich. Der Mangel an objektivierbaren Methoden zur zuverlässigen Beschreibung von Tumoren und der Interpretation der Follow-up Studien von einzelnen Patienten und deren Kollektiv war Inspiration zu dem Konzept der vorgestellten Studie.

In der vorliegenden Arbeit wurden Histogramme von Signalintensitäten umschriebener Areale in MRT Abbildungen als ein Verfahren zur quantitativen 
Datenerhebung verwendet. Mithilfe der Bildbearbeitungssoftware DoctorEye konnten Tumoren und andere patholgische Gewebeveränderungen, wie Ödeme und Nekrosen, benutzerfreundlich segmentiert und gleichzeitig Histogramme der Signalintensitäten innerhalb eines Tumors, sowie mittlere und mediane Signalintensitäten berechnet werden. Zusätzlich war es möglich mit diesem Verfahren genaue Volumina der einzelnen Gewebetypen (aktiver Tumor, Nekrose und Ödem) zu bestimmen und deren Entwicklung im Krankheitsverlauf zu beurteilen. Nach meinem Kenntnisstand gab es zu dem Zeitpunkt, als die vorliegende Studie initiert wurde, keine vergleichbaren Daten, die Histogramme der Signalintensitäten von MRTs verwendeten, um Glioblastome zu charakterisieren.

Insgesamt wurden über 22.000 DICOM-Dateien, basierend auf Routine-MRTBildern von 33 anonymisierten Patienten mit histologisch gesichertem oder bildmorphologisch verdächtigem Glioblastom, bearbeitet. Diese Bilddateien umfassten die Sequenzen T1 ohne Kontrast, T1 mit Gadolinium-Kontrast, T2 und T2 Flair. Dies ist insofern eine Neuheit, da in den anerkannten Diagnosekriterien meist nur die T1 mit Gadolinium-Kontrast-Sequenzen beurteilt werden. Durch DoctorEye war es möglich die gewonnenen Daten zu extrahieren und in Microsoft Excel $2007 \mathrm{zu}$ transferieren, um dort weitere statistische Untersuchungen anhand der Histogramme, sowie die Normalisierung der Daten durchzuführen. Neben dem Vergleich der Verteilung der Signalintensitäten, lag das Hauptaugenmerk auf der Berechnung und Interpretation der jeweiligen Maximalwerte und der durchschnittlichen Signalintensitäten der Histogramme und deren Entwicklung im zeitlichen Verlauf. Dies geschah für die verschiedenen untersuchten Gewebetypen in jeder MRT-Modalität, zum Zeitpunkt der Diagnose und während der Nachbeobachtungszeit. In 9 der 33 Patienten konnte eine komplette Follow-upStudie rekonstruiert und analysiert werden, unter Bezugnahme auf die DICOMDaten-Sets zum Zeitpunkt der Diagnose, nach Operation und nach Radiochemotherapie. Da sich die Histogramme der verschiedenen pathologischen Gewebeveränderungen in allen Modalitäten unterscheiden, bietet die Messung der Volumina und die Segmentierung des Tumors in den verschiedenen MR 
Sequenzen eine optimale Möglichkeit, aktives Tumor-Gewebe, Nekrose und Ödem objektiv voneinander unterscheiden zu können. Zum Zeitpunkt der Diagnose zeigte das Histogramm aller Glioblastom in T1 mit Gadolinium-Kontrast eine bimodale Verteilung der Signalintensitäten. Solche Übereinstimmungen in der graphischen Form der Histogramme waren auch in der Verlaufsbeobachtung einzelner Patienten gut nachvollziehbar.

Diese Feststellungen lassen vermuten, dass Tumoren wesentlich besser durch die Kombination von Histogrammen verschiedener Modalitäten beurteilt werden können, als durch die alleinige Berechnung des Volumens. Mit der vorgestellten Methode können nekrotische Areale und aktiver Tumor definiert und gleichzeitig das Volumen zum jeweiligen Zeitpunkt berechnet werden. Durch die Standardisierung der durchschnittlichen Signalintensitäten anhand einer Relativierung mit den Signalintensitäten des entsprechenden Liquor cerebrospinalis, ist es gelungen einen Unterschied zwischen den Signalintensitäten von Tumor und Nekrose zu verschiedenen Zeitpunkten und in verschiedenen Modalitäten nachzuweisen. Diese Ergebnisse lassen die Schlussfolgerung zu, dass die Verwendung dieser Histogramme eine innovative Methode ist, um neue, tumorspezifische Informationen zu gewinnen, ohne zusätzliche Untersuchungen am Patienten durchzuführen. Diese Methode kann ein ergänzendes diagnostisches Werkzeug sein, verschiedene intrakranielle Läsion voneinander zu unterscheiden, sowie das Ansprechen eines Glioblastoms auf die Behandlung oder dessen Progress zu beurteilen.

Nichtsdestotrotz ist ein standardisiertes Verfahren zur Bildakquisition vonnöten, um dieses Verfahren auch Zentren übergreifend anwenden zu können und die Ergebnisse vergleichbar zu machen. Desweiteren ist der vorgestellte Segmentierungsalgorithmus noch zu zeitintensiv, um im klinischen Alltag angewendet werden zu können. Werden diese Einschränkungen jedoch behoben könnte das vorgestellte Verfahren ein einfaches und effektives Werkzeug zur Diagnose, Verlaufsbeurteilung und Modellbildung von Tumorwachstum und Therapieansprechen darstellen. 


\section{Introduction}

Brain tumors comprise a group of neoplasms that either originate from brain tissue itself (known as primary brain tumors) or derive from extracranial neoplasms, which have metastasized to the brain (known as secondary brain tumors). The primary brain tumors may evolve from the brain parenchyma, meninges, cranial nerves, and other intracranial structures (the pituitary and pineal glands).

Even if some of the tumor entities are histologically and biologically benign, the constitution of the brain often blurs the differentiation between biologically benign and malignant. Also a small and slow proliferating tumor with little or no signs of malignancy may cause great damage or even prove to be lethal if located in a region where surgery is not possible.

\subsection{Classification of Brain Tumors}

Primary brain tumors are classified by light microscopy according to their predominant cell type, belonging to clearly defined histopathological and clinical features. Since the German pathologist Rudolf Virchow first introduced the term "glioma" in 1860 [91] there have been many attempts of developing a classification system for brain tumors. More than 30 years ago, the international classification of diseases for oncology (ICD-O) was installed and since then serves as an indispensable guideline for the grading of cancers by providing histopathology and mortality data for epidemiological as well as oncological studies [93]. In 1979, the World Health Organization (WHO) published a classification system, which was meant to be the first edition of histological typing of central nervous system (CNS) tumors [170]. Since then it is continuously updated, e.g. by the introduction of immunhistochemistry and genetic profiling as well as clinical signs, symptoms, imaging and many other attributes, aiming at a refined classification [75, 76]. The most recent version of the WHO classification system was published in 2007 and has combined a nomenclature with an implied grading system, so that the actual histological diagnosis correlates with the histological grade of the tumor [93]. A 
higher grade in this classification system implies a higher mitosis rate, more vascular proliferation, giant cells and areas of necrosis (see also chapter 2.4.2 Histopathology). Strikingly, even a histologically relatively well-differentiated glioma (e.g. the grade II astrocytoma) can show a tendency to diffusely infiltrate the brain and to be biologically highly malignant due to the tumor cells capacity to actively migrate along structures within the neuropil. To make matters worse, additionally the gliomas show the inherent capacity to progress to a more malignant phenotype [93].

All these phenomena account for the low survival rates even after all therapeutic modalities have been utilized.

\subsection{Gliomas}

The term "glioma" refers to neoplasms believed to be derived from glial cells, e.g. astrocytes, oligodendrocytes and ependymal cells. Each of this cell type is capable of turning into a certain tumor entity, although many gliomas consist of a composite of them [57]. Among the gliomas themselves, which form $30-50 \%$ of all brain tumors, there are about 50\% high malignant glioblastoma (grade IV), 25\% astrocytomas (grade I-III), 5-18\% oligodendrogliomas and 2-9\% ependymomas [57]. The incidence of gliomas in the United States and in Europe is with 7$11 / 100.000$ citizens significantly higher than in Asia with 2-4/100.000 citizens. Men are affected more often than women [57].

\subsection{Clinical Manifestation of Malignant Gliomas}

The malignant gliomas (WHO grade III and IV) are a group of rapidly progressive brain tumors that are divided into anaplastic gliomas (anaplastic astrocytoma, anaplastic oligodendrogliomas, and anaplastic oligoastrocytoma) and glioblastoma multiforme (GBM), based upon their histopathologic features [93, 157] (see also chapter 2.4.2 - Histopathology).

The clinical manifestations of malignant gliomas depend upon the location as well 
as the size of the lesion and act similar to those produced by other primary and metastatic brain tumors [17].

The Glioma Outcome Project illustrated the spectrum of symptoms produced by malignant gliomas by a series of 565 glioma patients (147 grade III and 418 grade IV) [34]:

- Headache emerged in 53 to $57 \%$ of cases.

- Seizures were found in $56 \%$ of patients with grade III lesions, compared with 23 percent in those with grad IV lesions.

- Other symptoms which were seen in $20 \%$ or more of the patients included memory loss, motor weakness, visual symptoms, language deficit and cognitive and personal changes.

\subsection{Diagnostic Procedures of Brain Tumors}

\subsubsection{Diagnostic Neuroimaging}

To diagnose a patient presenting with signs and symptoms suggesting a brain tumor, neuroradiologic imaging is the most important diagnostic modality. MRI has become the method of choice in the evaluation of neurological disorders, while computer tomography (CT) is used in many cases only in an emergency situation or to clarify specific issues [151]. As of all brain tumors the malignant gliomas grade III and IV have the highest prevalence in older adults and are the ones with the poorest prognosis, it is up to the differential diagnosis to distinguish the variety of tumors, as well as benign lesions from these high-grade gliomas [151].

\subsubsection{Magnetic Resonance Imaging}

$\mathrm{MRI}$ is one alternative of noninvasive tumor imaging. It is characterized by high spatial resolution $(50-500 \mu \mathrm{m})$ and the use of non-ionizing radiation.

Usually a T1 with gadolinium contrast enhancement sequence (Gd-MRI) is the only test needed to suggest a brain tumor, while MRI may also deliver information 
that indicates the specific tumor type:

- Typically malignant gliomas appear hypointense on T1-weighted images, and enhance heterogeneously following contrast infusion. Hence, an enhancing tumor can be distinguished from the surrounding hypointense signal of edema on T1-weighted sequences[161].

- Without regard to the histologic grade, astrocytomas in general tend to show increased T2 and T2 Flair (fluid attenuated inversion recovery) signal intensity (SI), whereas some astrocytomas do not manifest in Gd-MRI [123].

Additionally to permitting visualization of the tumor mass and its relationship to the surrounding normal parenchyma, MRI is also superior to CT for the evaluation of the meninges, subarachnoid space, and posterior fossa, and for the definition of the vascular distribution of the abnormality [161].

\subsubsection{Computed Tomography (CT)}

The CT has mostly been replaced by the cranial MRI as the imaging modality of choice for brain tumors. Nonetheless it remains useful in selected situations [161]:

- Investigation of the skeleton and vascular system.

- Search for metastases in regions around the foramen magnum, the clivus or the skull base.

- In cases of emergency, where CT has a higher availability and a faster execution than MRI.

- In any cases where MRI is contraindicated (e.g. implants, containing iron).

\subsubsection{PET-Scans}

The Positron Emission Tomography (PET) is an excellent technique for the visualization of metabolic and functional processes. In the oncological context it is used to detect malignant tumors with high metabolic rates by the help of radiolabeled glucose and amino acid molecules [161]. Normally tumors exhibit an increased metabolic activity, which can be shown by a higher radiotracer influx 
compared to the surrounding tissues [161].

\subsubsection{Additional Imaging Methods}

Due to certain difficulties interpretation of MR images, which will be discussed in the following, and to improve the monitoring of tumors under treatment and during follow-up, there are certain methods, which can depict changes in the cellular metabolism (Magnetic resonance spectroscopy (MRS)), the perfusion (Perfusion$\mathrm{MRI}$ or $-\mathrm{CT}$ ), as well as the cellular texture of the tumor and perifocal edemas (Diffusion weighted imaging (DWI)) [151].

Also worth mentioning are the Functional MRI, to detect eloquent areas of the brain in the context of surgery, and the SPECT imaging (Single photon emission CT), which is used to detect abnormalities of the blood-brain barrier.

\subsubsection{Histopathology}

Histological examination of a putative brain tumor remains the most important component of the diagnostic evaluation of brain tumors. Furthermore, the microscopic examination is the precondition to differentiate between the various subtypes of neoplasms and to grade malignancy [28]. It is important to mention that astrocytomas extensively infiltrate into the healthy brain tissue, and especially the GBM proved to display a great variety in the extent, the geometry and the character of the infiltrating margin [28].

The WHO classification of 2007 comprises 4 different grades standing for an "ascending malignancy scale" of intracranial neoplasms. While grade I tumors are characterized by a low proliferative potential and offer the chance of cure after resection, neoplasms ranked in grad II show infiltrative growth and tend to recur. Moreover, some types of grade II tumors can progress to high-grade malignancies, for example the low-grade diffuse astrocytoma can develop to an anaplastic astrocytoma or even to GBM. The tumor classification of WHO grade III matches to histological evidence of malignancy such as strong mitotic activity or nuclear atypia $[93,157]$. 
Concluding, the WHO grade IV category refers to tumors characterized by microvascular proliferation and / or necrosis in addition to the aforementioned features $[93,157]$.

\subsubsection{Molecular Genetics}

The formation and progression of diffuse gliomas involve an accumulation of genetic alterations, which mainly affect the regulation of cell proliferation, differentiation, and apoptosis [18]. As with many other types of human cancers the activation of oncogenes, inactivation of tumor suppressor genes, abrogation of apoptotic genes and the deregulation of DNA repair genes play a critical role in the pathogenesis of gliomas [18].

As mentioned before, GBMs can arise de novo (primary GBM), usually in older patients, or evolve from low-grade gliomas (secondary GBM), normally in younger patients. The observed molecular changes differ in these two settings [18].

In the different steps of the evolution of malignant gliomas multiple pathways can be involved, which appear to include different molecular events. For instance, primary GBMs are associated with a variety of molecular changes, most remarkably the epidermal growth factor receptor (EGFR) amplification of overexpression [94]. Then again, in secondary glioblastomas and lower-grade gliomas, typically alterations of the IDH1 and IDH2 genes [165], a hypermethylation genotype [106] and inactivation of the TP53 tumor suppressor gene can be found [92]. In the changeover from low-grade to high-grad gliomas the inactivation of other tumor suppressor genes on chromosomes 9p, 13q and $19 q$ appear to be of significance $[143,166]$.

Since the survival of patients with malignant gliomas vary substantially and cannot be entirely explained by differences in histologic grading or clinical parameters, such as age or performance status, genetic markers may be valuable both, in assessing prognosis and response to treatment and to predict individual courses of disease [63, 67]. Today, genomic analysis can identify four different and 
clinically relevant subtypes of glioblastoma, which respond differently to treatment options as chemotherapy and radiotherapy, namely Proneural, Neural, Classical, and Mesenchymal. These four subtypes are characterized by abnormalities in the genes of PDGFRA, IDH1, EGFR and NF1 [147].

\subsection{Treatment and Prognosis of Malignant Gliomas}

\subsubsection{Initial surgical Approach}

The initial surgical resection is the treatment of choice for malignant gliomas. Although maximal resection is an important goal, the extent of surgery must be balanced with preservation of neurologic functions [17].

The tumor resection has three objectives [17]:

1. The extraction of sufficient tumor-tissue for accurate histological and molecular diagnostics.

2. The potentially improvement of clinical symptoms like seizures or neurological deficiencies.

3. The reduction of the tumorous cell population, in order to improve the overall prognosis.

Within a substantial retrospective study, including 416 patients, Lacroix et al. showed in 2001 that an aggressive resection of $98 \%$ or more of the tumor volume was a significant, independent predictor of survival in the entire patient population [84]. The best currently available data, concerning an influence of the extent of resection on the prognosis and the advantage of complete resection compared with partial resection, is given in a post-hoc-analysis of data from a prospectiverandomized study about fluorescence supported resection of glioblastoma [118]. This study could prove a significant advantage of total resection over partial resection regarding the median survival, which was 16,7 versus 11,8 months [111, $132,133]$.

In addition to the survival benefit, the surgical resection often comes along with an 
improvement of clinical symptoms [35]. Furthermore, an extended resection permits a more extensive extraction of sufficient tumorous tissue for histopathological diagnosis and molecular analysis, compared to stereotactic biopsy.

\subsubsection{Adjuvant Radiation Therapy}

Due to the high rate of recurrence in malignant gliomas, the adjuvant radiation therapy (RT) is indispensable, at least in malignant gliomas, as part of the standard-of-care therapy, using the so called involved field radiation therapy (IFRT), which has replaced the whole brain radiation therapy (WBRT) [27, 125]. Already in late 1970s the survival benefit of postoperative RT could be demonstrated in different studies and is still valid [11]. A daily application of 1,8-2,0 Gy up to a cumulative dose of 60 Gy in 6 weeks is the radiation-scheme which has shown the best results in GBM, as well as in grade III gliomas with a median survival of 9-12 months and 3-5 years, respectively [77].

MRI and functional imaging like $\left[{ }^{18} \mathrm{~F}\right]$ Fluorethyltyrosin(FET)-PET or MRS increasingly established themselves in the planning of radiation therapy [86].

\subsubsection{Adjuvant Chemotherapy}

Because of the blood-brain barrier, the applicability of cytostatic agents in the treatment of malignant brain tumors is extremely limited. The most utilized chemotherapeutics are the lomustine (CCNU) and nitrosoureas carmustine (BCNU), as well as the alkylating benzamid procarbazine [9].

Since 2005, the combination of RT and concomitant and adjuvant chemotherapy, using the alkylating agent temozolomide (TMZ), represents the standard regime in the treatment of WHO grad IV glioma after surgery [9].

In contrast to other combined chemotherapy and radiation regimes, which showed no significant survival benefit (e.g. RT plus mitomycin [59]), the concomitant and adjuvant TMZ application proved to result in a survival advantage [12, 134]. This 
statement was supported in a Cochrane review from 2008, published by Hart et al., conforming the therapeutic effectiveness and the low incidence of early adverse events caused by TMZ [62]. Recently, also Koukourakis et al. reviewed investigations on the mechanism of action as well as on resistance effects of TMZ[78]. Additionally, this review also presents current clinical studies and applications of temozolomide. As reported by Happold et al., patients with a methylated $0^{6}$-methyl-guanine DNA methyltransferase (MGMT) promoter show clinical benefits, being more sensitive to alkylating agents such as TMZ [60].

\subsubsection{Prognosis}

While the tumor grade is of critical prognostic value, also other criteria like the age of the patient, tumor location, proliferation indices, genetic alterations and many more are to be considered in the prediction of survival time [93]. In spite of substantial research for new therapeutic approaches the mean survival of a patient with malignant glioma could not be appreciably improved [93].

On average, patients who suffer from a WHO grade II glioma survive 5 to 7 years after diagnosis, while the ones who suffer from grade III gliomas have an average survival of 2-3 years. In contrast, patients with GBM (WHO grad IV) succumb to the disease in large part within 1 year after diagnosis [93, 116]. One of the rare improvements in the prognosis of GBM recently could be achieved by the concomitant and adjuvant application of TMZ plus RT, resulting in a prolongation of the survival period from 12 to 15 months [134]. So-called long-term survivors, who outlive the disease for more than 5 years, are very rarely seen $(3-5 \%$ of glioma patients) [80]. Exact reasons for such a long-term survival are still unclear, although several clinical factors seem to be beneficial, in particular young age at the point of diagnosis [80].

\subsection{Assessment of Response and Progression and its Limitations}

Despite aggressive therapy, in most cases glioblastoma eventually develop a progressive or recurrent course of disease. 
The individual decision making for each patient requires an assessment of both, response to treatment, as well as following diagnosis of a progressive disease.

Since 1990 the MacDonald-Criteria, which rely upon measurement of areas of contrast enhancement, have been the standard approach for patients with malignant glial tumors (Table I) [95]. Even if proved to be helpful these criteria are subject to a number of significant limitations, mainly due to innovative biologically active systemic therapy, as well as to substantial improvement in MRI technology [23]. The Response Assessment in Neuro-Oncology (RANO) Working Group, which is an international, multidisciplinary group, pointed out these limitations in 2010 and also proposed new approaches to evaluate the behavior of malignant gliomas [156, 158].

\subsubsection{MacDonald Criteria}

\subsubsection{Response Criteria}

The MacDonald Criteria for response assessment in high-malignant gliomas were primarily based on contrast-enhanced CT and the two-dimensional WHO oncology response criteria using enhancing tumor area (the product of the maximal crosssectional enhancing diameters) as the primary tumor measure [101]. In 2000 the Response Evaluation Criteria in Solid Tumors (RECIST) first introduced the use of one-dimensional measurements [138] and were recently revised (RECIST version 1.1 - [47]). Subsequently, different studies have compared the RECIST criteria with two-dimensional measurements, three dimensional measurements and volumetric measurements in malignant gliomas [53, 124, 152]. These studies show that there is a good conformance among the different methods in determining response in adult patients with both, newly diagnosed and recurrent high-grade gliomas [53, 124], as well as in pediatric brain tumors [152]. Still there have been no studies prospectively validating the RECIST criteria in gliomas. Therefore MacDonald Criteria are still widely used as a reference for assessing tumor response in clinical trials, but also for the reason that they enable the results of ongoing studies to be easily compared with historical data. 
The MacDonald Criteria define the following four possible outcomes:

Table I. Current Response Criteria for Malignant Gliomas

(Macdonald Criteria [95])

\begin{tabular}{ll}
\hline Response description & Response criteria \\
\hline Complete response (CR) & $\begin{array}{l}\text { Requires the following: } \\
\text { Disappearance of all enhancing measurable and } \\
\text { nonmeasurable tumor on consecutive CT or MRI scans } \\
\text { sustained for at least 1 month, no new lesions, no } \\
\text { corticosteroids, and neurologically stable or improved }\end{array}$ \\
\hline Partial response (PR) & $\begin{array}{l}\text { Requires all of the following: } \\
\geq 50 \% \text { reduction in cross-sectional area of all measurable } \\
\text { enhancing lesions on consecutive CT or MRI scans } \\
\text { sustained for at least 1 month, no new lesions, } \\
\text { corticosteroid stable or reduced, and neurologically stable } \\
\text { or improved }\end{array}$ \\
\hline Progressive disease (PD) & $\begin{array}{l}\text { Defined by any of the following: } \\
\geq 25 \% \text { increase in cross-sectional area of enhancing } \\
\text { lesions or any new lesion on CT or MRI scans, or } \\
\text { neurologically worsening and steroids stable or increased }\end{array}$ \\
\hline Stable disease (SD) & $\begin{array}{l}\text { Requires all of the following: } \\
\text { All other scenarios }\end{array}$ \\
\hline
\end{tabular}

\subsubsection{Limitations of the MacDonald Criteria}

Besides making new approaches in the evaluation of tumor response and progression the RANO Working Group pointed out the following limitations of MacDonald Criteria [158]:

- Pseudoprogression: Pseudoprogression is defined by increased contrast enhancement at the first reimaging after RT, which then resolves under continued therapy. Approximately one-fourth of patients with glioblastoma have evidence of pseudoprogression after receiving the standard treatment for malignant gliomas which consists of initial surgery followed by radiation therapy and concurrent and adjuvant chemotherapy (see 5.2.2 Imaging 
Phenomena: Pseudoprogression, Radiation Necrosis and Pseudoresponse).

- Surgical changes: Like Cairncross et al. already showed in 1985, a postoperative contrast enhancement in the wall of the surgical cavity in the first 48 to 72 hours is significantly common [29]. While neuroradiology is aiming to find a way to distinguish these areas from residual tumor, that failed to be resected, this confusion can easily be avoided by a baseline MRI obtained within 24 to 48 hours after surgery.

- Pseudoresponse under antiangiogenetic treatment: Recent approaches in glioblastoma therapy target the Vascular Endothelia Growth Factor (VEGF) pathway, which plays a critical role in the tumor genesis. First results showed an immediate decrease of contrast enhancement. Since the effect on overall survival is limited the conclusion of a tumor regression is doubtful. This effect, known as pseudoregression or pseudoresponse, proves another limitation of the MacDonald Criteria, namely that they do not take non-enhancing tumors into account (see 5.2.2 Imaging Phenomena: Pseudoprogression, Radiation Necrosis and Pseudoresponse).

- Other issues: Furthermore, irregularly shaped tumors, multiple tumors and enhancing lesions in cyst walls following surgery are not considered adequately.

Like Warmuth-Metz recently pointed out, the MacDonald Criteria also suffer from measuring inaccuracy, since they only rely on contrast enhancing tissue [151]. The enhancement of contrast can easily be influenced by changes in the amount of contrast applied, the contrast's kinetics or the MRI-sequence's sensitivity for the contrast agent. Also technical parameters like changes in sequences and the magnet-field's size can worsen that inaccuracy.

\subsubsection{The RANO Criteria}

The main complementing features contemplated by the RANO Working Group include the following criteria for progressive disease [158]: 
- Contrast enhancement outside the radiation therapy field or histologic evidence of viable tumor on biopsy sample during the first twelve weeks. The histologic analysis serves to distinguish pseudoprogression from real progression. Assuming progression only by clinical signs is not adequate during this initial period.

- New contrast enhancement outside the radiation field or an increase in size of the original lesion after the first 12 weeks. For patients taking an antianiogenetic drug such as bevacizumab the increase of nonenhancing lesion, as visualized in T2/T2 Flair image sequences, should be taken into consideration.

Concerning complete response, partial response, and stable disease the definitions of RANO Criteria confirm those from MacDonald.

Even if the RANO criteria take innovative treatment options and recent alterations in neuroimaging into account, they still lack in accuracy [24]:

- It is the first time that changes in T2-weighted sequences are taken into consideration, but there is no specific quantitative measurement given to evaluate these changes. This might be due to MR-parameters, as well as measurement technology, but to define progress as a "significant increase" of the lesion in T2/T2 Flair leaves much room for interpretation. However, up to date there are no reliable technologies existing to perform volumetric measurements.

\subsubsection{Conclusion}

As seen, despite extensive opportunities to make a relatively accurate diagnosis and even if the criteria for assessing response to treatment and progressive disease recently have been revised, this process obviously has much potential for improvements. To avoid that efficacious therapy will be discontinued prematurely, or that an ineffective treatment will be continued without benefit, it is very important to have an accurate and standardized way of assessing the response and 
progress in patients suffering from GBM. From the clinical perspective, a major issue is how to differentiate between pseudo-early progression and real early progression immediately after $\mathrm{RT}$.

\subsection{Objectives}

The aim of our study is to establish a new method of gaining tumor-specific information by analyzing MRI scans using DoctorEye - an open-source software tool. Our analysis will not only include the segmentation and volumetric measurement of tumor-suspicious and enhancing areas, as performed already, but also evaluate the distribution of signal intensities in forms of histograms. Additionally we will try to subdivide the pathologic findings into active tumor, necrosis and edema, to achieve more accurate results and to evaluate their development separately and in comparison.

Primary we want to find out, if our method reveals an average pattern of SIdistribution in newly diagnosed GBM. If so, we will proceed to review the patients follow up and to evaluate the SI changes during treatment, all with the goal of finding typical similarities, which can describe the tumors characteristics and might help to predict its development.

Additionally to the aim to generally improve the assessment of response to treatment and progressive disease, our study will consequently lead to the approach of differentiating real progression from pseudoprogression and radiation necrosis, real response from pseudoresponse and postoperative contrast enhancement from residual tumor. In addition our method might facilitate the essential differential-diagnosis of GBM to other intracranial lesions and the detection and measurement of multiple contrast-enhancing and irregular-shaped lesions. 


\section{Materials and Methods}

This research was done within the ContraCancrum- (Clinically Oriented Translational Cancer Multilevel Modelling; project reference number: 223979; http://contracancrum.eu/ ) and TUMOR-project(Transatlantic Tumour Model Repositories: project reference number: 247754; http://tumor-project.eu/ ), which were funded under the $7^{\text {th }}$ Framework Program of the European Commission.

Both projects and the related researches are approved by the Ethical Committee of the 'Ärztekammer des Saarlandes' at the $20^{\text {th }}$ of July 2010 (code number: 104/10; Appendix 9.4 Ethical Approval, Figure 38).

\subsection{Image Acquisition}

33 patients with assured or suspected glioblastoma were included into the study group. To maintain absolute confidentiality all 33 patients were anonymized as G0, $G 1, G 2,(\ldots)$, up to $G 32$. Except one, all of them were diagnosed within the years 2004 and 2008. The intracranial lesion from 4 of the 33 study subjects histologically turned out not to be a WHO Grade 4 glioma, whereas the intracranial lesion from 1 of the 33 study subjects could not be securely diagnosed (Table II). The corresponding MRI-files were provided by Prof. Reith, Head of the Department of Neuroradiology, at the University of Saarland, Homburg, Germany. In total this collection contained more than 22.000 single DICOM-images (Figure 1), including $T 1, T 1 \mathrm{Gd}-\mathrm{MRI}$ and $T 2$ sequences, which entirely have been examined and rendered.

Commonly used inclusion criteria as gender and age did not play a crucial role, since the statistical analysis of the pathologic MRI-scans was the study's key aspect (Figure 2).

Finally, only scans created at the time of diagnosis, after surgery (if performed) and after RT and chemotherapy were taken into account, to provide a 
standardized overview of the patient's individual course of disease and an easy comparability between the study subjects.

Due to the individuality of the different cases and the aggressiveness of the disease, a complete follow up (MRI-scans at diagnosis, after surgery, after radioand chemotherapy) was available in 9 of the 33 cases.

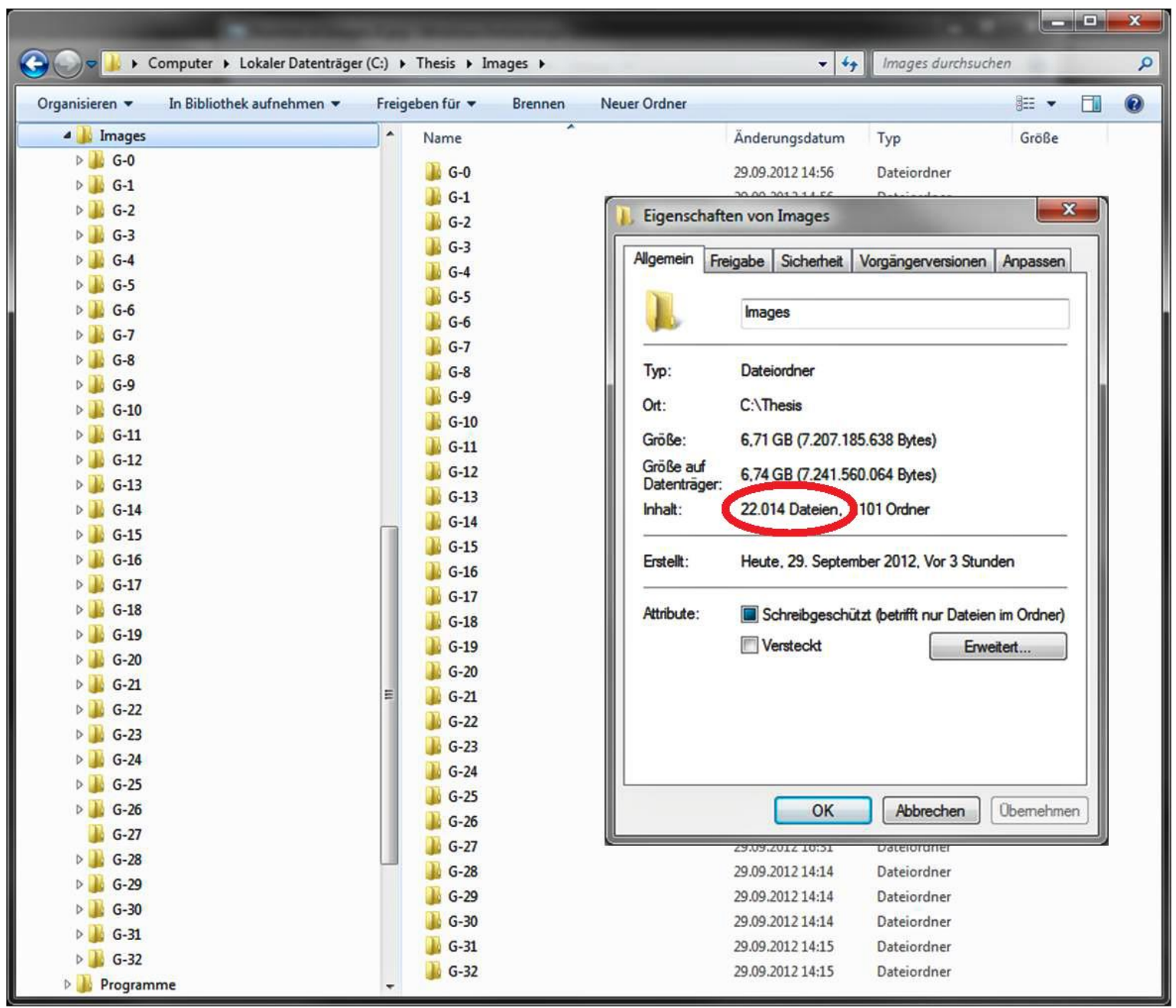

Figure 1: DICOM Data-Set

DICOM data-set of whole study group (33 patients), with 22.014 files and a total size of 6,71 Gigabytes. 
Table II. Study Group - Date of Diagnosis, Histology and WHO grading

\begin{tabular}{|c|c|c|c|}
\hline Pseudonym & Date of diagnosis & Histology & WHO grading \\
\hline G-0 & 11.11 .2005 & Astrocytoma & 2 \\
\hline G-1 & 22.01 .2008 & Anaplastic Carcinoma & - \\
\hline G-2 & 15.02 .2008 & Glioblastoma & 4 \\
\hline G-3 & 12.09 .2007 & Glioblastoma & 4 \\
\hline G-4 & 09.01 .2008 & Glioblastoma & 4 \\
\hline G-5 & 29.06 .2007 & Glioblastoma & 4 \\
\hline G-6 & 27.10 .2006 & Glioblastoma & 4 \\
\hline G-7 & 11.02 .2008 & Glioblastoma & 4 \\
\hline G-8 & 15.02 .2008 & Bronchial Cancer & - \\
\hline G-9 & 18.02 .2008 & Glioblastoma & 4 \\
\hline G-10 & 19.02 .2008 & Glioblastoma & 4 \\
\hline G-11 & 25.02 .2008 & Glioblastoma & 4 \\
\hline G-12 & 26.02 .2008 & Glioblastoma & 4 \\
\hline G-13 & 05.03 .2008 & Glioblastoma & 4 \\
\hline G-14 & 08.04 .2008 & Glioblastoma & 4 \\
\hline G-15 & 14.04 .2008 & Astrocytoma & 3 \\
\hline G-16 & 22.04 .2008 & Glioblastoma & 4 \\
\hline G-17 & 15.05 .2008 & Glioblastoma & 4 \\
\hline G-18 & 20.06 .2008 & Glioblastoma & 4 \\
\hline G-19 & 23.07 .2008 & Glioblastoma & 4 \\
\hline G-20 & 30.07 .2008 & Glioblastoma & 4 \\
\hline G-21 & 04.08 .2008 & Glioblastoma & 4 \\
\hline G-22 & 19.08 .2008 & Glioblastoma & 4 \\
\hline G-23 & 26.03 .2008 & Glioblastoma & 4 \\
\hline G-24 & 04.09 .2008 & Glioblastoma & 4 \\
\hline G-25 & 19.09 .2008 & Glioblastoma & 4 \\
\hline G-26 & 26.11 .1998 & Glioblastoma & 4 \\
\hline G-27 & 24.08 .2004 & Glioblastoma & 4 \\
\hline G-28 & 22.03 .2006 & & - \\
\hline G-29 & 02.05 .2006 & Glioblastoma & 4 \\
\hline G-30 & 05.05 .2006 & Glioblastoma & 4 \\
\hline G-31 & 25.04 .2005 & Glioblastoma & 4 \\
\hline G-32 & 09.03 .2006 & Glioblastoma & 4 \\
\hline & & & \\
\hline & & & 4 \\
\hline
\end{tabular}




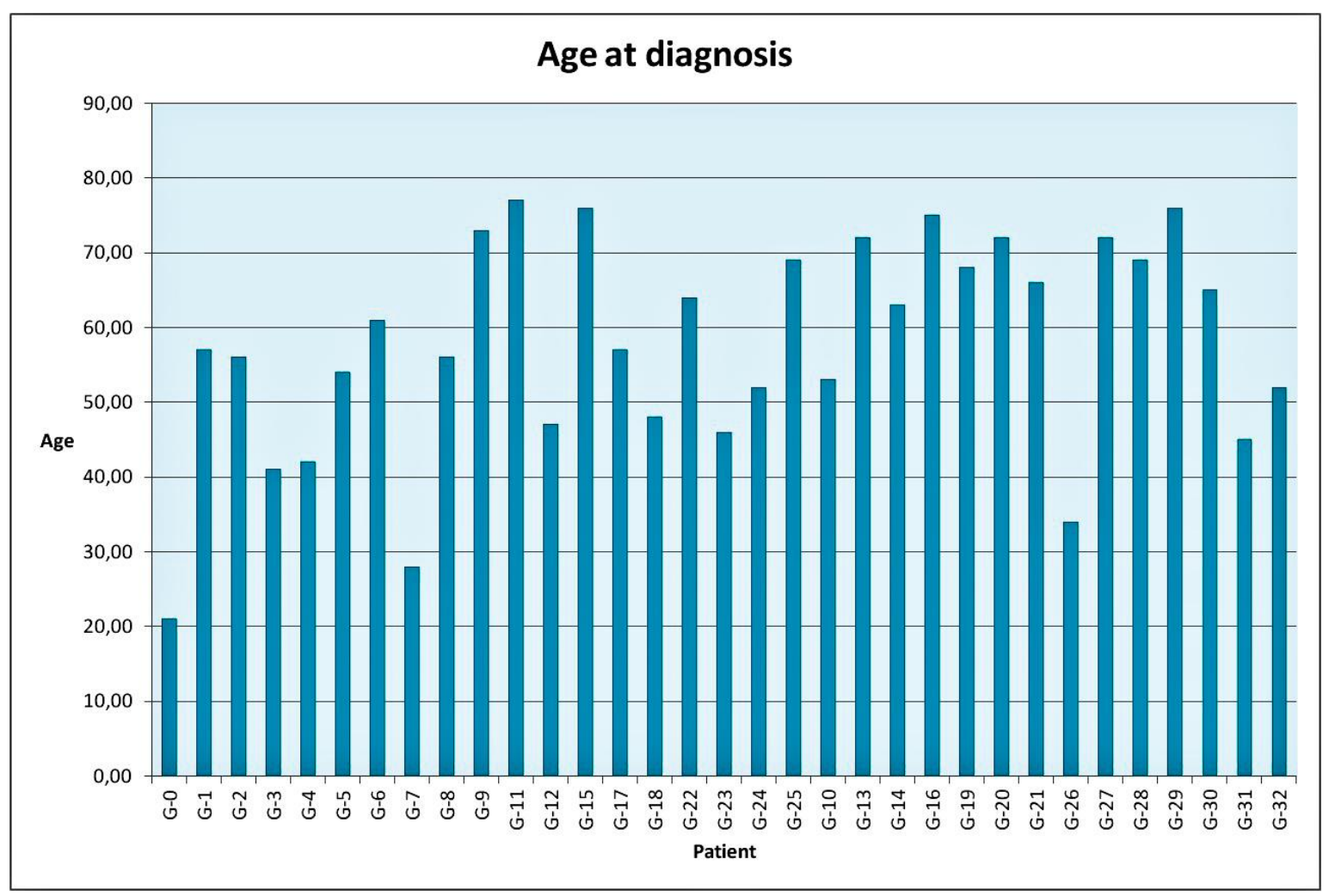

Figure 2: Age Distribution of the Study Group

\subsection{Investigation Process and Equipment Technology}

\subsubsection{Principles of the MRI}

The magnetic resonance imaging is a medical imaging technique, which is based on the physical principles of the nuclear magnetic resonance (NMR). Bloch and Purcell in 1946 coincidently developed a method to detect this nuclear resonance [13]. In 2003 Paul Lauterbur and Sir Peter Mansfield even were awarded with the Nobel Prize in Physiology or Medicine for their discoveries concerning the field of MRI [13].

An MRI scanner is a device that is able to generate a strong static magnetic field. Atomic nuclei have an intrinsic angular momentum (nuclear spin), which describes the direction and strength of a magnetic field surrounding the nucleus and is substance-specific [38]. The most important element for NMR investigations is the 
nucleus of hydrogen, the proton, which is the most basic and the most frequent atomic nucleus in the human body [13].

While the patient lies within the scanner, the imaging magnet produces a strong, constant external magnetic field. Under the influence of this magnetic field a fractional part of the nuclei get directed into the direction of the static field, thus generating a macroscopic, measurable magnetic moment [38]. The induced motion of the spin around the magnetic field is called precession and is proportional to the strength of the applied field. The rate at which the spin precesses is known as the Larmor frequency [13].

\subsubsection{Image Generation}

To generate an image, the protons have to be excited. This excitation is achieved by the application of an additional, momentary, high-frequency alternating field. Immediately after their excitation, the nuclei return to their ground state, a measurable process that is called relaxation, which differs depending on the chemical compound, and the molecular environment of the hydrogen atoms, whereby different signal intensities are generated in the resulting image [117].

The relaxation is composed of two different processes, the longitudinal and transversal relaxations, which are described through tissue-specific time constants, T1 for the longitudinal relaxation, lasting $300-2000 \mathrm{msec}$, and T2 for the transversal relaxation, lasting $30-150 \mathrm{msec}$. Since the impulse that is emitted during the longitudinal relaxation is very little, protons are excited repeatedly by a so called pulse-sequence. The repetition time (TR) is the amount of time between two single pulses, whereas the elapsed time from the pulse to the peak of the echo is called the echo time (TE) [117].

Different body tissues generate different signal intensities in MR images, depending on the specific tissue properties ( $T 1, T 1$, and proton density), sequence parameters (TR and TE) and the type of sequence. Thus, a contrast between different tissues can be generated [117]. 


\subsubsection{MRI Sequences}

The MRI includes a broad range of imaging sequences, however, our investigations are limited to only a part of these. In our study we examined the two classical sequences T1 and T2, as well as their variations, T1 Gd-MRI and T2 Flair. Other sequences that are comprised within the spectrum of MRI, although not part of our study, include susceptibility-weighted imaging (SWI), diffusion- and perfusion-weighted imaging (DWI and PWI), and MR angiography.

The T1-and T2-weighted images are the classical sequences for the anatomical representation of the brain and the CSF. The T1-weighted imaging refers to the longitudinal relaxation and describes the process of the regeneration of the longitudinal magnetization, dissipating measurable energy (spin-lattice relaxation). It is characterized by a short TE and TR. Typically tissues that have a short T1 time constant and contain lipids are displayed hyperintense (e.g. white matter and fat), whereas tissues with a long T1 time constant appear hypointense (e.g. gray matter, muscle and CSF) $[13,117]$. The T2-relaxation time, referring to the transversal relaxation, is determined by the rate of the reduction of the transverse magnetization, a process that does not emit energy, and can draw conclusions about the interaction of the spins between various nuclei (spin-spin relaxation). The T2-sequence has typically longer TE and TR and displays tissues with a long T2 time constant hyperintense (e.g. water), whereas tissues with a short T2 time constant appear hypointense on T2-weighted images (e.g. muscle) $[13,117]$.

Usually, a clinical MRI examination includes T1-and T2-weighted image series in at least two spatial planes. In the gadolinium contrast-based T1-weighted sequence paramagnetic contrast agents (chelates of gadolinium) are used and administered intravenously. On such a T1-weighted image the accumulation of the contrast-agent is represented by increased $\mathrm{SI}$, and is usually equivalent to an increased vascularity [38]. In the T2 Flair sequence the very high signals from the CSF in T2-weighted images are suppressed, which is of particular benefit if one wants to define a lesion that adjoins to the CSF [13]. 
By the variation of pulse sequences the MRI is able to emphasize on differences in T1, T2 or proton density. Based on the choice of the imaging sequence it is possible to detect small differences in the microenvironment of different tissues, being flexible in determining the tissue contrast [38].

\subsubsection{MRI Scanner}

In all patients cranial MR images were acquired by the use of a 1.5 Tesla wholebody MRI scanner produced by Siemens (Siemens 1.5T MAGNETOM Vision; Figure 3).

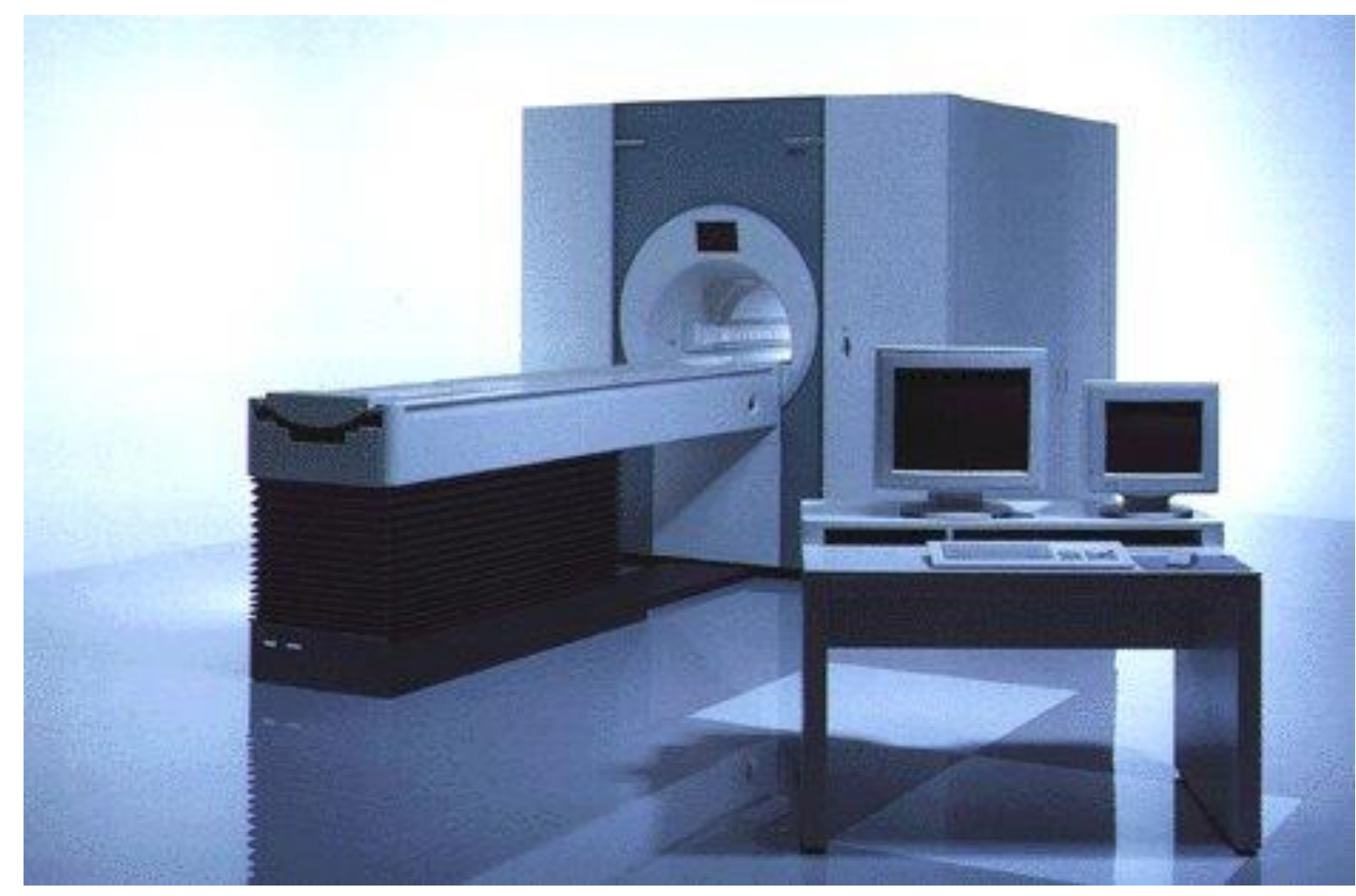

Figure 3: Siemens 1.5T MAGNETOM Vision MRI Scanner [8]

\subsection{Image-Analysis}

All the images have been preprocessed and analyzed at the Department of Pediatric Oncology and Hematology from the University of the Saarland between the years 2010 and 2012. The image-rendering was performed with the DoctorEye software, a multifunctional open platform for fast annotation and visualization of 
tumors in medical images [128], while the statistical analysis was done using Microsoft Office Excel 2007.

\subsubsection{Background on DoctorEye (4.7)}

DoctorEye is a flexible and easy-to-use annotation platform with a Graphical User Interface (GUI) for quick and precise identification and delineation of tumors in medical images, under the GNU General Public License [128]. Being an opensource software tool, DoctorEye easily can be downloaded and tested (http://biomodeling.ics.forth.gr; Figure 4). As a clinically driven, interactive and multifunctional platform, DoctorEye is frequently revised, to ensure that it can be efficiently and intuitively used to annotate large numbers of tomographic datasets in the clinical setting. Our close collaboration during the research with the developers from the biomodeling group of the BioMedical Informatics [BMI] laboratory of FORTH ${ }^{1}$ in designing the platform helped to optimize the software's user-friendliness and its practical applicability.

1 Foundation for Research and Technology - Hellas, Crete, Greece; http://www.forth.gr/ 


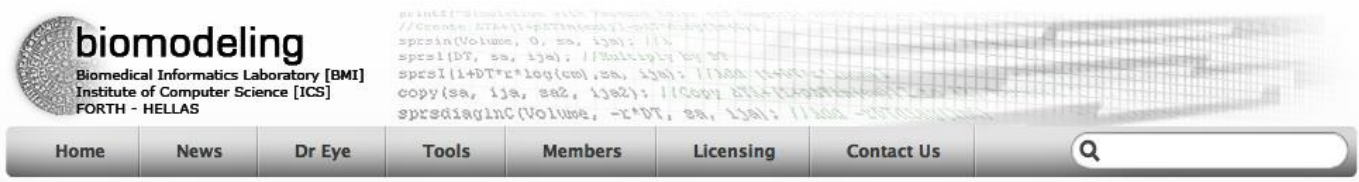

\section{Dr Eye}

Dr Eye is a flexible and easy-to-use annotation platform (GUI) for quick and precise identification and delineation of tumors in medical images.

The design of the platform is clinically driven in order to ensure that the clinician can efficiently and intuitively annotate large number of 3D tomographic datasets.

Both manual and well-known semi-automatic segmentation techniques are available in the platform allowing clinician to annotate multiple regions of interest at the same session. Additionally, it includes contour drawing, refinement and labeling tools that can effectively assist in the delineation of tumors. Furthermore, segmented tumor regions can be annotated, labeled, deleted, added and redefined. The platform has been tested over several MRI datasets to assess usability, extensibility and robustness with promising results.

\section{Licensing}

Dr Eye's licensing

\section{Download}

Download Prerequisite. NET Framework 3.5 from here. Download Dr Eye manual from here.

\section{Plugins}

In this section you can find plugins developed by 3rd party developers. Right now you can visit the website of UniBe, which has developed a plugin suite called "Doctor-No" and which includes 4 tools for skull stripping, isotropic resampling, registration and tissue classification. The plugins \& a manual can be downloaded from here.

\section{For Developers}

Download the library files and the documentation needed to create plugins for the $\mathrm{Dr}$ Eye platform.

Download the SDK basic examples in order to create plugins for the Dr Eye platform.

\section{B M I}

Contra *ancrum Cancer Multileve

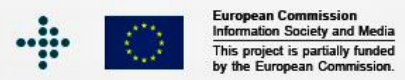

DrEye_ver4.7.0

Click to download the latest version of Dr Eye
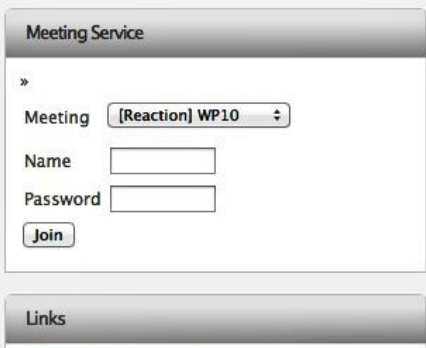

» BioMedical Informatics Laboratory

" Contra Cancrum

" European Commission

" Foundation for Research and Technology -

" Foundation for

Archives

»April 2010 (1)

\section{Figure 4: DoctorEye Webpage}

Publicly accessible webpage of the biomodeling group, which is part of the BioMedical Informations [BMI] laboratory, of FORTH, offering free download of the DoctorEye software and associated files - http://biomodeling.ics.forth.gr. 


\subsubsection{Selected Segmentation Method - The Magic Wand Algorithm}

The "Magic Wand" Algorithm is a segmentation tool for semiautomatic rendering, which provides high accuracy. Its function is based on finding and selecting all the pixels neighboring a predefined, user-selected initial point that are sufficiently similar in gray level. To determine how broad the range of summarized gray levels is, a tolerance value can also be set by the user (higher tolerance ends up in larger selection). If the desired preciseness cannot be achieved by only using the magic wand, DoctorEye offers two additional tools for manual refinement, the "Pencil" and the "Eraser" (Figure 5).

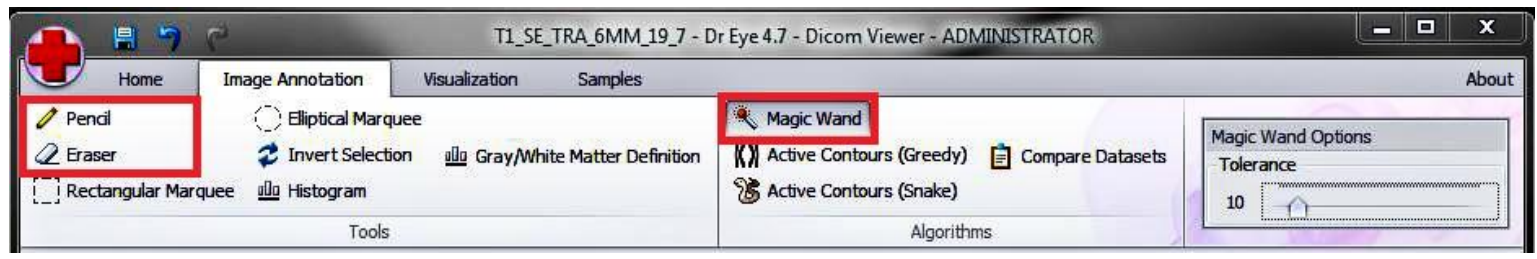

Figure 5: DoctorEye Task Bar with the Annotation-Tools "Pencil, Eraser" and the "Magic Wand"

\subsubsection{Preprocessing}

Segmentations were performed of sequences T1, T1 Gd-MRI, T2 and T2 Flair. To specify our results suspected active tumor tissue, necrosis, edema and the cerebrospinal fluid (CSF) were separately analyzed in all mentioned sequences. To ensure the highest accuracy, all the segmentations were performed by semiautomatic rendering of the areas of interest using the so-called "Magic Wand Algorithm". Figure 6 shows an exemplary graphical user interface of the DoctorEye 4.7 software, while Figure 7 illustrates the comparison between the raw and the processed version of an MR-Image. Additionally, DoctorEye offers the option to create a three-dimensional visualization of the segmented tumor including all annotations.

Signal intensities of the CSF were used as reference values for standardization of signal intensities within the tumor. After rendering tumor volumes were calculated. To guarantee the highest grade of correctness supervision was performed by Prof. 
Dr. Reith, an expert in the field of neuroradiology and Head of the Department of Neuroradiology at the University of Saarland, Homburg, Germany.

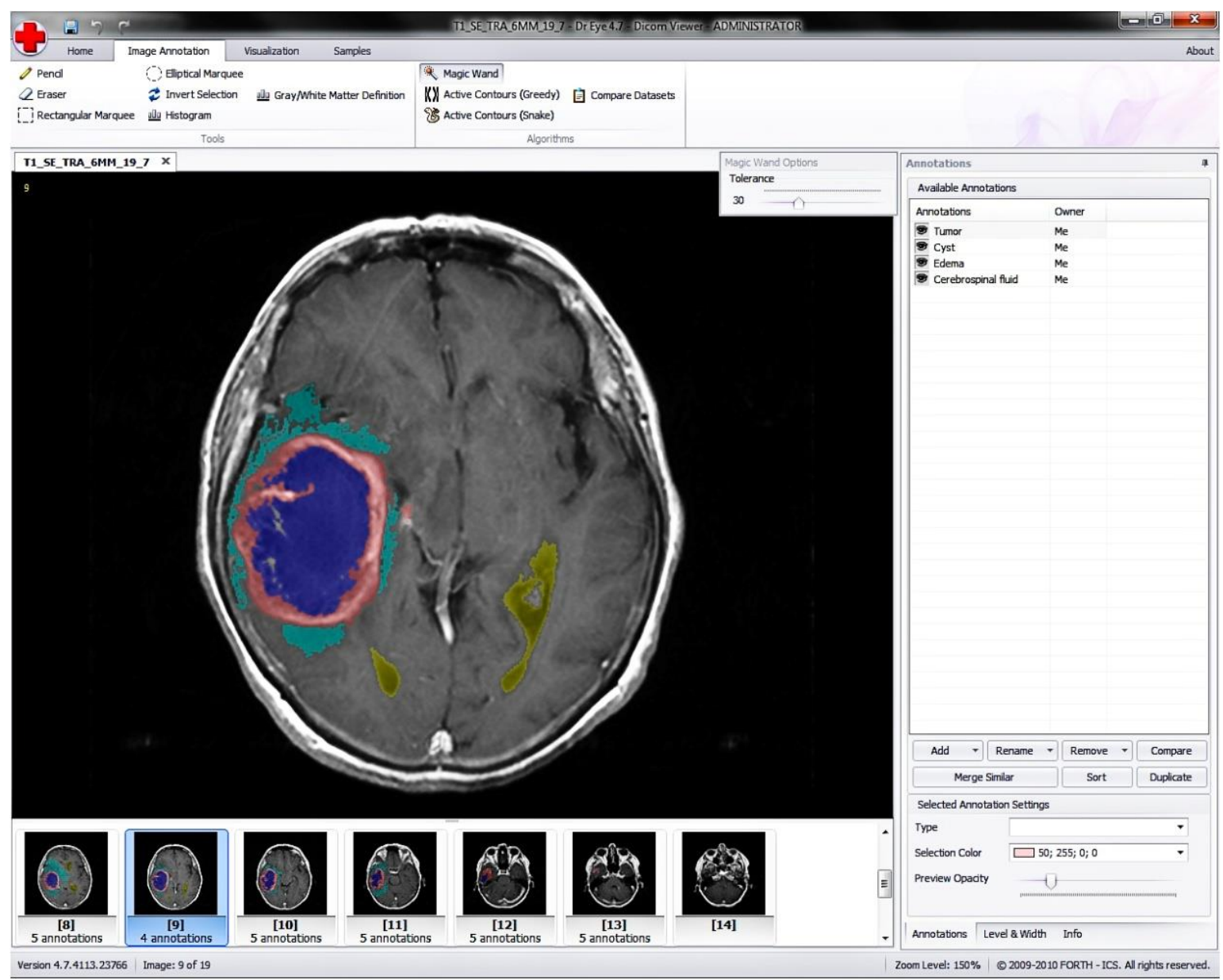

Figure 6: Graphical User Interface (GUI) of DoctorEye 4.7.

This snapshot of the software's main window shows an example of a glioblastoma at the time of diagnosis. The contrast enhanced tumor is shown in red, cystic/necrotic area in dark blue/purple and edematous area in light blue. Cerebrospinal fluid is marked in yellow (only part of the left and right side ventricle). 


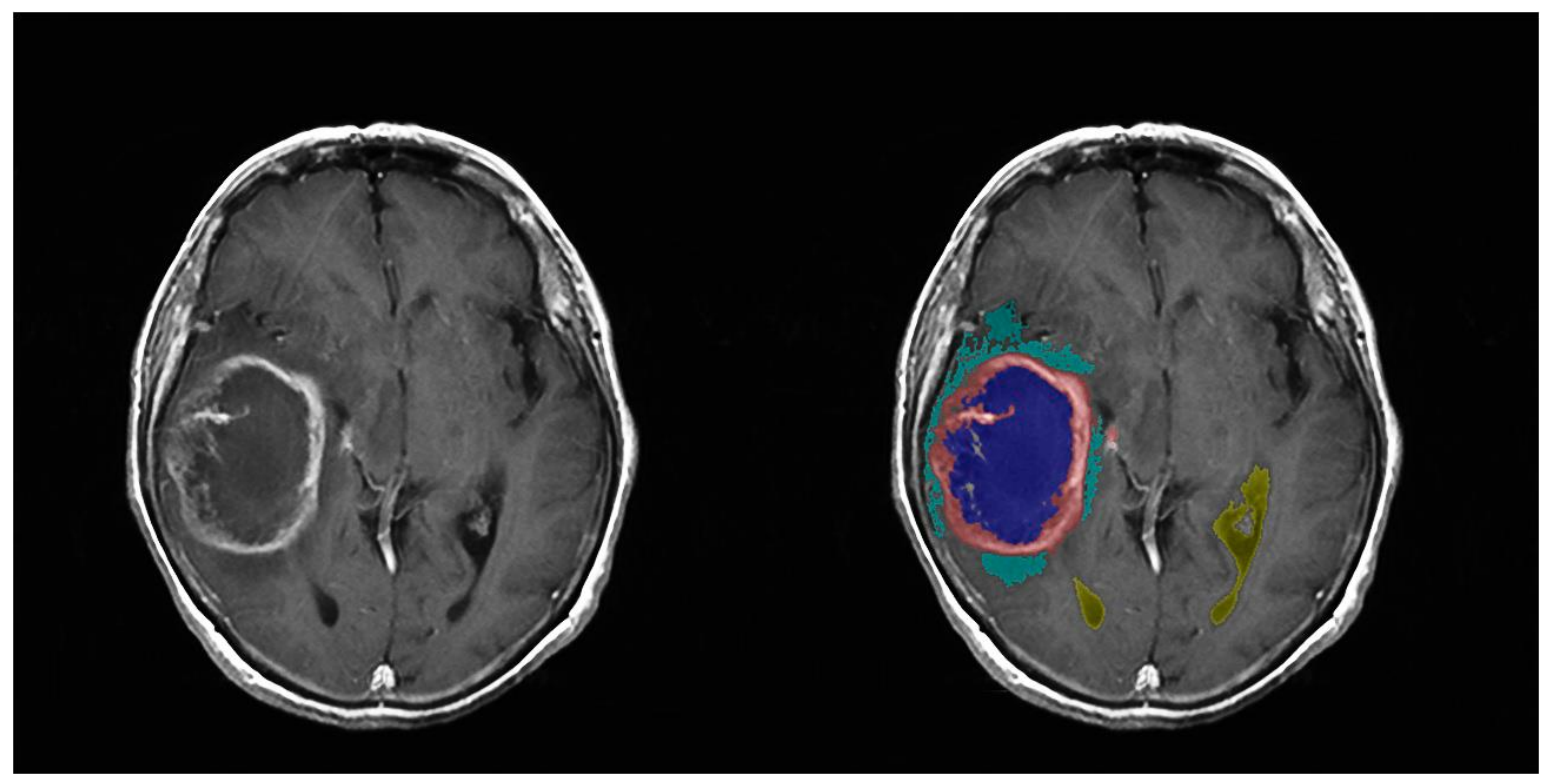

Figure 7: Comparison between raw and processed MR-Image

For illustrative purpose, the comparison between a raw (left side) and a processed MR-Image (right side) from Patient G-13 at diagnosis is shown (sequence $=T 1$ with gadolinium contrast). The contrast enhanced tumor is shown in red, the cystic/necrotic area in dark blue/purple and the edematous area in light blue. Cerebrospinal fluid is marked in yellow (only parts of the left and right side ventricle).

\subsubsection{Measurements and Analysis}

DoctorEye provides the calculation of histograms of the rendered areas and the corresponding mean and median values (Figure 8). Histograms were created in every patient, based on the four different areas of interest (active tumor, necrosis, edema and CSF), in all four investigated MRI-sequences ( $\mathrm{T} 1$ without contrast, $\mathrm{T} 1$ Gd-MRI, T2 and T2 Flair). The histograms' data were uploaded to Microsoft Office Excel 2007, to perform further statistics, including the normalization of the data (Figure 9; 3.3.5 Statistical Analysis). A comparisons of the histograms' graphical shape and the signal intensities' mean and median value of different tumor areas were performed for each sequence, at the time of diagnosis and during follow-up, in individual patients and inter-individually. In 9 of the 33 patients a complete follow-up could be analyzed, referring to DICOM data-sets at diagnosis, after surgery and after radio- and chemotherapy. Up to now, a quantitative analysis of signal intensities is not possible, due to the variety of MR machines and the lack of standardization in producing MR-images. 


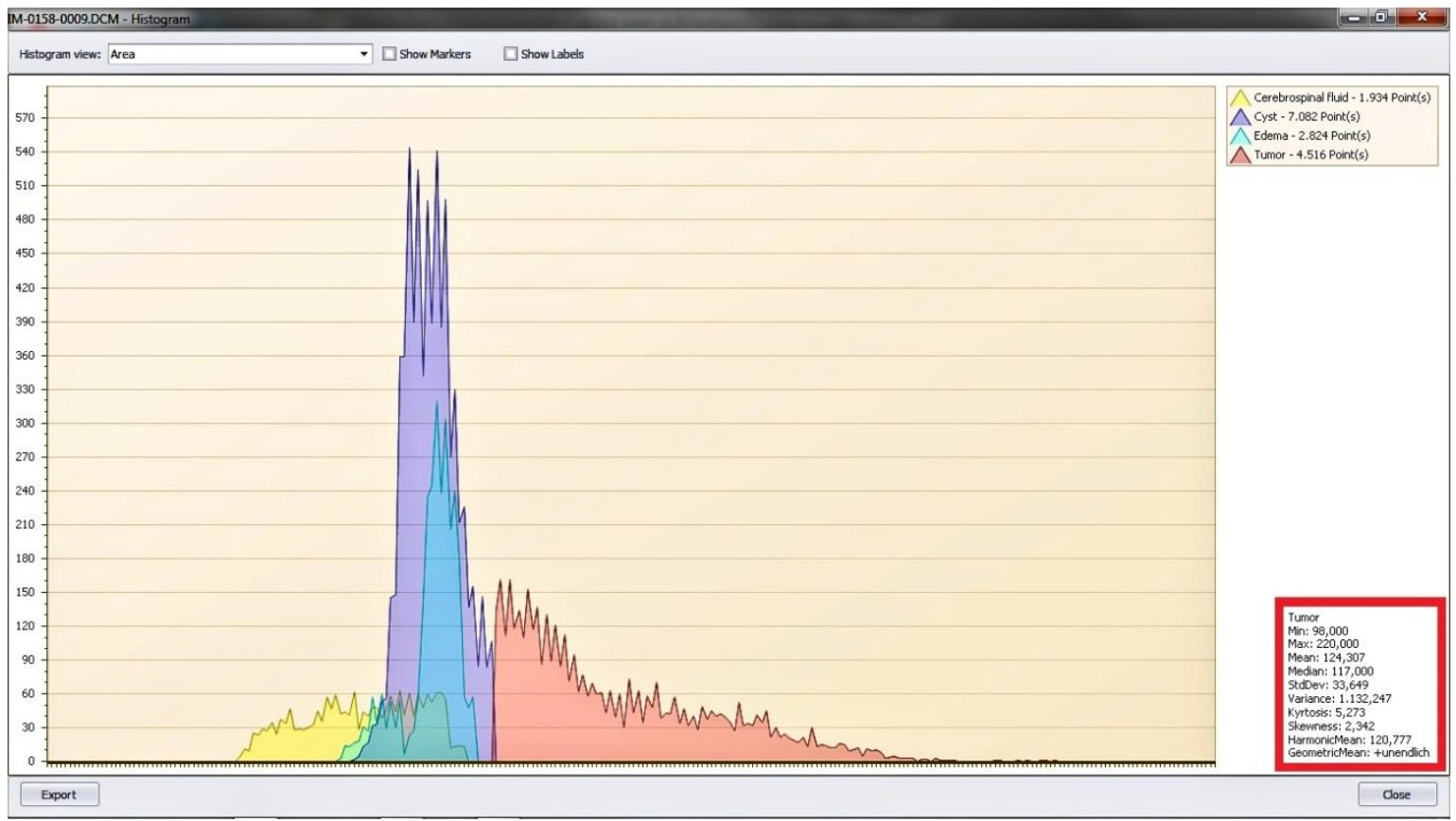

Figure 8: Histogram calculated by DoctorEye

This diagram shows the graphical representation of a signal-intensity histogram, automatically provided by the DoctorEye software. The histogram corresponds to the rendered MR-Image in Figure 6 and Figure 7.

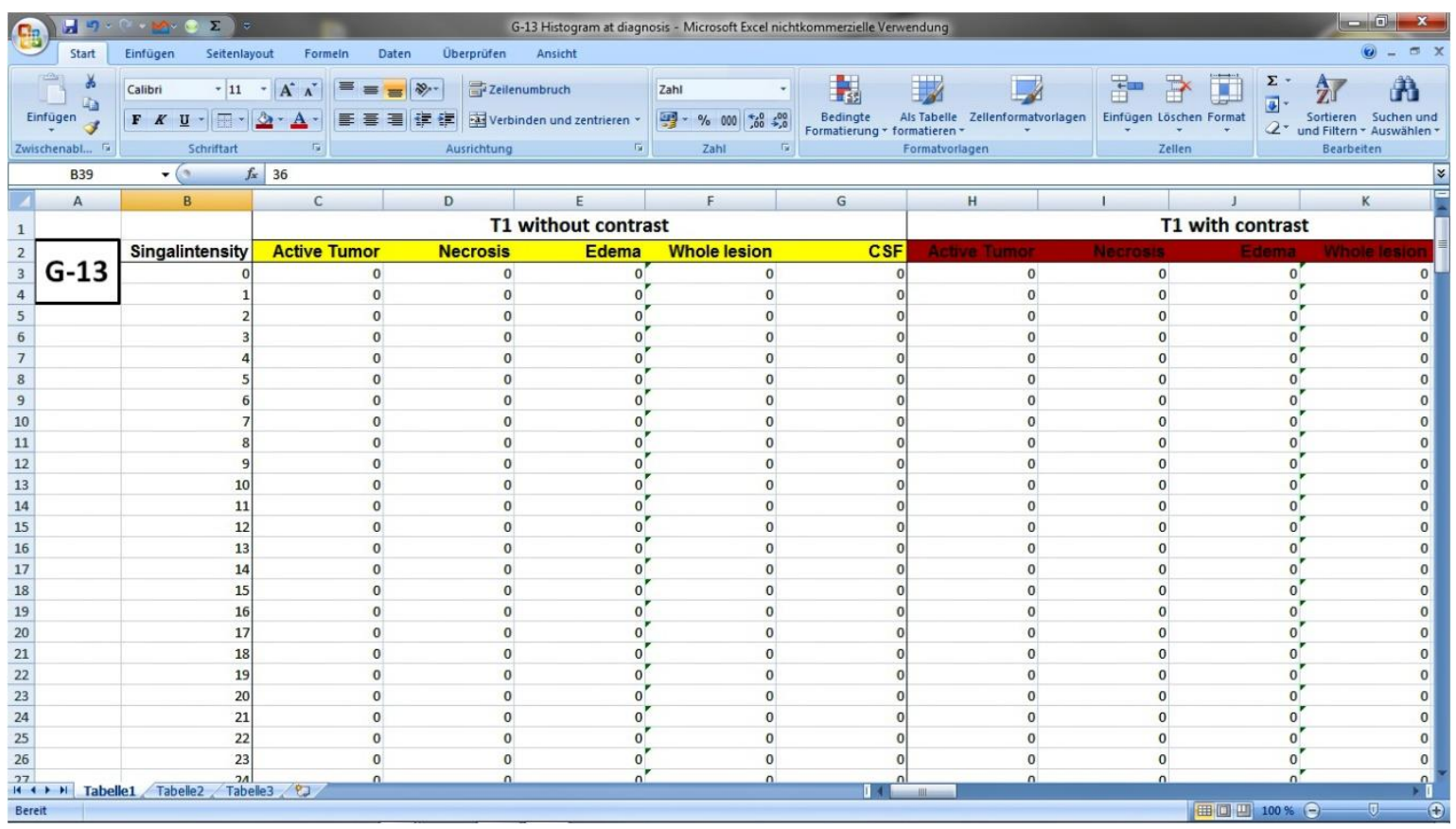

Figure 9: Histogram Data Transfer into Microsoft Office Excel 2007 


\subsubsection{Statistical Analysis}

The definition of possible outcomes in our study for the evaluation of our results from active tumor, necrosis and edema and their volume-development was based on the RECIST criteria, which were published in 2000 [138]. The definition of response requires a decrease of tumor size of more than $50 \%$, whereas progressive disease equals an increase of more than $25 \%$. Stable disease is a status in which neither the criteria for response nor for progressive disease are complied. The two exceptionally conditions pseudoprogression and pseudoresponse have been described inter alia in the RANO criteria from 2010 [158]. Since in our patient collective no antiangiogenetic drugs were administered, we termed the status in which the development of the whole lesion indicates response to treatment, while at the same time the remaining results are contradictory, "questionable response" instead of pseudoresponse.

The statistical analysis of the histograms, provided by DoctorEye, based on our segmentations, followed the subsequent steps and was performed in every single histogram created:

1. Transfers of the data into a Microsoft Office Excel 2007 spreadsheet, creating for each MRI sequence four excel columns corresponding to the different segmentations, namely "active tumor, necrosis, edema" and "CSF".

2. Summarization of the histograms of "active tumor, necrosis" and "edema" to an additional excel histogram called "whole lesion".

3. Calculation of the total pixel count for each histogram.

4. Calculation of the annotation's volume for each histogram by multiplying the total pixel count by the volume of a single pixel corresponding to the specific image set.

5. Calculation of SI-peak for each histogram.

6. Calculation of mean SI:

Sum of all SI-values multiplied with corresponding pixel-count, divided by sum of all pixels in that specific histogram. 
7. Standardization of mean SI:

Mean SI of the three different tumor-qualities (active tumor, necrosis, and edema) divided by mean SI of the CSF.

Thereafter the histograms of the whole study group were summarized by calculating an average histogram for each of the four areas of interest (active tumor, necrosis, edema and CSF), in all MRI-sequences investigated. The resulting mean histograms underwent the same steps mentioned before in 1.-7., calculating an average- total pixel count, tumor-volume, SI-peak, mean SI and standardized mean SI. An exemplary screenshot of such a calculation spreadsheet is given in Figure $\mathbf{1 0}$. 


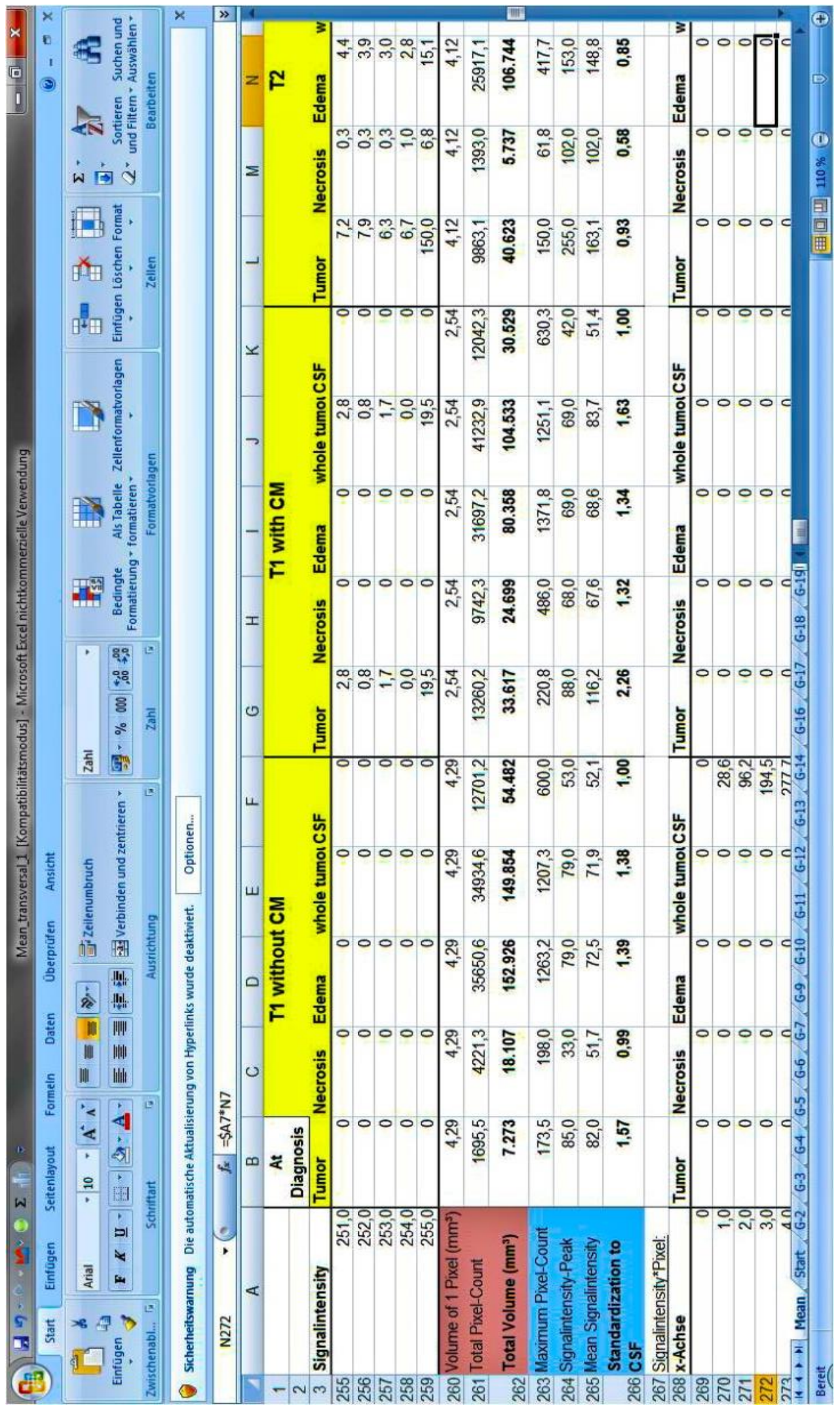

Figure 10: Histogram Analysis in Microsoft Office Word 2007 


\section{Results}

\subsection{Evaluation of DoctorEye}

DoctorEye presented as an user-friendly segmentation software which allowed us to perform fast annotations of different data sets at the same time, with a high level of accuracy.

During the two year research we stood in a lively exchange with the developers of FORTH. Our suggestions for improvement had a great influence on the software updates, particularly in optimizing the practical handling and its applicability in the clinical daily routine.

\subsubsection{Processing Time}

DoctorEye protrudes with its fast reaction and short processing time, which permits a smooth and steady workflow. For the following mentioned procedure, we used a laptop with "Windows 7 Professional", an Intel ${ }^{\circledR}$ Core ${ }^{\mathrm{T}} 2$ Duo processor at $2,20 \mathrm{GHz}$, and $4 \mathrm{~GB}$ of memory (RAM). The time that is required for an unpracticed user to completely process a directory of DICOM files, using mainly the "Magic Wand" algorithm, and to export the acquired histograms is listed below:

- For getting the program started and ready-to-use: less than 15 seconds.

- For uploading and opening one set of Images, for example T1 Gd-MRI, containing 19 slices of already annotated images and a file size of 10,2 MB (Patient $=\mathrm{G}-13$; at diagnosis): less than 10 seconds.

- For the investigation, differentiation between active tumor, necrosis, edema and CSF and their semiautomatic rendering in the given example (G-13 at diagnosis), whose lesion is rather voluminous and extends over 9 of the 19 slices: 16:54 minutes.

- For the processing of series of average complexity, in comparable size to the above mentioned: approximately 15 minutes.

- For assembling the corresponding histograms: 3 seconds 
- For saving the processed images, respectively the completed annotations: 2-3 seconds.

- For exporting the histogram-data in Excel file format: 30 seconds.

Naturally an experienced person could distinguish and annotate the lesions in a much faster way. The same applies if the semiautomatic segmentation method is replaced by a less time-consuming automatic or semi-automatic method.

To facilitate the usage of the software, all important features have their own keyboard short-cut.

\subsubsection{Accuracy}

If very high accuracy is required the semiautomatic segmentation method "magic wand" is the method of choice, even if it goes at the expense of the processing time.

Beside technical properties, the user's knowledge in distinguishing between different cerebral tissues is of great importance in that specific context. In our case training, control and confirmation was performed by Prof. Reith, Head of the Department of Neuroradiology, at the University of Saarland, Homburg, Germany. Even though the supervision kept on regularly during the whole research period the results already have been promising after a few hours of practice.

As Figure 11 and 12 impressively illustrate, DoctorEye enables the user to perform most precise delineation between different areas of interest. The combination of the tools "Magic Wand, Pencil and Eraser" makes it possible to correct small details while still being effective in terms of time management. In every session the user is able to set a personal balance between accuracy and processing time, depending on current priorities in the clinical setting. If certain areas are difficult to demarcate, due to their structural complexity, it is possible to change from the "Stretch" mode, which makes the current image fill as much of the screen as possible, to the "Zoom In" mode. By using this feature, it is possible to magnify the selected area of the image to different degrees and thereby to 
facilitate the differentiation and segmentation of the investigated structures. Holding the shift-key allows the user to include areas to the currently selected annotation, which are not in direct contact to the initial point or included in the preset tolerance value. This is very helpful when one wants to partially enlarge the marked area without increasing the tolerance level. Furthermore, the "UndoFunction" can be used if the last added annotation not conforms to what was the desired area, while it is not wished to delete all previously selected parts of this annotation. This makes it possible to retry the selection with an unlimited number of attempts, until the result is satisfying.
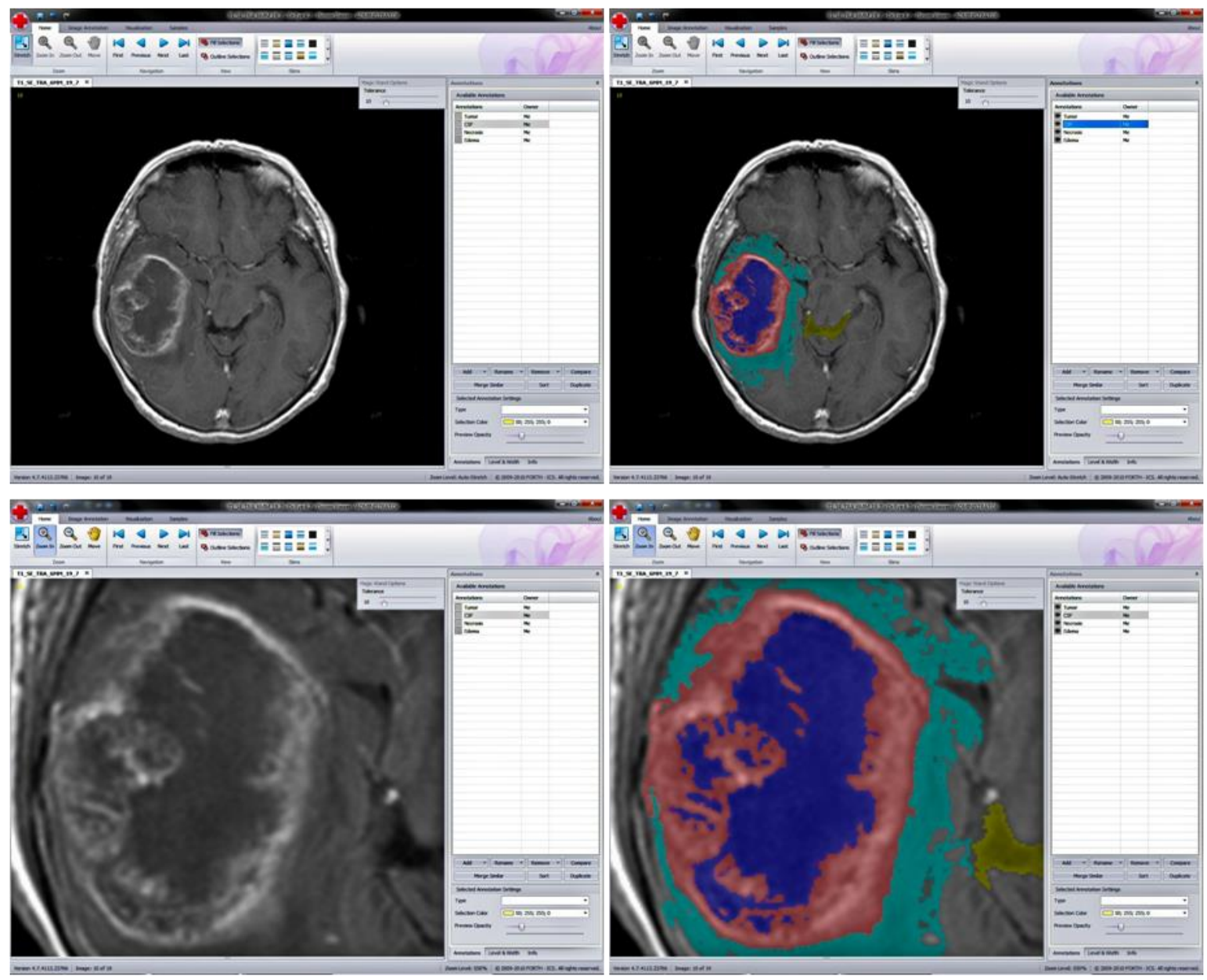

Figure 11: Illustration of DoctorEye's high Potential of precise Delineation

Left side: raw images, right side: processed images.

Upper row: image overview, lower row: amplified image

For easier delineation and to achieve more precise segmentations of the areas of interest DoctorEye allows the user to "zoom in" on the image using the "Magic Wand" algorithm for segmentation and the "Pencil and Eraser" tool for manual refinement. 


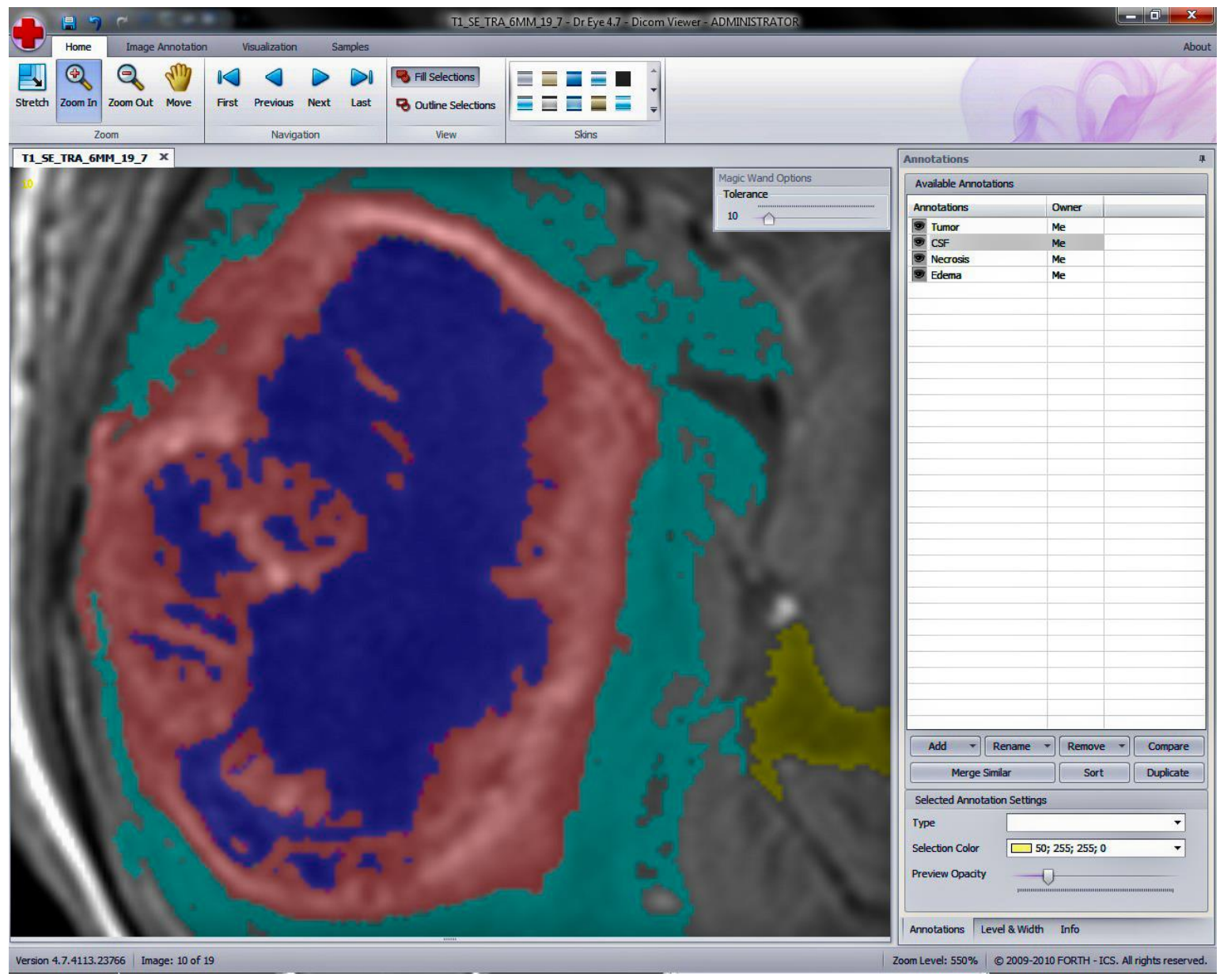

Figure 12: Illustration of the Software's high grade Accuracy in an amplified Image

\subsubsection{D-Visualization}

Additionally to segmentation- and histogram-preparation, DoctorEye offers the option to create a three-dimensional visualization of the segmented tumor and all other findings. Figure 13 demonstrates this feature, which is given in an interactive window that provides the user with a choice of different environment settings and the possibility to change parameters concerning the viewing angle, the color of the annotations and many more, for the best spatial representation of the annotations.

The illustrated example is the result of the segmentation of 19 cranial MR images (T1 with gadolinium contrast enhancement sequence), belonging to the dataset of patient G-13 at the time of diagnosis. 


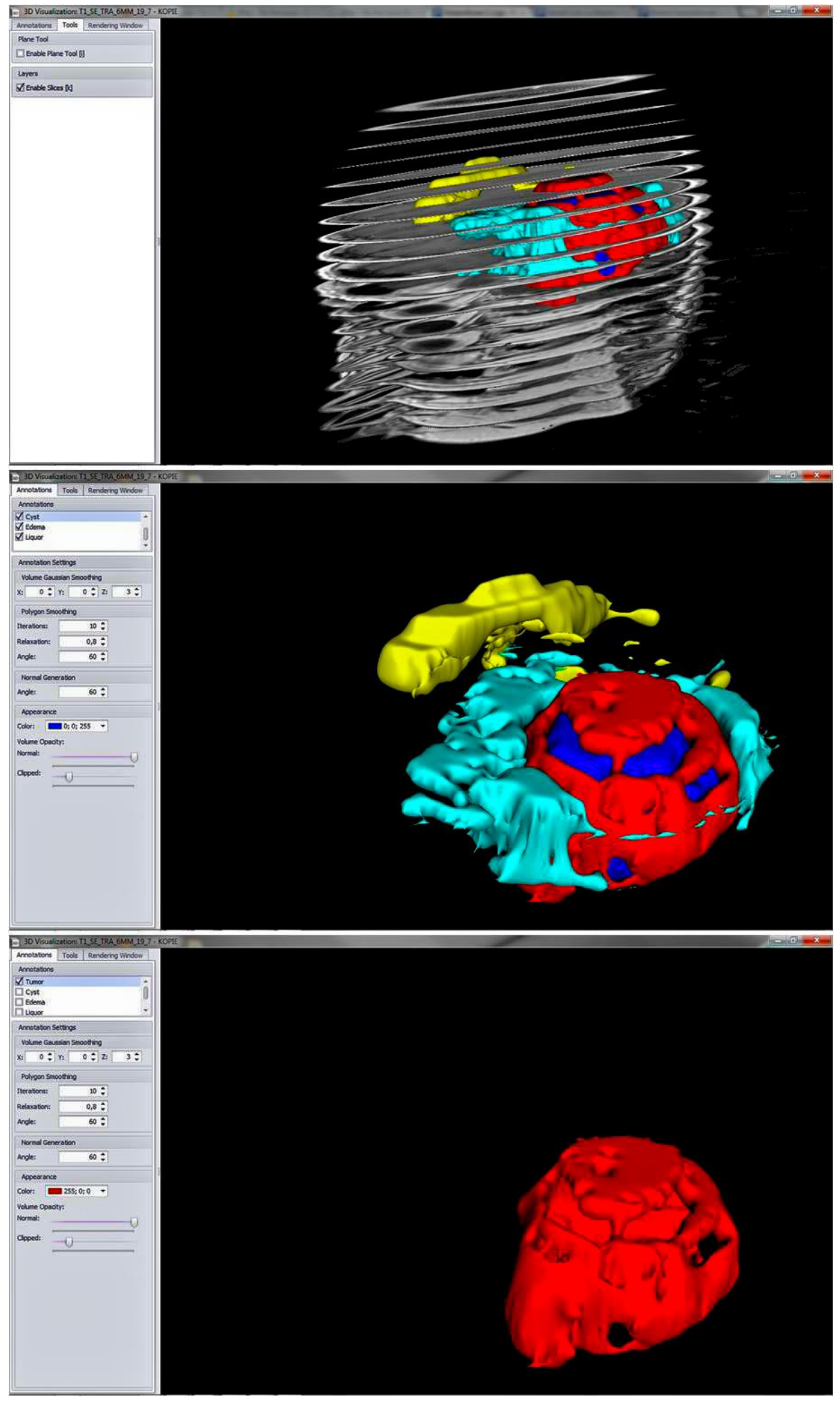

Figure 13: 3D-Vizualization created by DoctorEye

Upper image: Annotated areas surrounded by all of the 19 MRI slides.

Middle image: Annotated areas with active tumor shown in red, necrosis shown in dark blue, edema shown in light blue and cerebrospinal fluid shown in yellow.

Lower image: Isolated active tumor shown in red. 


\subsection{Evaluation of the Histograms}

It is well known that different MR sequences show different shapes of a GBM in a single patient at the same time, as depicted in Figure 14, which makes correct segmentations of tumors nearly impossible. It can be shown that by measuring volumes and segmenting the tumor in different sequences, active tumor-tissue, necrotic areas and edema could be distinguished the best by using the histograms of the signal intensities. These are different within the tumor and vary distinctly in all sequences. Using combinations of histograms from different sequences the tumor can be described in a much better way than by calculating solely the tumor volume.

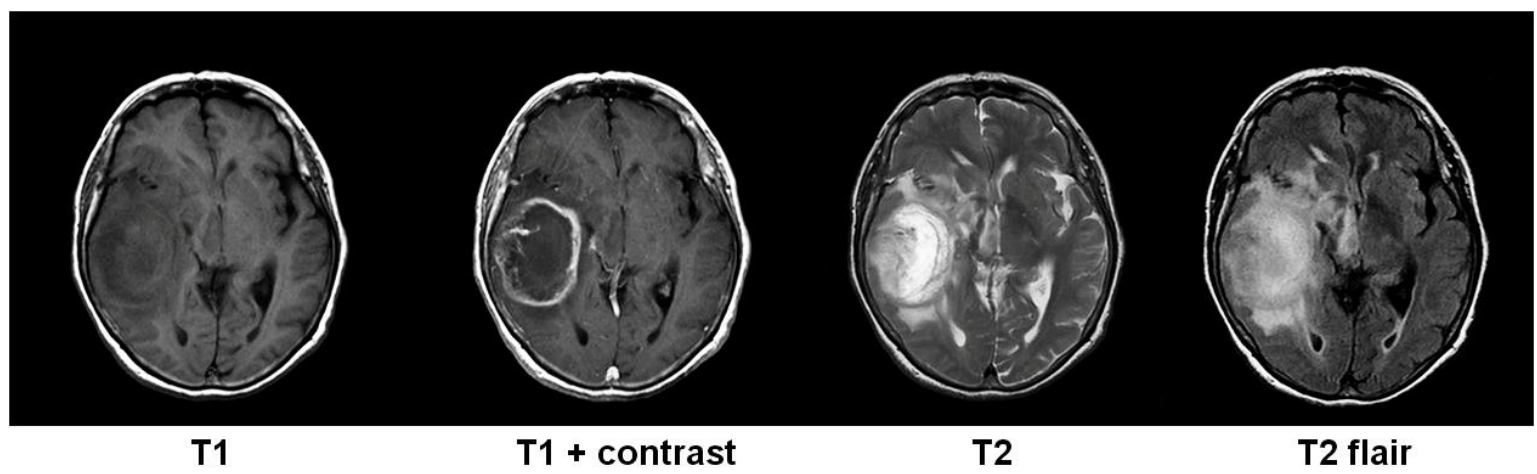

Figure 14: Different Sequences of MR Images in a single Patient with Glioblastoma at the Time of Diagnosis

This image is taken from patient G-13 at the time of diagnosis and depicts how the pictorial representation of intracranial lesions may differ depending on the MR sequence examined.

\subsubsection{At Diagnosis}

In Figure 15 the rendered images of an exemplary patient (G-13), created at the time of diagnosis, are given along with the corresponding histograms. It already emphasizes that the areas of the different tumorous tissues vary strongly in their SI distribution. The same applies for Table III, which demonstrates the mathematical analysis of the segmentation-based histograms. In Figure 16 and Table IV the mean histograms calculated from all patients of the study group (except the 4 non-glioblastomas) and their mathematical analysis are depicted. Also in this summary it is obvious that the SI-distribution of the different qualities 
from the tumorous lesion act different, depending on the MR-sequence investigated. Also the SI-peak, the mean SI and the standardized SI of the ActiveTumor-histogram vary strongly from the necrotic and edematous parts (Table IV) at the time of diagnosis. 

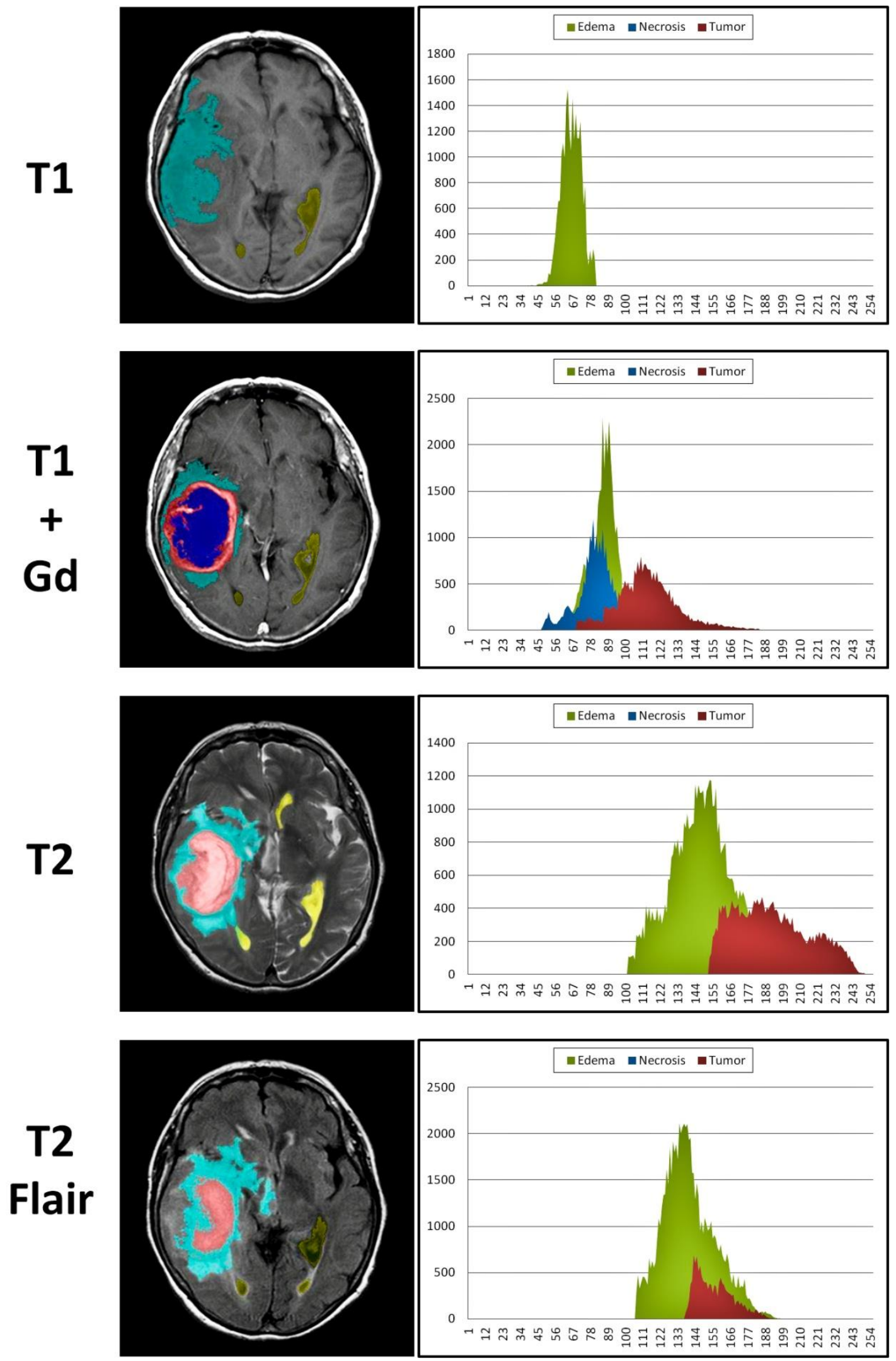

Figure 15: Exemplary annotated DICOM Files of all Sequences from Patient G-13 at the Time of Diagnosis with corresponding Histograms of the whole Set. 


\section{Table III: Exemplary Excel spreadsheet demonstrating the mathematical Analysis of Signalintensity Histograms}

This spreadsheet exemplary shows the mathematical analysis of the histograms acquired from the annotations rendered in the MRI set of Patient G-13 at the time of diagnosis, presenting the results of the T1 with gadolinium contrast enhancement sequence.

\begin{tabular}{|l|l|r|r|r|r|r|}
\hline & Tumor & Necrosis & Edema & Whole Lesion & CSF \\
\hline \multirow{2}{*}{ T1 } & Volume of 1 Pixel $\left(\mathrm{mm}^{3}\right)$ & 4,84 & 4,84 & 4,84 & 4,84 & 4,84 \\
\cline { 2 - 7 } & 0 & 0 & 57620 & 57620 & 11549 \\
\hline Total Pixel-Count & $\mathbf{0}$ & $\mathbf{0}$ & $\mathbf{2 7 9 . 0 6 2}$ & $\mathbf{2 7 9 . 0 6 2}$ & $\mathbf{5 5 . 9 3 3}$ \\
Total Volume $\left(\mathrm{mm}^{3}\right)$ & 0 & 0 & 5009 & 5009 & 413 \\
Maximum Pixel-Count & - & - & 85 & 85 & 46 \\
Signalintensity-Peak & - & - & 85,2 & 85,2 & 62,7 \\
Mean Signalintesity & - & - & $\mathbf{1 , 3 6}$ & $\mathbf{1 , 3 6}$ & $\mathbf{1 , 0 0}$ \\
\hline
\end{tabular}

\begin{tabular}{|c|c|c|c|c|c|c|}
\hline & & Tumor & Necrosis & Edema & Whole Lesion & CSF \\
\hline & Volume of 1 Pixel $\left(\mathrm{mm}^{3}\right)$ & 4,84 & 4,84 & 4,84 & 4,84 & 4,84 \\
\hline & Total Pixel-Count & 16310 & 15956 & 22553 & 54819 & 7517 \\
\hline & Total Volume $\left(\mathrm{mm}^{3}\right)$ & 78.992 & 77.277 & 109.227 & 265.496 & 36.406 \\
\hline & Maximum Pixel-Count & 420 & 1299 & 1531 & 2830 & 500 \\
\hline & Signalintensity-Peak & 93 & 63 & 63 & 63 & 42 \\
\hline & Mean Signalintesity & 102,6 & 63,8 & 65,4 & 76,0 & 49,3 \\
\hline & Standardization to CSF & 2,08 & 1,29 & 1,33 & 1,54 & 1,00 \\
\hline
\end{tabular}

\begin{tabular}{|c|c|c|c|c|c|c|}
\hline & & Tumor & Necrosis & Edema & whole lesion & CSF \\
\hline & Volume of 1 Pixel $\left(\mathrm{mm}^{3}\right)$ & 4,84 & 4,84 & 4,84 & 4,84 & 4,84 \\
\hline & Total Pixel-Count & 27286 & 0 & 52724 & 80010 & 15301 \\
\hline & Total Volume $\left(\mathrm{mm}^{3}\right)$ & 131.986 & 0 & 255.032 & 387.018 & 74.013 \\
\hline 1 & Maximum Pixel-Count & 470 & 0 & 1176 & 1416 & 265 \\
\hline & Signalintensity-Peak & 185 & - & 152 & 156 & 188 \\
\hline & Mean Signalintesity & 191,2 & - & 148,2 & 162,9 & 164,2 \\
\hline & Standardization to CSF & 1,16 & - & 0,90 & 0,99 & 1,00 \\
\hline
\end{tabular}

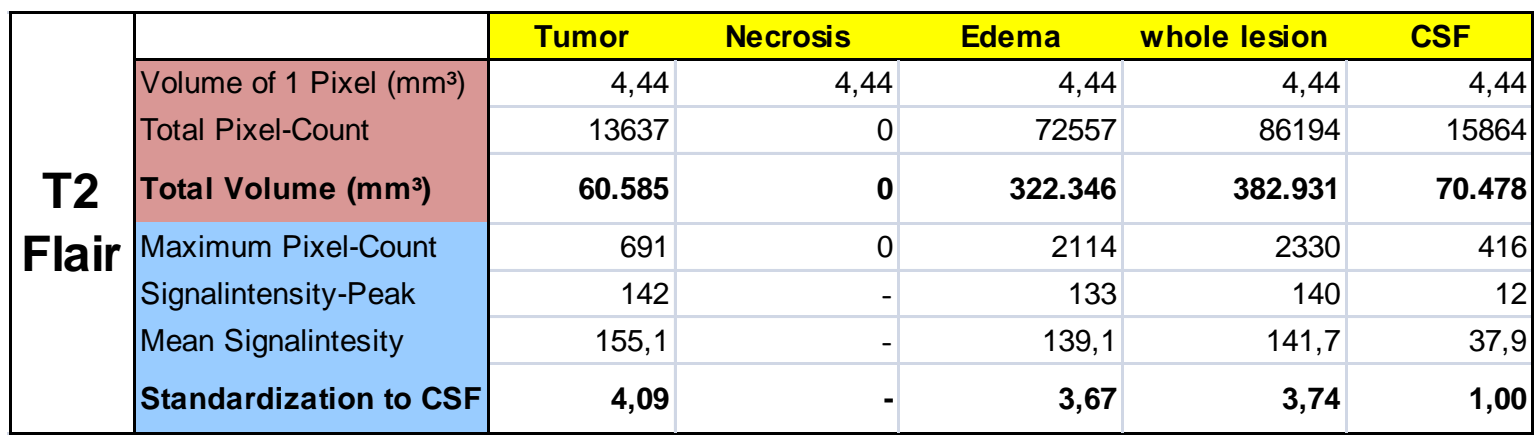



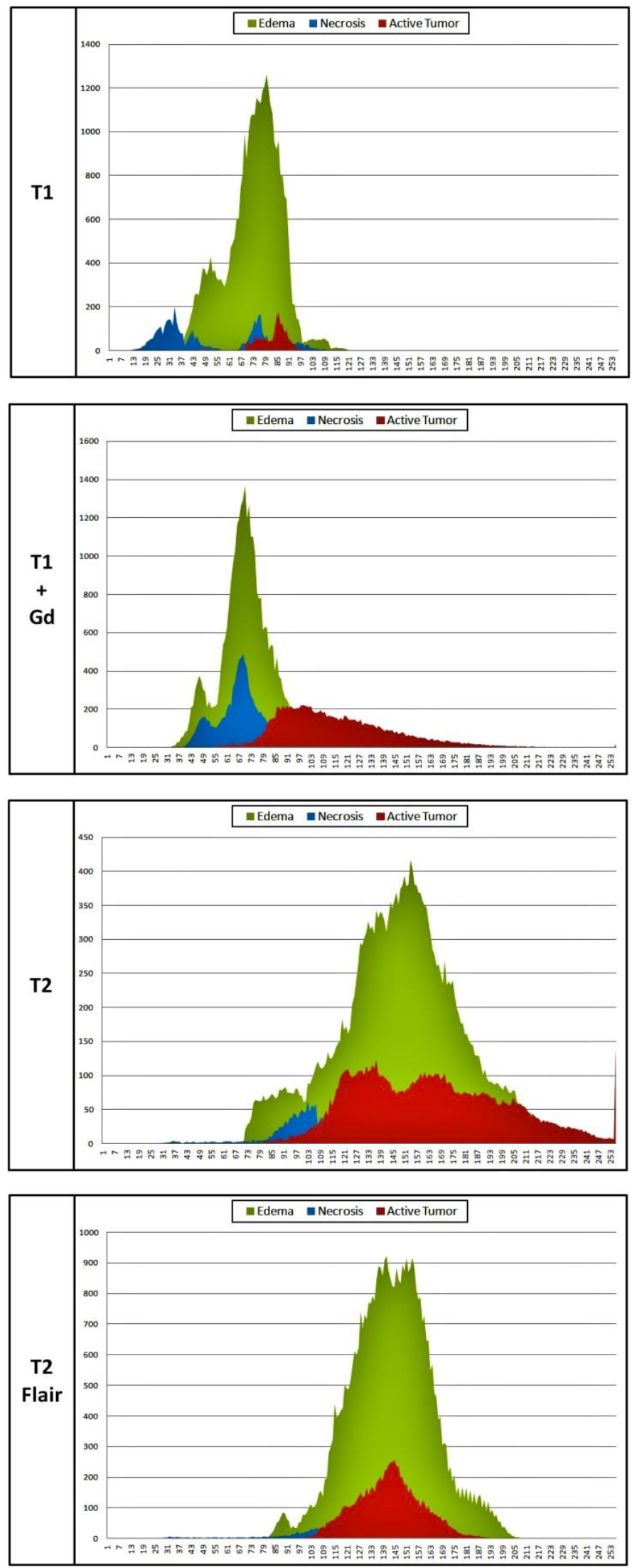

Figure 16: Mean Histogram calculated from all Patients at the Time of Diagnosis 
Table IV: Results from mathematical Analysis of the mean Histograms at the Time of Diagnosis

\begin{tabular}{|l|l|r|r|r|r|r|}
\hline & Tumor & Necrosis & Edema & Whole Lesion & CSF \\
\hline \multirow{2}{*}{ T1 } & Volume of 1 Pixel $\left(\mathrm{mm}^{3}\right)$ & 4,29 & 4,29 & 4,29 & 4,29 & 4,29 \\
\cline { 2 - 7 } & 1696 & 4221 & 35651 & 41567 & 12701 \\
Total Pixel-Count & $\mathbf{7 . 2 7 3}$ & $\mathbf{1 8 . 1 0 7}$ & $\mathbf{1 5 2 . 9 2 6}$ & $\mathbf{1 7 8 . 3 0 6}$ & $\mathbf{5 4 . 4 8 2}$ \\
Total Volume $\left(\mathrm{mm}^{3}\right)$ & 174 & 198 & 1263 & 1385 & 600 \\
Maximum Pixel-Count & 85 & 33 & 79 & 79 & 53 \\
Signalintensity-Peak & 82,0 & 51,7 & 72,5 & 70,8 & 52,1 \\
Mean Signalintesity & $\mathbf{1 , 5 7}$ & $\mathbf{0 , 9 9}$ & $\mathbf{1 , 3 9}$ & $\mathbf{1 , 3 6}$ & $\mathbf{1 , 0 0}$ \\
\hline
\end{tabular}

\begin{tabular}{|c|l|r|r|r|r|r|}
\hline & Tumor & Necrosis & Edema & Whole Lesion & CSF \\
\hline \multirow{2}{*}{$\mathbf{T 1}$} & Volume of 1 Pixel $\left(\mathrm{mm}^{3}\right)$ & 2,54 & 2,54 & 2,54 & 2,54 & 2,54 \\
\cline { 2 - 7 } & Total Pixel-Count & 13260 & 9742 & 31697 & 54700 & 12042 \\
$\mathbf{G d}$ & Total Volume $\left(\mathrm{mm}^{3}\right)$ & $\mathbf{3 3 . 6 1 7}$ & $\mathbf{2 4 . 6 9 9}$ & $\mathbf{8 0 . 3 5 8}$ & $\mathbf{1 3 8 . 6 7 4}$ & $\mathbf{3 0 . 5 2 9}$ \\
& Maximum Pixel-Count & 221 & 486 & 1372 & 1837 & 630 \\
& Signalintensity-Peak & 88 & 68 & 69 & 69 & 42 \\
& Mean Signalintesity & 116,2 & 67,6 & 68,6 & 80,0 & 51,4 \\
& Standardization to CSF & $\mathbf{2 , 2 6}$ & $\mathbf{1 , 3 2}$ & $\mathbf{1 , 3 4}$ & $\mathbf{1 , 5 6}$ & $\mathbf{1 , 0 0}$ \\
\hline
\end{tabular}

\begin{tabular}{|l|l|r|r|r|r|r|}
\hline & Tumor & Necrosis & Edema & whole lesion & CSF \\
\cline { 2 - 7 } & Volume of 1 Pixel $\left(\mathrm{mm}^{3}\right)$ & 4,12 & 4,12 & 4,12 & 4,12 & 4,12 \\
\cline { 2 - 7 } & Total Pixel-Count & 9863 & 1393 & 25917 & 37173 & 15363 \\
\hline & Total Volume $\left(\mathrm{mm}^{3}\right)$ & $\mathbf{4 0 . 6 2 3}$ & $\mathbf{5 . 7 3 7}$ & $\mathbf{1 0 6 . 7 4 4}$ & $\mathbf{1 5 3 . 1 0 4}$ & $\mathbf{6 3 . 2 7 7}$ \\
& Maximum Pixel-Count & 150 & 62 & 418 & 504 & 815 \\
\cline { 2 - 7 } & Signalintensity-Peak & 255 & 102 & 153 & 153 & 255 \\
Mean Signalintesity & 163,1 & 102,0 & 148,8 & 150,9 & 174,5 \\
Standardization to CSF & $\mathbf{0 , 9 3}$ & $\mathbf{0 , 5 8}$ & $\mathbf{0 , 8 5}$ & $\mathbf{0 , 8 6}$ & $\mathbf{1 , 0 0}$ \\
\hline
\end{tabular}

\begin{tabular}{|c|l|r|r|r|r|r|}
\hline & Tumor & Necrosis & Edema & whole lesion & CSF \\
\cline { 2 - 7 } & Volume of 1 Pixel $\left(\mathrm{mm}^{3}\right)$ & 4,43 & 4,43 & 4,43 & 4,43 & 4,43 \\
\cline { 2 - 7 } & Total Pixel-Count & 8783 & 1561 & 45886 & 56230 & 16388 \\
\hline \multirow{2}{*}{ Flair } & Total Volume $\left(\mathrm{mm}^{3}\right)$ & $\mathbf{3 8 . 8 7 8}$ & $\mathbf{6 . 9 0 9}$ & $\mathbf{2 0 3 . 1 0 4}$ & $\mathbf{2 4 8 . 8 9 1}$ & $\mathbf{7 2 . 5 3 7}$ \\
& Maximum Pixel-Count & 256 & 45 & 923 & 1148 & 438 \\
\cline { 2 - 7 } & Signalintensity-Peak & 144 & 126 & 140 & 140 & 17 \\
& Mean Signalintesity & 141,2 & 104,6 & 142,6 & 141,3 & 34,4 \\
& Standardization to CSF & $\mathbf{4 , 1 0}$ & $\mathbf{3 , 0 4}$ & $\mathbf{4 , 1 4}$ & $\mathbf{4 , 1 0}$ & $\mathbf{1 , 0 0}$ \\
\hline
\end{tabular}




\subsubsection{Follow-up}

In the first part of this section (4.2.2.1 Single Patient) the follow-up of an exemplary patient in all four investigated MR sequences is illustrated. Besides different exemplary MR-Images, the corresponding histograms of the whole set from each time point (at diagnosis, after surgery and after radiation- and chemotherapy) are depicted in Figure 17, showing the results from T1-, Figure 18 from T1 Gd-MRI-, Figure 19 from T2- and Figure 20 from T2 Flair-sequence. According to each sequence and histogram the mathematical analysis is presented in Tables V-VIII.

The second part (4.2.2.2 Summary) follows the same structure as the first, only, instead of presenting a single patient's results, it depicts the mean histograms calculated from all patients of the study group (except the 4 non-glioblastomas) and the corresponding mathematical analysis, shown in Figures 21-24 and Tables IX-XII.

In comparison to the first and second part, the third one (4.2.2.3 NonGlioblastoma) pictures the follow-up of an intracranial tumorous lesion that was proved not to be a glioblastoma (patient G-8; brain metastasis deriving from bronchial cancer). To provide a clear structure only T1 Gd-MRI is given as an exemplary MR-Sequence as you can see in Figure 25. Table XIII displays the corresponding mathematical analysis. 


\subsubsection{Single Patient}
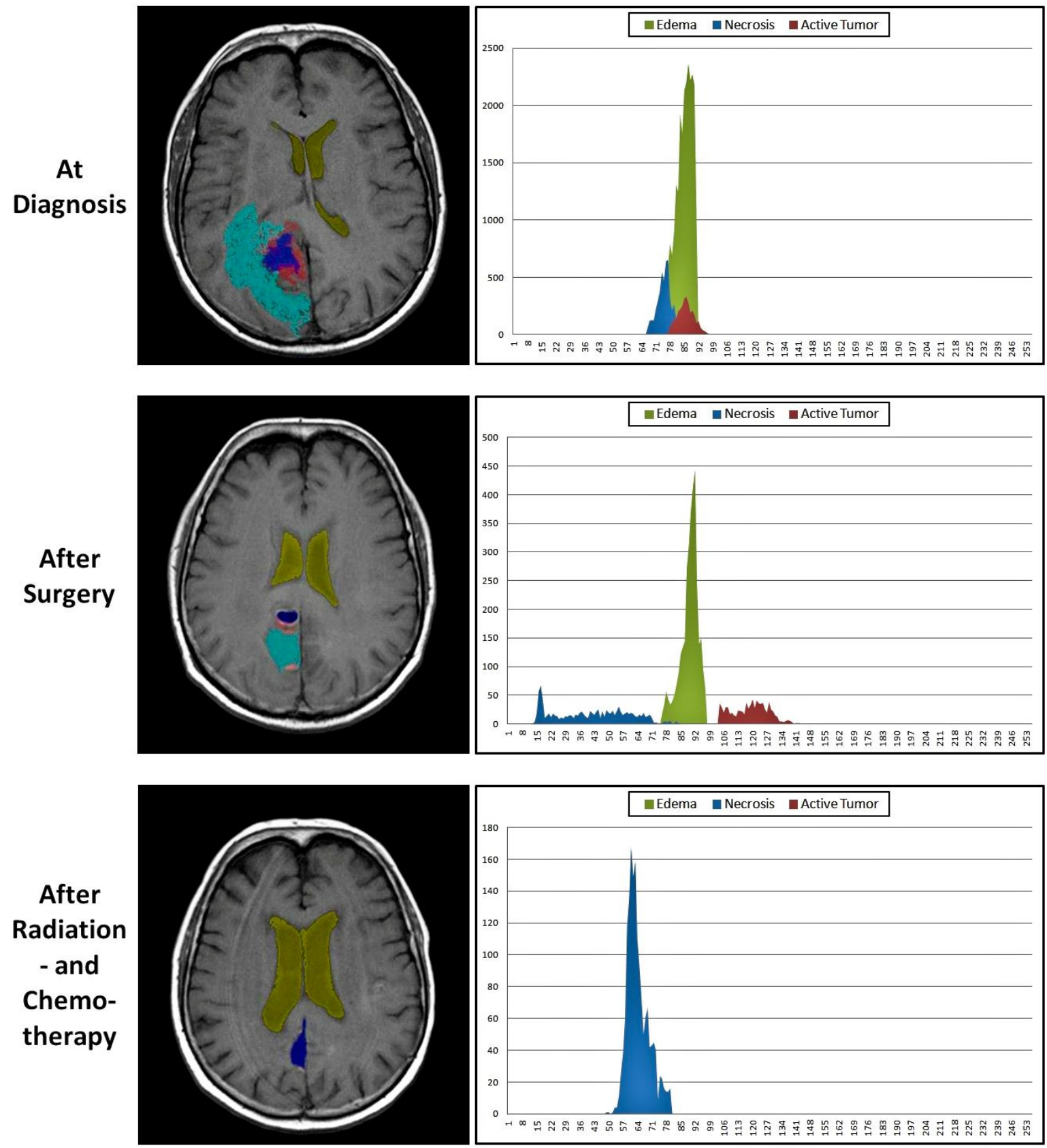

Figure 17: Follow-up of Patient G-25 in T1-Sequence 
Table V: Results from mathematical Analysis of Patient G-25's Histograms from T1-Sequence during Follow-up

\begin{tabular}{|c|c|c|c|c|c|c|}
\hline \multirow{8}{*}{$\begin{array}{c}\text { At } \\
\text { Diagnosis }\end{array}$} & & Tumor & Necrosis & Edema & Whole Lesion & CSF \\
\hline & Volume of $1 \mathrm{Pixel}\left(\mathrm{mm}^{3}\right)$ & 3,05 & 3,05 & 3,05 & 3,05 & 3,05 \\
\hline & Total Pixel-Count & 3038 & 4718 & 25530 & 33286 & 15272 \\
\hline & Total Volume $\left(\mathrm{mm}^{3}\right)$ & 9.271 & 14.398 & 77.911 & 101.581 & 46.606 \\
\hline & Maximum Pixel-Count & 334 & 653 & 2371 & 2652 & 892 \\
\hline & Signalintensity-Peak & 85 & 76 & 86 & 86 & 47 \\
\hline & Mean Signalintesity & 84,8 & 74,2 & 83,5 & 82,3 & 54,2 \\
\hline & Standardization to CSF & 1,56 & 1,37 & 1,54 & 1,52 & 1,00 \\
\hline
\end{tabular}

\begin{tabular}{|c|c|c|c|c|c|c|}
\hline & & Tumor & Necrosis & Edema & Whole Lesion & CSF \\
\hline & Volume of 1 Pixel $\left(\mathrm{mm}^{3}\right)$ & 4,84 & 4,84 & 4,84 & 4,84 & 4,84 \\
\hline & Total Pixel-Count & 818 & 1130 & 3349 & 5297 & 9566 \\
\hline After & Total Volume $\left(\mathrm{mm}^{3}\right)$ & 3.962 & 5.473 & 16.220 & 25.654 & 46.329 \\
\hline \multirow{4}{*}{ Surgery } & Maximum Pixel-Count & 43 & 68 & 444 & 444 & 449 \\
\hline & Signalintensity-Peak & 119 & 16 & 91 & 91 & 50 \\
\hline & Mean Signalintesity & 118,2 & 41,2 & 88,4 & 82,9 & 62,3 \\
\hline & Standardization to CSF & 1,90 & 0,66 & 1,42 & 1,33 & 1,00 \\
\hline
\end{tabular}

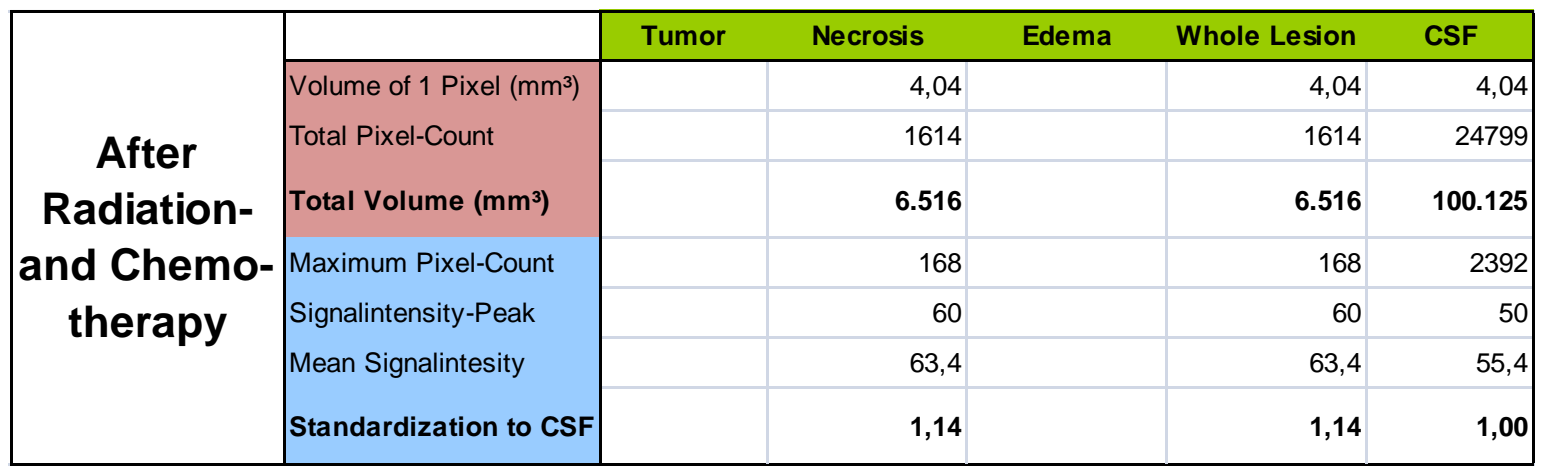



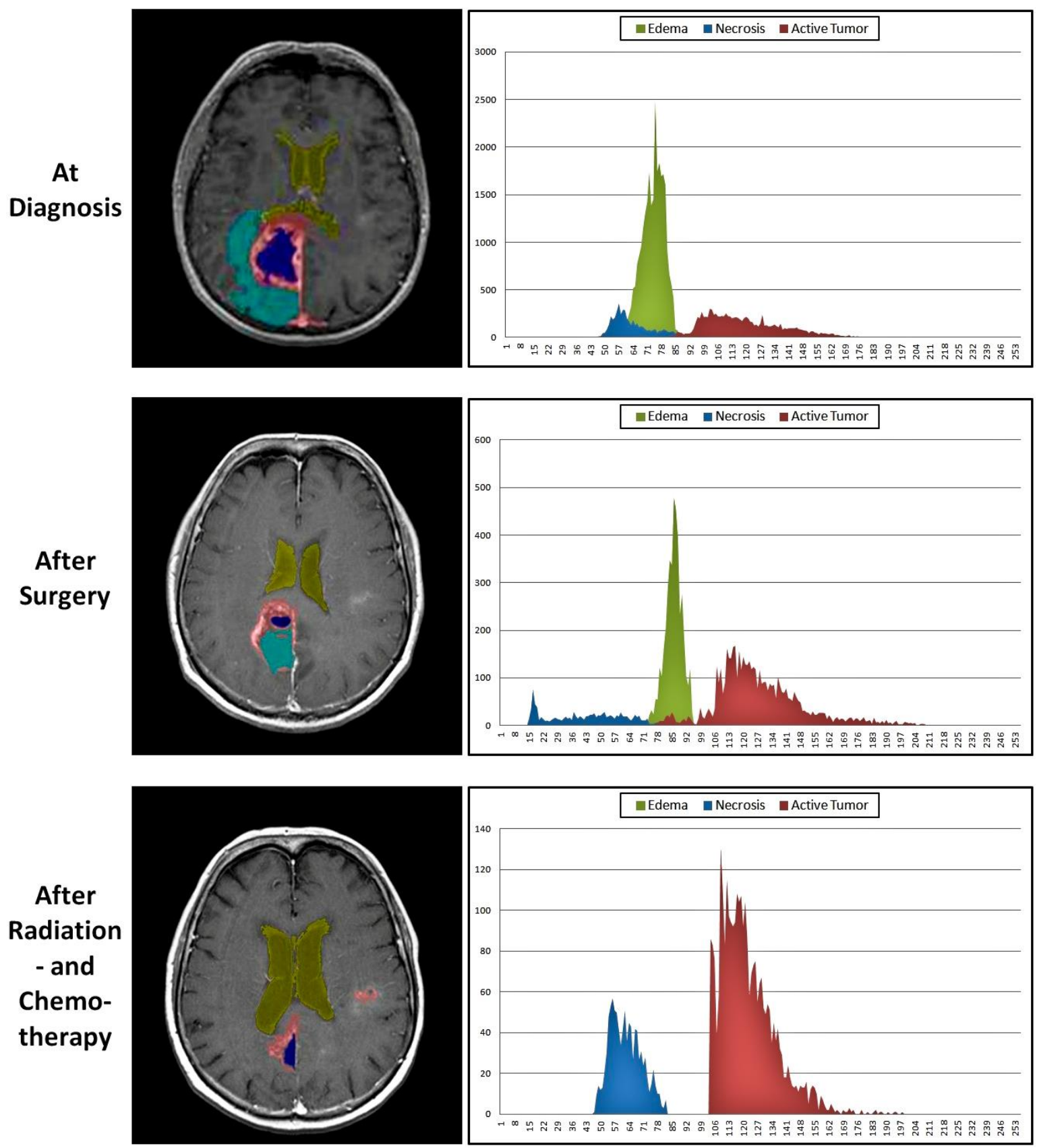

Figure 18: Follow-up of Patient G-25 in T1 with Gadolinium Enhancement Sequence 
Table VI: Results from mathematical Analysis of Patient G-25's Histograms from T1 with Gadolinium Enhancement Sequence during Follow-up

\begin{tabular}{|c|c|c|c|c|c|c|}
\hline \multirow{8}{*}{$\begin{array}{c}\text { At } \\
\text { Diagnosis }\end{array}$} & & Tumor & Necrosis & Edema & Whole Lesion & CSF \\
\hline & Volume of 1 Pixel $\left(\mathrm{mm}^{3}\right)$ & 2,22 & 2,22 & 2,22 & 2,22 & 2,22 \\
\hline & Total Pixel-Count & 10633 & 5116 & 26901 & 42650 & 8433 \\
\hline & Total Volume $\left(\mathrm{mm}^{3}\right)$ & 23.619 & 11.364 & 59.756 & 94.740 & 18.732 \\
\hline & Maximum Pixel-Count & 298 & 357 & 2507 & 2595 & 605 \\
\hline & Signalintensity-Peak & 101 & 56 & 74 & 74 & 39 \\
\hline & Mean Signalintesity & 118,3 & 63,5 & 73,0 & 83,1 & 48,5 \\
\hline & Standardization to CSF & 2,44 & 1,31 & 1,50 & 1,71 & 1,00 \\
\hline
\end{tabular}

\begin{tabular}{|c|c|c|c|c|c|c|}
\hline & & Tumor & Necrosis & Edema & Whole Lesion & CSF \\
\hline & Volume of 1 Pixel $\left(\mathrm{mm}^{3}\right)$ & 4,84 & 4,84 & 4,84 & 4,84 & 4,84 \\
\hline & Total Pixel-Count & 5472 & 1214 & 4144 & 10830 & 10021 \\
\hline After & Total Volume $\left(\mathrm{mm}^{3}\right)$ & 26.502 & 5.880 & 20.070 & 52.451 & 48.533 \\
\hline \multirow{4}{*}{ Surgery } & Maximum Pixel-Count & 167 & 76 & 480 & 500 & 395 \\
\hline & Signalintensity-Peak & 115 & 16 & 85 & 85 & 47 \\
\hline & Mean Signalintesity & 127,3 & 42,9 & 84,8 & 101,6 & 63,8 \\
\hline & Standardization to CSF & 1,99 & 0,67 & 1,33 & 1,59 & 1,00 \\
\hline
\end{tabular}

\begin{tabular}{|c|c|c|c|c|c|c|}
\hline & & Tumor & Necrosis & Edema & Whole Lesion & CSF \\
\hline & Volume of 1 Pixel $\left(\mathrm{mm}^{3}\right)$ & 4,04 & 4,04 & & 4,04 & 4,04 \\
\hline After & Total Pixel-Count & 2943 & 986 & & 3929 & 22102 \\
\hline Radiation- & Total Volume $\left(\mathrm{mm}^{3}\right)$ & 11.882 & 3.981 & & 15.863 & 89.236 \\
\hline and Chemo- & Maximum Pixel-Count & 131 & 57 & & 131 & 2105 \\
\hline theranv & Signalintensity-Peak & 108 & 55 & & 108 & 47 \\
\hline & Mean Signalintesity & 121,2 & 61,5 & & 106,2 & 52,7 \\
\hline & Standardization to CSF & 2,30 & 1,17 & & 2,02 & 1,00 \\
\hline
\end{tabular}



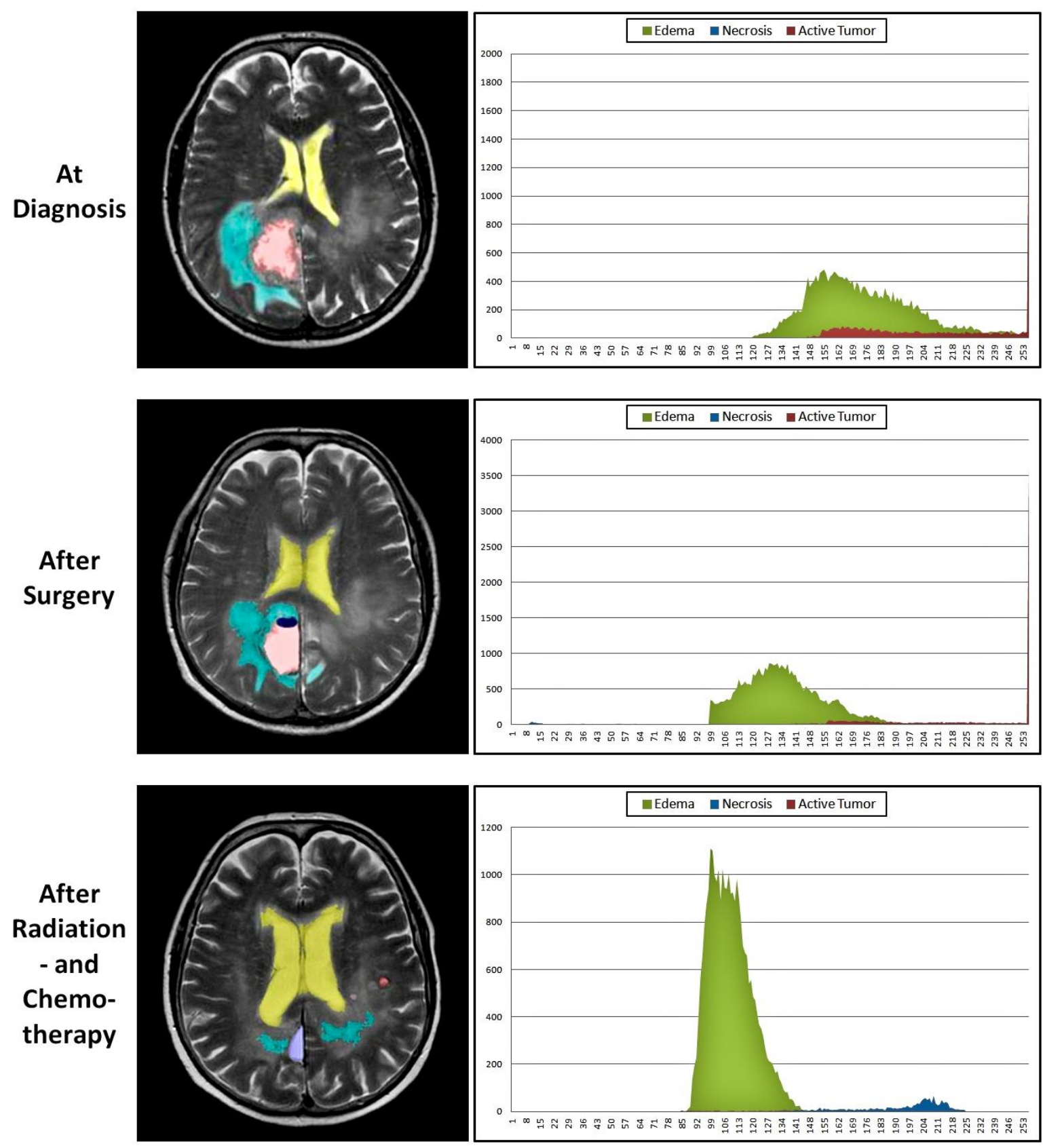

Figure 19: Follow-up of Patient G-25 in T2 Sequence 
Table VII: Results from mathematical Analysis of Patient G-25's Histograms from T2-Sequence during Follow-up

\begin{tabular}{|c|c|c|c|c|c|c|}
\hline \multirow{8}{*}{$\begin{array}{c}\text { At } \\
\text { Diagnosis }\end{array}$} & & Tumor & Necrosis & Edema & Whole Lesion & CSF \\
\hline & Volume of 1 Pixel $\left(\mathrm{mm}^{3}\right)$ & 3,37 & & 3,37 & 3,37 & 3,37 \\
\hline & Total Pixel-Count & 6633 & & 26458 & 33091 & 8121 \\
\hline & Total Volume $\left(\mathrm{mm}^{3}\right)$ & 22.348 & & 89.141 & 111.488 & 27.361 \\
\hline & Maximum Pixel-Count & 1809 & & 483 & 1938 & 3465 \\
\hline & Signalintensity-Peak & 255 & & 154 & 255 & 255 \\
\hline & Mean Signalintesity & 211,8 & & 174,1 & 181,6 & 230,9 \\
\hline & Standardization to CSF & 0,92 & & 0,75 & 0,79 & 1,00 \\
\hline
\end{tabular}

\begin{tabular}{|c|c|c|c|c|c|c|}
\hline & & Tumor & Necrosis & Edema & Whole Lesion & CSF \\
\hline & Volume of 1 Pixel $\left(\mathrm{mm}^{3}\right)$ & 4,84 & 4,84 & 4,84 & 4,84 & 4,84 \\
\hline & Total Pixel-Count & 7436 & 818 & 39150 & 47404 & 11482 \\
\hline After & Total Volume $\left(\mathrm{mm}^{3}\right)$ & 35.969 & 3.957 & 189.373 & 229.299 & 55.540 \\
\hline \multirow{4}{*}{ Surgery } & Maximum Pixel-Count & 3756 & 42 & 870 & 3756 & 185 \\
\hline & Signalintensity-Peak & 255 & 10 & 127 & 255 & 200 \\
\hline & Mean Signalintesity & 224,8 & 39,9 & 133,7 & 146,4 & 181,9 \\
\hline & Standardization to CSF & 1,24 & 0,22 & 0,73 & 0,80 & 1,00 \\
\hline
\end{tabular}

\begin{tabular}{|c|l|r|r|r|r|r|}
\hline & & Tumor & Necrosis & Edema & Whole Lesion & CSF \\
\cline { 2 - 7 } $\begin{array}{c}\text { After } \\
\text { Radiation- }\end{array}$ & Volume of 1 Pixel $\left(\mathrm{mm}^{3}\right)$ & 4,04 & 4,04 & 4,04 & 4,04 & 4,04 \\
and Chemo- & Total Pixel-Count & 118 & 1388 & 27993 & 29499 & 33150 \\
theral Volume $\left(\mathbf{m m}^{3}\right)$ & $\mathbf{4 7 7}$ & $\mathbf{5 . 6 1 2}$ & $\mathbf{1 1 3 . 1 7 5}$ & $\mathbf{1 1 9 . 2 6 4}$ & $\mathbf{1 3 4 . 0 2 4}$ \\
& Maximum Pixel-Count & 7 & 65 & 1112 & 1114 & 1420 \\
\hline & Signalintensity-Peak & 134 & 208 & 98 & 98 & 172 \\
\hline & Mean Signalintesity & 113,2 & 192,6 & 107,9 & 111,9 & 162,8 \\
\hline & Standardization to CSF & $\mathbf{0 , 7 0}$ & $\mathbf{1 , 1 8}$ & $\mathbf{0 , 6 6}$ & $\mathbf{0 , 6 9}$ & $\mathbf{1 , 0 0}$ \\
\hline
\end{tabular}



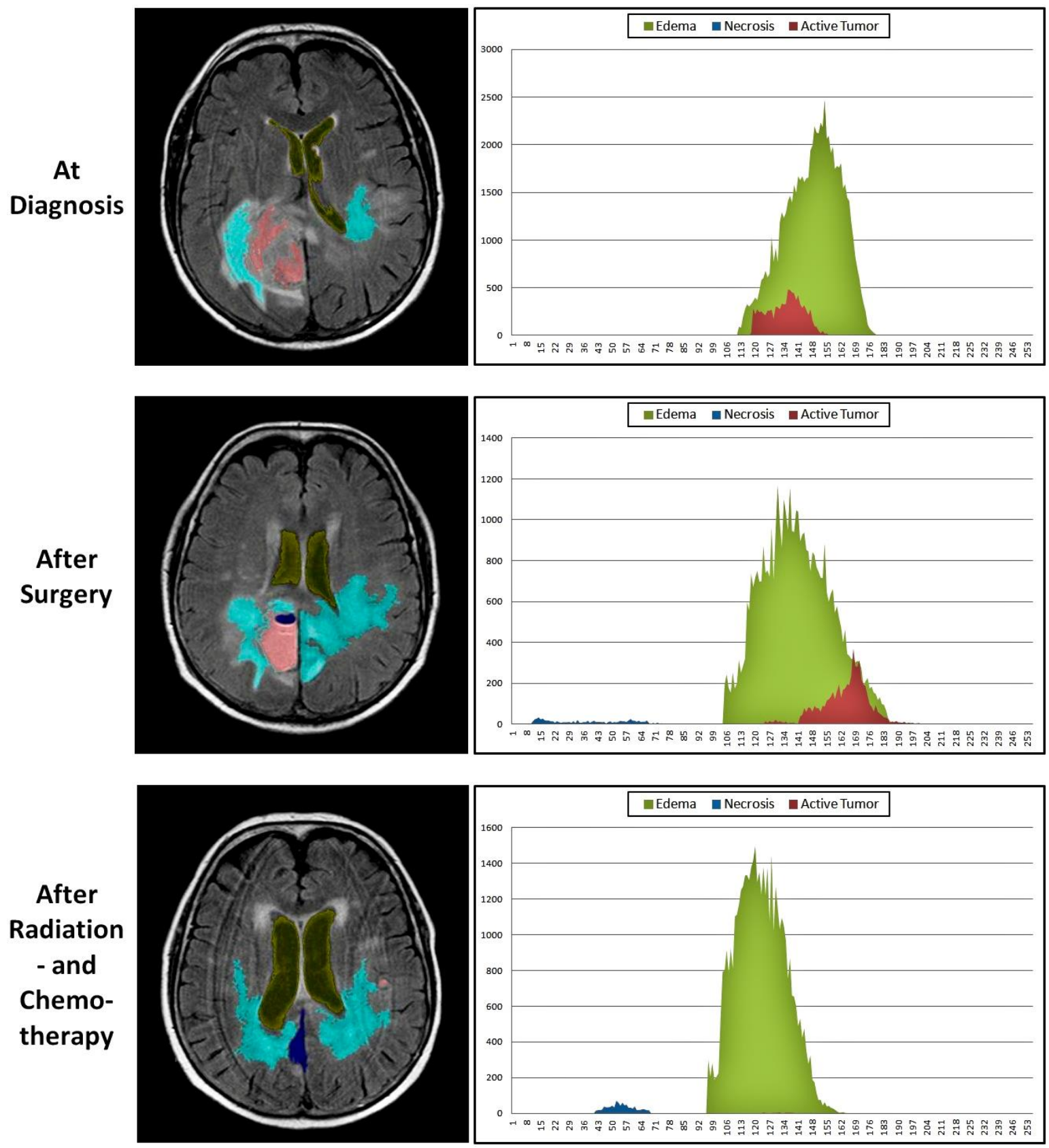

Figure 20: Follow-up of Patient G-25 in T2 Flair Sequence 
Table VIII: Results from mathematical Analysis of Patient G-25's Histograms from T2 Flair-Sequence during Follow-up

\begin{tabular}{|c|c|c|c|c|c|c|}
\hline \multirow{8}{*}{$\begin{array}{c}\text { At } \\
\text { Diagnosis }\end{array}$} & & Tumor & Necrosis & Edema & Whole Lesion & CSF \\
\hline & Volume of 1 Pixel $\left(\mathrm{mm}^{3}\right)$ & 3,37 & & 3,37 & 3,37 & 3,37 \\
\hline & Total Pixel-Count & 9443 & & 76159 & 85602 & 21727 \\
\hline & Total Volume $\left(\mathrm{mm}^{3}\right)$ & 31.815 & & 256.590 & 288.405 & 73.201 \\
\hline & Maximum Pixel-Count & 482 & & 2478 & 2496 & 628 \\
\hline & Signalintensity-Peak & 135 & & 153 & 153 & 18 \\
\hline & Mean Signalintesity & 133,8 & & 147,0 & 145,6 & 36,3 \\
\hline & Standardization to CSF & 3,68 & & 4,05 & 4,01 & 1,00 \\
\hline
\end{tabular}

\begin{tabular}{|c|l|r|r|r|r|r|}
\hline & \multicolumn{1}{|c|}{ Tumor } & Necrosis & Edema & Whole Lesion & CSF \\
\cline { 2 - 7 } & Volume of 1 Pixel $\left(\mathrm{mm}^{3}\right)$ & 4,84 & 4,84 & 4,84 & 4,84 & 4,84 \\
\cline { 2 - 7 } After & Total Pixel-Count & 5976 & 868 & 45696 & 52540 & 11664 \\
& Total Volume $\left(\mathbf{m m}^{3}\right)$ & $\mathbf{2 8 . 9 0 7}$ & $\mathbf{4 . 1 9 9}$ & $\mathbf{2 2 1 . 0 3 7}$ & $\mathbf{2 5 4 . 1 4 2}$ & $\mathbf{5 6 . 4 2 0}$ \\
& Maximum Pixel-Count & 373 & 33 & 1172 & 1183 & 315 \\
& Signalintensity-Peak & 167 & 13 & 130 & 130 & 17 \\
& Mean Signalintesity & 163,4 & 40,0 & 139,6 & 140,6 & 36,3 \\
& Standardization to CSF & $\mathbf{4 , 5 1}$ & $\mathbf{1 , 1 0}$ & $\mathbf{3 , 8 5}$ & $\mathbf{3 , 8 8}$ & $\mathbf{1 , 0 0}$ \\
\hline
\end{tabular}

\begin{tabular}{|c|l|r|r|r|r|r|}
\hline & \multicolumn{1}{|c|}{ Tumor } & Necrosis & Edema & Whole Lesion & CSF \\
\cline { 2 - 7 } $\begin{array}{c}\text { After } \\
\text { Radiation- }\end{array}$ & Volume of 1 Pixel $\left(\mathrm{mm}^{3}\right)$ & 4,04 & 4,04 & 4,04 & 4,04 & 4,04 \\
and Chemo- & Total Pixel-Count & 53 & 899 & 46496 & 47448 & 25902 \\
therapy & Motal Volume $\left(\mathbf{m m}^{3}\right)$ & $\mathbf{2 1 4}$ & $\mathbf{3 . 6 3 5}$ & $\mathbf{1 8 7 . 9 8 2}$ & $\mathbf{1 9 1 . 8 3 1}$ & $\mathbf{1 0 4 . 7 2 1}$ \\
& Signalintensity-Peak & 6 & 72 & 1500 & 1500 & 1812 \\
& Mean Signalintesity & 131 & 51 & 119 & 119 & 16 \\
& Standardization to CSF & $\mathbf{4 , 5 8}$ & $\mathbf{1 , 8 3}$ & $\mathbf{4 , 1 7}$ & $\mathbf{4 , 1 3}$ & $\mathbf{1 , 0 0}$ \\
\hline
\end{tabular}




\subsubsection{Summary}
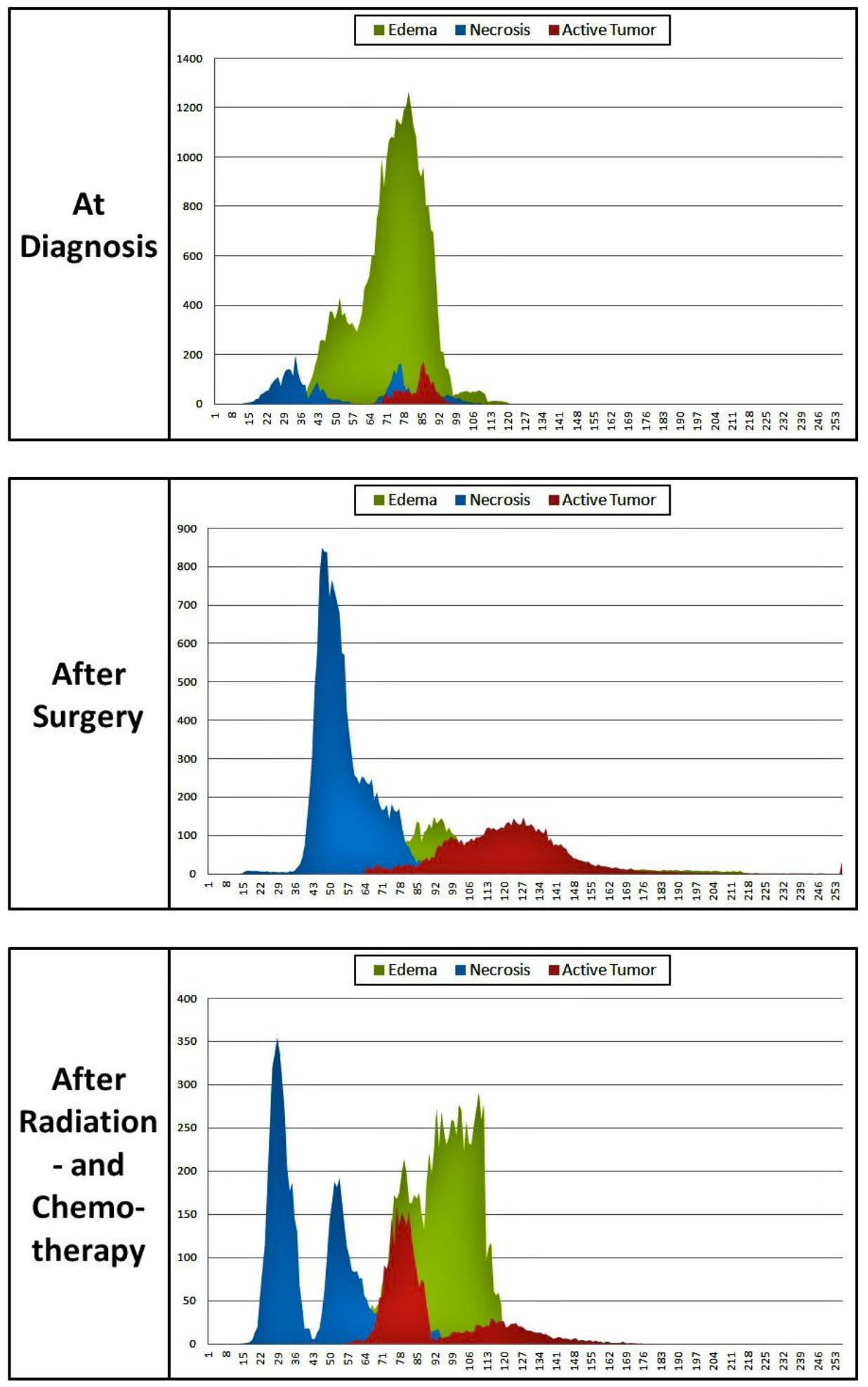

Figure 21: Mean Histogram from T1-Sequence during Follow-up 
Table IX: Results from mathematical Analysis of the mean Histograms from T1-Sequence during Follow-up

\begin{tabular}{|c|l|r|r|r|r|r|}
\hline & \multicolumn{1}{|c|}{ Tumor } & Necrosis & Edema & Whole Lesion & \multicolumn{1}{c|}{ CSF } \\
\cline { 2 - 7 } At & Volume of 1 Pixel $\left(\mathrm{mm}^{3}\right)$ & 4,29 & 4,29 & 4,29 & 4,29 & 4,29 \\
\cline { 2 - 7 } Diagnosis & 1696 & 4221 & 35651 & 41567 & 12701 \\
& Total Pixel-Count & $\mathbf{7 . 2 7 3}$ & $\mathbf{1 8 . 1 0 7}$ & $\mathbf{1 5 2 . 9 2 6}$ & $\mathbf{1 7 8 . 3 0 6}$ & $\mathbf{5 4 . 4 8 2}$ \\
& Total Volume $\left(\mathbf{m m}^{3}\right)$ & 174 & 198 & 1263 & 1385 & 600 \\
& Maximum Pixel-Count & 85 & 33 & 79 & 79 & 53 \\
\cline { 2 - 7 } & Signalintensity-Peak & 82,0 & 51,7 & 72,5 & 70,8 & 52,1 \\
& Mean Signalintesity & $\mathbf{1 , 5 7}$ & $\mathbf{0 , 9 9}$ & $\mathbf{1 , 3 9}$ & $\mathbf{1 , 3 6}$ & $\mathbf{1 , 0 0}$ \\
\hline
\end{tabular}

\begin{tabular}{|c|c|c|c|c|c|c|}
\hline & & Tumor & Necrosis & Edema & Whole Lesion & CSF \\
\hline & Volume of 1 Pixel $\left(\mathrm{mm}^{3}\right)$ & 4,06 & 4,06 & 4,06 & 4,06 & 4,06 \\
\hline & Total Pixel-Count & 7009 & 15735 & 5480 & 28224 & 14078 \\
\hline After & Total Volume $\left(\mathrm{mm}^{3}\right)$ & 28.443 & 63.850 & 22.236 & 114.529 & 57.126 \\
\hline \multirow[t]{4}{*}{ Surgery } & Maximum Pixel-Count & 147 & 850 & 149 & 850 & 724 \\
\hline & Signalintensity-Peak & 127 & 46 & 91 & 46 & 50 \\
\hline & Mean Signalintesity & 121,3 & 54,8 & 102,2 & 80,5 & 61,2 \\
\hline & Standardization to CSF & 1,98 & 0,89 & 1,67 & 1,32 & 1,00 \\
\hline
\end{tabular}

\begin{tabular}{|c|l|r|r|r|r|r|}
\hline & \multicolumn{1}{|c|}{ Tumor } & \multicolumn{1}{c|}{ Necrosis } & Edema & Whole Lesion & CSF \\
\cline { 2 - 7 } After & Volume of 1 Pixel $\left(\mathrm{mm}^{3}\right)$ & 3,95 & 3,95 & 3,95 & 3,95 & 3,95 \\
Radiation- & Total Pixel-Count & 3145 & 6599 & 9519 & 19263 & 12997 \\
$\begin{array}{c}\text { and Chemo- } \\
\text { therapy }\end{array}$ & Total Volume $\left(\mathbf{m m}^{3}\right)$ & $\mathbf{1 2 . 4 3 4}$ & $\mathbf{2 6 . 0 9 0}$ & $\mathbf{3 7 . 6 3 8}$ & $\mathbf{7 6 . 1 6 2}$ & $\mathbf{5 1 . 3 8 9}$ \\
& Maximum Pixel-Count & 159 & 355 & 291 & 381 & 651 \\
& Signalintensity-Peak & 76 & 28 & 109 & 79 & 51 \\
\hline & Mean Signalintesity & 90,0 & 43,2 & 93,7 & 75,8 & 56,6 \\
\hline
\end{tabular}



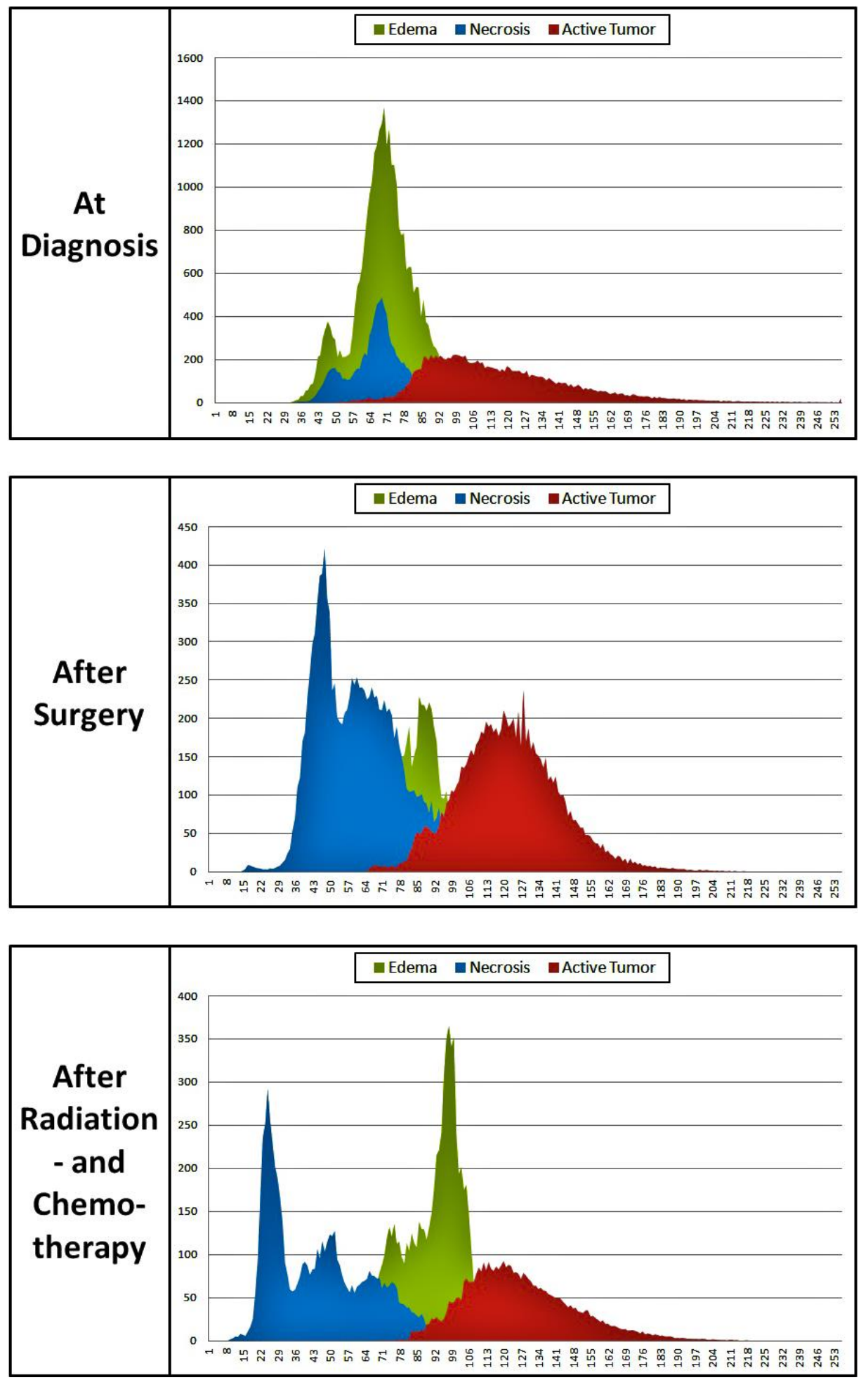

Figure 22: Mean Histogram from T1 with Gadolinium Contrast Enhancement during Followup 
Table X: Results from mathematical Analysis of the mean Histograms from T1 with Gadolinium Contrast Enhancement during Follow-up

\begin{tabular}{|c|l|r|r|r|r|r}
\hline & \multicolumn{1}{|c|}{ Tumor } & Necrosis & Edema & Whole Lesion & \multicolumn{1}{c}{ CSF } \\
\cline { 2 - 7 } At & Volume of 1 Pixel $\left(\mathrm{mm}^{3}\right)$ & 2,54 & 2,54 & 2,54 & 2,54 & 2,54 \\
\hline \multirow{2}{*}{ Diagnosis } & 13260 & 9742 & 31697 & 54700 & 12042 \\
\hline & Total Pixel-Count & $\mathbf{3 3 . 6 1 7}$ & $\mathbf{2 4 . 6 9 9}$ & $\mathbf{8 0 . 3 5 8}$ & $\mathbf{1 3 8 . 6 7 4}$ & $\mathbf{3 0 . 5 2 9}$ \\
\hline & Total Volume $\left(\mathbf{m m}^{3}\right)$ & 221 & 486 & 1372 & 1837 & 630 \\
\hline & Maximum Pixel-Count & 88 & 68 & 69 & 69 & 42 \\
\hline & Signalintensity-Peak & 116,2 & 67,6 & 68,6 & 80,0 & 51,4 \\
\hline & Mean Signalintesity & $\mathbf{2 , 2 6}$ & $\mathbf{1 , 3 2}$ & $\mathbf{1 , 3 4}$ & $\mathbf{1 , 5 6}$ & $\mathbf{1 , 0 0}$ \\
\hline
\end{tabular}

\begin{tabular}{|c|l|r|r|r|r|r|}
\hline & \multicolumn{1}{|c|}{ Tumor } & Necrosis & Edema & Whole Lesion & \multicolumn{1}{c}{ CSF } \\
\cline { 2 - 7 } & Volume of 1 Pixel $\left(\mathrm{mm}^{3}\right)$ & 3,82 & 3,82 & 3,82 & 3,82 & 3,82 \\
\cline { 2 - 7 } After & Total Pixel-Count & 9702 & 13109 & 8455 & 31266 & 13778 \\
\hline & Total Volume $\left(\mathbf{m m}^{3}\right)$ & $\mathbf{3 7 . 0 9 6}$ & $\mathbf{5 0 . 1 2 6}$ & $\mathbf{3 2 . 3 3 1}$ & $\mathbf{1 1 9 . 5 5 3}$ & $\mathbf{5 2 . 6 8 2}$ \\
\hline & Maximum Pixel-Count & 238 & 422 & 229 & 491 & 578 \\
\hline & Signalintensity-Peak & 127 & 47 & 85 & 47 & 46 \\
\hline & Mean Signalintesity & 121,4 & 63,1 & $\mathbf{7 4 , 4}$ & 84,2 & 53,0 \\
\hline & Standardization to CSF & $\mathbf{2 , 2 9}$ & $\mathbf{1 , 1 9}$ & $\mathbf{1 , 4 0}$ & $\mathbf{1 , 5 9}$ & $\mathbf{1 , 0 0}$ \\
\hline
\end{tabular}

\begin{tabular}{|c|l|r|r|r|r|r|}
\hline & \multicolumn{1}{|c|}{ Tumor } & Necrosis & Edema & Whole Lesion & CSF \\
\cline { 2 - 7 } After & Volume of 1 Pixel $\left(\mathrm{mm}^{3}\right)$ & 3,89 & 3,89 & 3,89 & 3,89 & 3,89 \\
\hline $\begin{array}{c}\text { Radiation- } \\
\text { and Chemo- }\end{array}$ & Total Pixel-Count & 4417 & 6653 & 6869 & 17938 & 12416 \\
therapy & Total Volume $\left(\mathbf{m m}^{3}\right)$ & $\mathbf{1 7 . 1 9 8}$ & $\mathbf{2 5 . 9 0 8}$ & $\mathbf{2 6 . 7 4 6}$ & $\mathbf{6 9 . 8 5 2}$ & $\mathbf{4 8 . 3 4 7}$ \\
\hline & Maximum Pixel-Count & 93 & 292 & 366 & 427 & 570 \\
\hline & Signalintensity-Peak & 119 & 24 & 97 & 97 & 48 \\
\hline & Mean Signalintesity & 125,5 & 46,3 & 89,7 & 82,4 & 54,5 \\
\hline
\end{tabular}



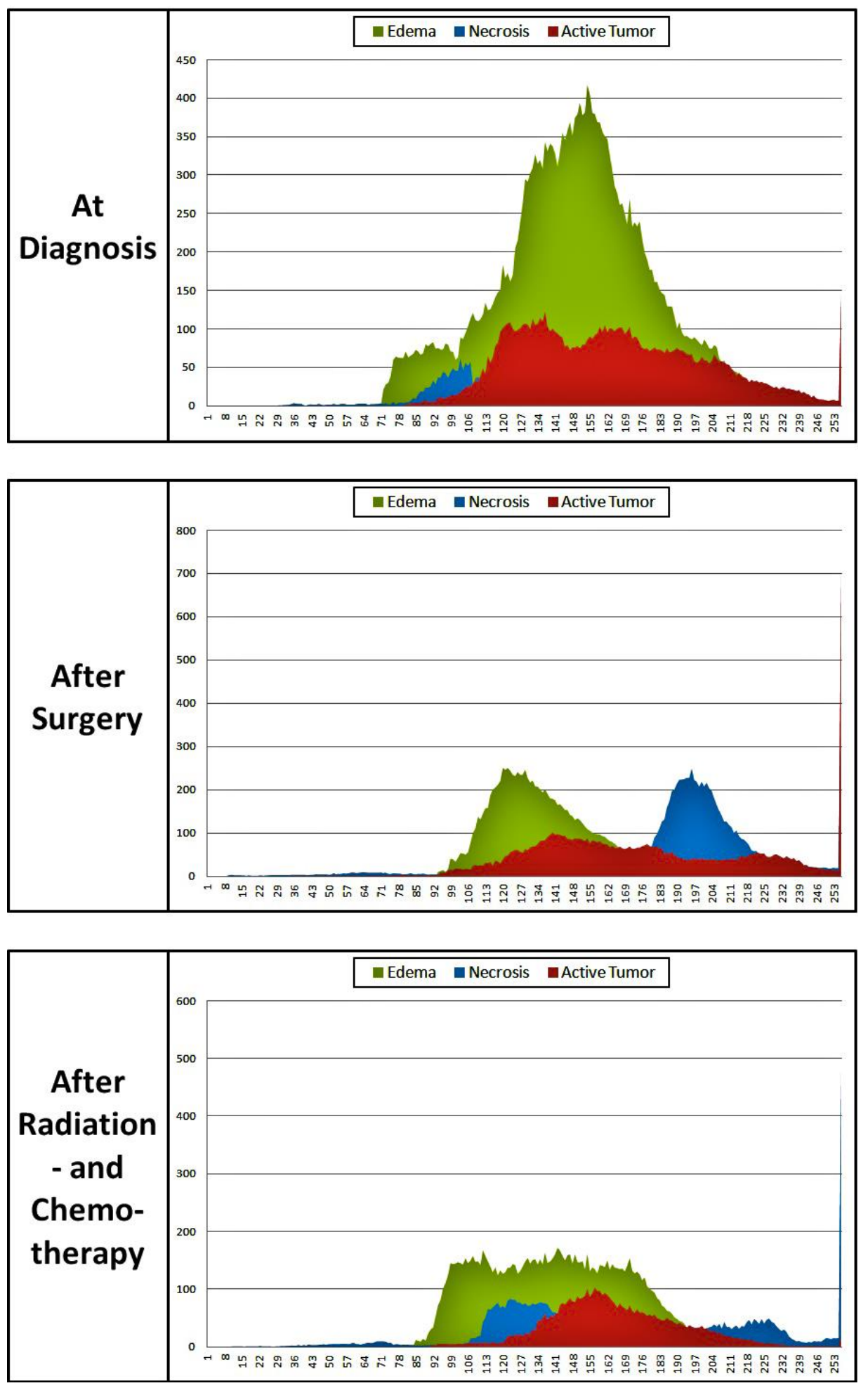

Figure 23: Mean Histogram from T2-Sequence during Follow-up 
Table XI: Results from mathematical Analysis of the mean Histograms from T2-Sequence during Follow-up

\begin{tabular}{|c|c|c|c|c|c|c|}
\hline \multirow{8}{*}{$\begin{array}{c}\text { At } \\
\text { Diagnosis }\end{array}$} & & Tumor & Necrosis & Edema & Whole Lesion & CSF \\
\hline & Volume of 1 Pixel $\left(\mathrm{mm}^{3}\right)$ & 4,12 & 4,12 & 4,12 & 4,12 & 4,12 \\
\hline & Total Pixel-Count & 9863 & 1393 & 25917 & 37173 & 15363 \\
\hline & Total Volume $\left(\mathrm{mm}^{3}\right)$ & 40.623 & 5.737 & 106.744 & 153.104 & 63.277 \\
\hline & Maximum Pixel-Count & 150 & 62 & 418 & 504 & 815 \\
\hline & Signalintensity-Peak & 255 & 102 & 153 & 153 & 255 \\
\hline & Mean Signalintesity & 163,1 & 102,0 & 148,8 & 150,9 & 174,5 \\
\hline & Standardization to CSF & 0,93 & 0,58 & 0,85 & 0,86 & 1,00 \\
\hline
\end{tabular}

\begin{tabular}{|c|c|c|c|c|c|c|}
\hline & & Tumor & Necrosis & Edema & Whole Lesion & CSF \\
\hline & Volume of 1 Pixel $\left(\mathrm{mm}^{3}\right)$ & 4,27 & 4,27 & 4,27 & 4,27 & 4,27 \\
\hline & Total Pixel-Count & 8830 & 9318 & 11465 & 29613 & 16197 \\
\hline After & Total Volume $\left(\mathrm{mm}^{3}\right)$ & 37.688 & 39.768 & 48.931 & 126.387 & 69.128 \\
\hline \multirow{4}{*}{ Surgery } & Maximum Pixel-Count & 736 & 433 & 251 & 1190 & 226 \\
\hline & Signalintensity-Peak & 255 & 255 & 119 & 255 & 163 \\
\hline & Mean Signalintesity & 176,0 & 193,4 & 136,8 & 166,3 & 169,1 \\
\hline & Standardization to CSF & 1,04 & 1,14 & 0,81 & 0,98 & 1,00 \\
\hline
\end{tabular}

\begin{tabular}{|c|c|c|c|c|c|c|}
\hline \multirow{8}{*}{$\begin{array}{c}\text { After } \\
\text { Radiation- } \\
\text { and Chemo- } \\
\text { therapy }\end{array}$} & & Tumor & Necrosis & Edema & Whole Lesion & CSF \\
\hline & Volume of 1 Pixel $\left(\mathrm{mm}^{3}\right)$ & 3,90 & 3,90 & 3,90 & 3,90 & 3,90 \\
\hline & Total Pixel-Count & 5158 & 6535 & 13473 & 25167 & 15684 \\
\hline & Total Volume $\left(\mathrm{mm}^{3}\right)$ & 20.109 & 25.480 & 52.530 & 98.119 & 61.147 \\
\hline & Maximum Pixel-Count & 103 & 498 & 171 & 516 & 267 \\
\hline & Signalintensity-Peak & 156 & 255 & 141 & 255 & 204 \\
\hline & Mean Signalintesity & 164,0 & 165,3 & 140,2 & 151,6 & 179,3 \\
\hline & Standardization to CSF & 0,91 & 0,92 & 0,78 & 0,85 & 1,00 \\
\hline
\end{tabular}



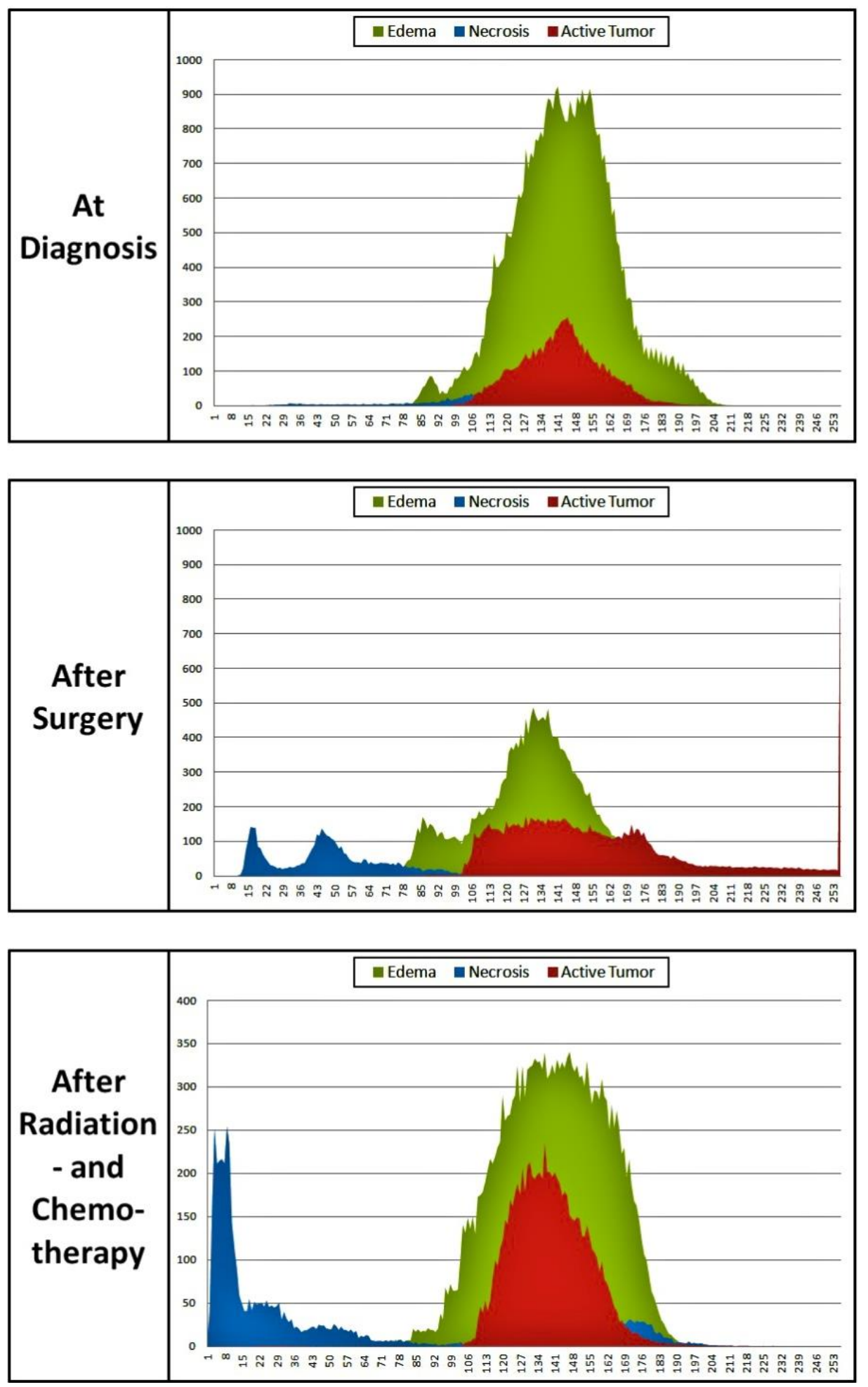

Figure 24: Mean Histogram from T2 Flair-Sequence during Follow-up 
Table XII: Results from mathematical Analysis of the mean Histograms from T2 Flair-Sequence during Follow-up

\begin{tabular}{|c|l|r|r|r|r|r|}
\hline & \multicolumn{1}{|c|}{ Tumor } & Necrosis & Edema & Whole Lesion & CSF \\
\cline { 2 - 7 } At & Volume of 1 Pixel $\left(\mathrm{mm}^{3}\right)$ & 4,43 & 4,43 & 4,43 & 4,43 & 4,43 \\
\cline { 2 - 7 } Diagnosis & 8783 & 1561 & 45886 & 56230 & 16388 \\
& Total Pixel-Count & $\mathbf{3 8 . 8 7 8}$ & $\mathbf{6 . 9 0 9}$ & $\mathbf{2 0 3 . 1 0 4}$ & $\mathbf{2 4 8 . 8 9 1}$ & $\mathbf{7 2 . 5 3 7}$ \\
& Total Volume (mm $\left.{ }^{3}\right)$ & 256 & 45 & 923 & 1148 & 438 \\
\cline { 2 - 7 } & Maximum Pixel-Count & 144 & 126 & 140 & 140 & 17 \\
& Signalintensity-Peak & 141,2 & 104,6 & 142,6 & 141,3 & 34,4 \\
Mean Signalintesity & $\mathbf{4 , 1 0}$ & $\mathbf{3 , 0 4}$ & $\mathbf{4 , 1 4}$ & $\mathbf{4 , 1 0}$ & $\mathbf{1 , 0 0}$ \\
\hline
\end{tabular}

\begin{tabular}{|c|l|r|r|r|r|r|}
\hline & \multicolumn{1}{|c|}{ Tumor } & Necrosis & Edema & Whole Lesion & CSF \\
\cline { 2 - 7 } & Volume of 1 Pixel $\left(\mathrm{mm}^{3}\right)$ & 4,47 & 4,47 & 4,47 & 4,47 & 4,47 \\
\cline { 2 - 7 } After & 13381 & 5298 & 22447 & 41126 & 13858 \\
Total Pixel-Count & Total Volume $\left(\mathbf{m m}^{3}\right)$ & $\mathbf{5 9 . 8 2 5}$ & $\mathbf{2 3 . 6 8 7}$ & $\mathbf{1 0 0 . 3 5 7}$ & $\mathbf{1 8 3 . 8 6 9}$ & $\mathbf{6 1 . 9 5 7}$ \\
& Maximum Pixel-Count & 921 & 142 & 487 & 927 & 450 \\
& Signalintensity-Peak & 255 & 15 & 130 & 255 & 17 \\
& Mean Signalintesity & 159,2 & 75,0 & 130,6 & 132,8 & 36,9 \\
& Standardization to CSF & $\mathbf{4 , 3 2}$ & $\mathbf{2 , 0 3}$ & $\mathbf{3 , 5 4}$ & $\mathbf{3 , 6 0}$ & $\mathbf{1 , 0 0}$ \\
\hline
\end{tabular}

\begin{tabular}{|c|c|c|c|c|c|c|}
\hline & & Tumor & Necrosis & Edema & Whole Lesion & CSF \\
\hline & Volume of 1 Pixel $\left(\mathrm{mm}^{3}\right)$ & 4,09 & 4,09 & 4,09 & 4,09 & 4,09 \\
\hline After & Total Pixel-Count & 8140 & 4874 & 20580 & 33593 & 14148 \\
\hline Radiation- & Total Volume $\left(\mathrm{mm}^{3}\right)$ & 33.275 & 19.923 & 84.128 & 137.326 & 57.837 \\
\hline and Chemo- & Maximum Pixel-Count & 235 & 255 & 340 & 574 & 469 \\
\hline therapy & Signalintensity-Peak & 136 & 8 & 146 & 136 & 17 \\
\hline & Mean Signalintesity & 137,9 & 49,1 & 139,4 & 126,0 & 36,5 \\
\hline & Standardization to CSF & 3,77 & 1,34 & 3,82 & 3,45 & 1,00 \\
\hline
\end{tabular}

\subsubsection{Non-Glioblastoma}

Patient G-8 represents one of the four patients in our study group that did not suffer from GBM (see Table II). In this patient a brain metastasis, deriving from bronchial cancer, could be diagnosed as the cause of disease. 


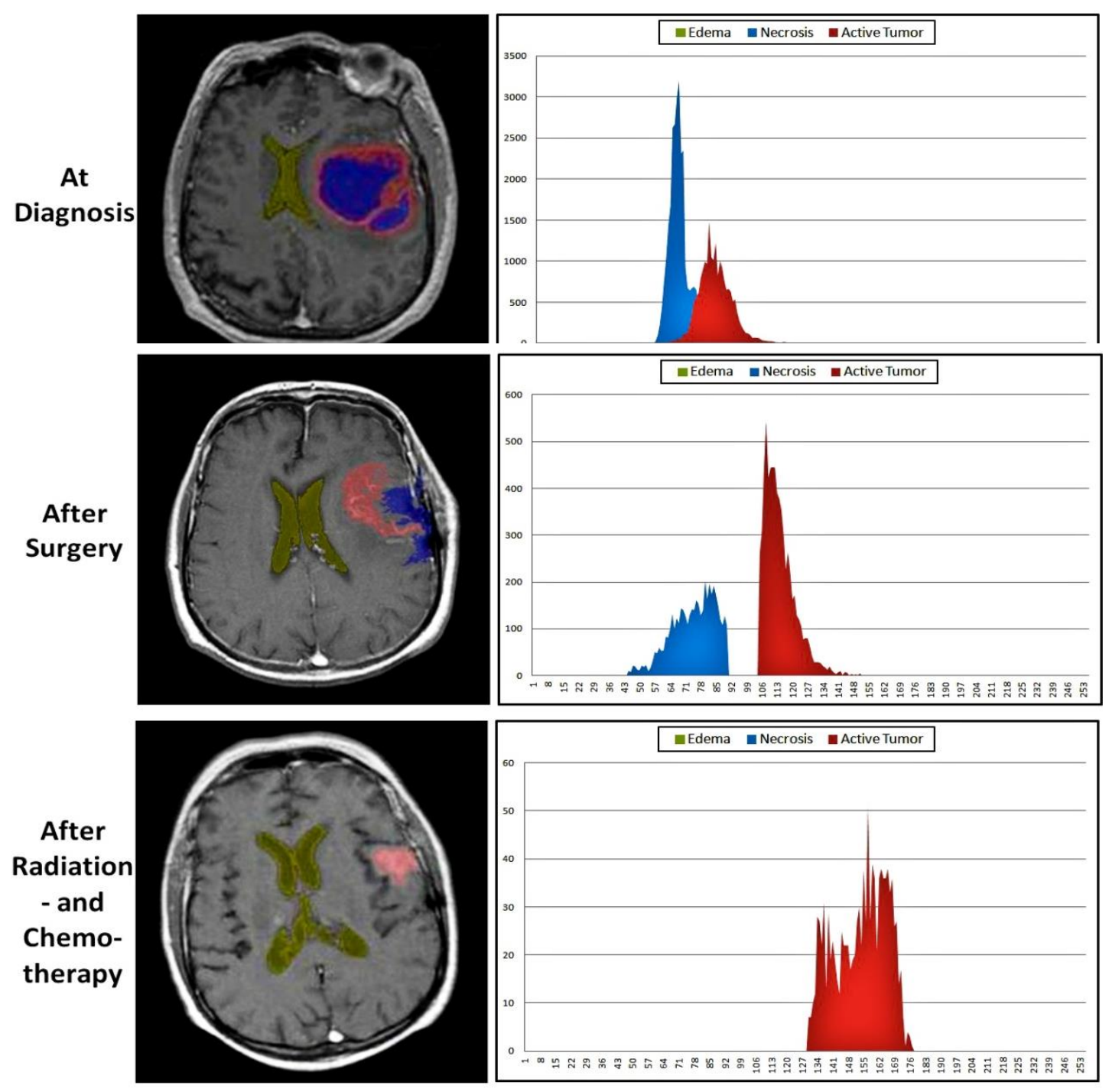

Figure 25: Follow-up of Patient G-8 in T1 with Gadolinium Contrast Enhancement Sequence 
Table XIII: Results from mathematical Analysis of Patient G-8 from T1 with Gadolinium Contrast Enhancement during Follow-up

\begin{tabular}{|c|c|c|c|c|c|c|}
\hline \multirow{8}{*}{$\begin{array}{c}\text { At } \\
\text { Diagnosis }\end{array}$} & & Tumor & Necrosis & Edema & Whole Lesion & CSF \\
\hline & Volume of 1 Pixel $\left(\mathrm{mm}^{3}\right)$ & 2,00 & 2,00 & & 2,00 & 2,00 \\
\hline & Total Pixel-Count & 19913 & 27780 & & 47693 & 4394 \\
\hline & Total Volume $\left(\mathrm{mm}^{3}\right)$ & 39.826 & 55.560 & & 95.386 & 8.788 \\
\hline & Maximum Pixel-Count & 1488 & 3195 & & 3249 & 347 \\
\hline & Signalintensity-Peak & 80 & 66 & & 66 & 42 \\
\hline & Mean Signalintesity & 83,0 & 66,1 & & 73,1 & 52,9 \\
\hline & Standardization to CSF & 1,57 & 1,25 & & 1,38 & 1,00 \\
\hline
\end{tabular}

\begin{tabular}{|c|c|c|c|c|c|c|}
\hline \multirow{8}{*}{$\begin{array}{c}\text { After } \\
\text { Surgery }\end{array}$} & & Tumor & Necrosis & Edema & Whole Lesion & CSF \\
\hline & Volume of 1 Pixel $\left(\mathrm{mm}^{3}\right)$ & 5,72 & 5,72 & & 5,72 & 5,72 \\
\hline & Total Pixel-Count & 6749 & 4435 & & 11184 & 7136 \\
\hline & Total Volume $\left(\mathrm{mm}^{3}\right)$ & 38.618 & 25.377 & & 63.995 & 40.833 \\
\hline & Maximum Pixel-Count & 544 & 203 & & 544 & 605 \\
\hline & Signalintensity-Peak & 107 & 79 & & 107 & 49 \\
\hline & Mean Signalintesity & 113,3 & 73,6 & & 97,5 & 54,9 \\
\hline & Standardization to CSF & 2,06 & 1,34 & & 1,78 & 1,00 \\
\hline
\end{tabular}

\begin{tabular}{|c|c|c|c|c|c|c|}
\hline \multirow{8}{*}{\begin{tabular}{|c} 
After \\
Radiation- \\
and Chemo- \\
therapy
\end{tabular}} & & Tumor & Necrosis & Edema & Whole Lesion & CSF \\
\hline & Volume of 1 Pixel $\left(\mathrm{mm}^{3}\right)$ & 2,89 & & & 2,89 & 2,89 \\
\hline & Total Pixel-Count & 1092 & & & 1092 & 3293 \\
\hline & Total Volume $\left(\mathrm{mm}^{3}\right)$ & 3.157 & & & 3.157 & 9.521 \\
\hline & Maximum Pixel-Count & 51 & & & 51 & 199 \\
\hline & Signalintensity-Peak & 156 & & & 156 & 51 \\
\hline & Mean Signalintesity & 152,9 & & & 152,9 & 57,1 \\
\hline & Standardization to CSF & 2,68 & & & 2,68 & 1,00 \\
\hline
\end{tabular}

\subsubsection{Summary Analysis - Overview}

Tables XIV-XVI give a clear overview about the three most meaningful aspects of our mathematical analysis, the SI-peak, the mean SI and the standardized mean $\mathrm{SI}$, calculated from all patients of this study. Emphasis is placed on the comparison between active tumor and necrosis in the different sequences and the summarized whole lesion. 
Table XIV: SI-Peak of all Sequences at different Time Points calculated from all Patients of this Series

\begin{tabular}{|c|c|c|c|c|c|}
\hline \multicolumn{2}{|c|}{ Signalintensity-Peak } & T1 & $\mathrm{T} 1+\mathrm{Gd}$ & T2 & T2 Flair \\
\hline \multirow{2}{*}{ At Diagnosis } & Tumor & 85 & 88 & 255 & 144 \\
\hline & Necrosis & 33 & 68 & 102 & 126 \\
\hline \multirow{2}{*}{ After Surgery } & Tumor & 127 & 127 & 255 & 255 \\
\hline & Necrosis & 46 & 47 & 255 & 15 \\
\hline \multirow{2}{*}{$\begin{array}{c}\text { After } \\
\text { Radiation- } \\
\text { and Chemo- }\end{array}$} & Tumor & 76 & 119 & 156 & 136 \\
\hline & Necrosis & 28 & 24 & 255 & 8 \\
\hline \multicolumn{6}{|c|}{ Whole Lesion } \\
\hline \multicolumn{2}{|c|}{ At Diagnosis } & 79 & 69 & 153 & 140 \\
\hline \multicolumn{2}{|c|}{ After Surgery } & 46 & 47 & 255 & 255 \\
\hline \multicolumn{2}{|c|}{ After Radiation- and Chemoth. } & 79 & 97 & 255 & 136 \\
\hline
\end{tabular}

Table XV: Mean Signalintensity of all Sequences at different Time Points calculated from all Patients of this Series

\begin{tabular}{|c|c|c|c|c|c|}
\hline \multicolumn{2}{|c|}{ Mean Signalintensity } & \multirow{2}{*}{$\begin{array}{c}\mathrm{T} 1 \\
82,0\end{array}$} & \multirow{2}{*}{$\frac{\mathrm{T} 1+\mathrm{Gd}}{116,2}$} & \multirow{2}{*}{$\frac{\mathrm{T} 2}{163,1}$} & \multirow{2}{*}{$\begin{array}{r}\text { T2 Flair } \\
141,2\end{array}$} \\
\hline & Tumor & & & & \\
\hline & Necrosis & 51,7 & 67,6 & 102,0 & 104,6 \\
\hline \multirow{2}{*}{ After Surgery } & Tumor & 121,3 & 121,4 & 176,0 & 159,2 \\
\hline & Necrosis & 54,8 & 63,1 & 193,4 & 75,0 \\
\hline \multirow{2}{*}{$\begin{array}{c}\text { After } \\
\text { Radiation- } \\
\text { and Chemo- }\end{array}$} & Tumor & 90,0 & 125,5 & 164,0 & 137,9 \\
\hline & Necrosis & 43,2 & 46,3 & 165,3 & 49,1 \\
\hline \multicolumn{6}{|c|}{ Whole Lesion } \\
\hline \multicolumn{2}{|c|}{ At Diagnosis } & 70,8 & 80,0 & 150,9 & 141,3 \\
\hline \multicolumn{2}{|c|}{ After Surgery } & 80,5 & 84,2 & 166,3 & 132,8 \\
\hline \multicolumn{2}{|c|}{ After Radiation- and Chemoth. } & 75,8 & 82,4 & 151,6 & 126,0 \\
\hline
\end{tabular}


Table XVI: Standardized mean SI of all Sequences at different Time Points calculated from all Patients of this Series

\begin{tabular}{|c|l|cccc|}
\hline \multicolumn{2}{|c|}{ Standardized Signalintensity } & T1 & T1 + Gd & T2 & T2 Flair \\
\hline \multirow{2}{*}{ At Diagnosis } & Tumor & 1,57 & 2,26 & 0,93 & 4,10 \\
& Necrosis & 0,99 & 1,32 & 0,58 & 3,04 \\
\hline \multirow{2}{*}{ After Surgery } & Tumor & 1,98 & 2,29 & 1,04 & 4,32 \\
& Necrosis & 0,89 & 1,19 & 1,14 & 2,03 \\
\hline $\begin{array}{c}\text { After } \\
\text { Radiation- } \\
\text { and Chemo- }\end{array}$ & Tumor & 1,59 & 2,30 & 0,91 & 3,77 \\
\hline \multicolumn{2}{|c|}{ Whole Lesion } & 0,76 & 0,85 & 0,92 & 1,34 \\
\hline \multicolumn{2}{|c|}{ At Diagnosis } & 1,36 & 1,56 & 0,86 & 4,10 \\
\hline \multicolumn{2}{|c|}{ After Surgery } & 1,32 & 1,59 & 0,98 & 3,60 \\
\hline After Radiation- and Chemoth. & 1,34 & 1,51 & 0,85 & 3,45 \\
\hline
\end{tabular}

\subsubsection{Volume Changes during Follow-up}

By multiplying the total pixel-counts of the different lesions with its particular pixelsize, we were able to calculate the exact volume of each area that we have rendered. Figure 26 exemplary shows the course of disease of a single patient (G-25), by illustrating a volumetric development for the whole lesion, as well as the different subunits of tumorous tissues during the different time points and for all MR-Sequences.

The second image in this section, Figure 27, depicts the mean volume changes during therapy in all MR-Sequences, calculated from all patients of the study group (except the non-glioblastomas). As the previous image does, it also compares the whole lesion with its three subunits active tumor, necrosis and edema. 


\subsubsection{Single Patient}
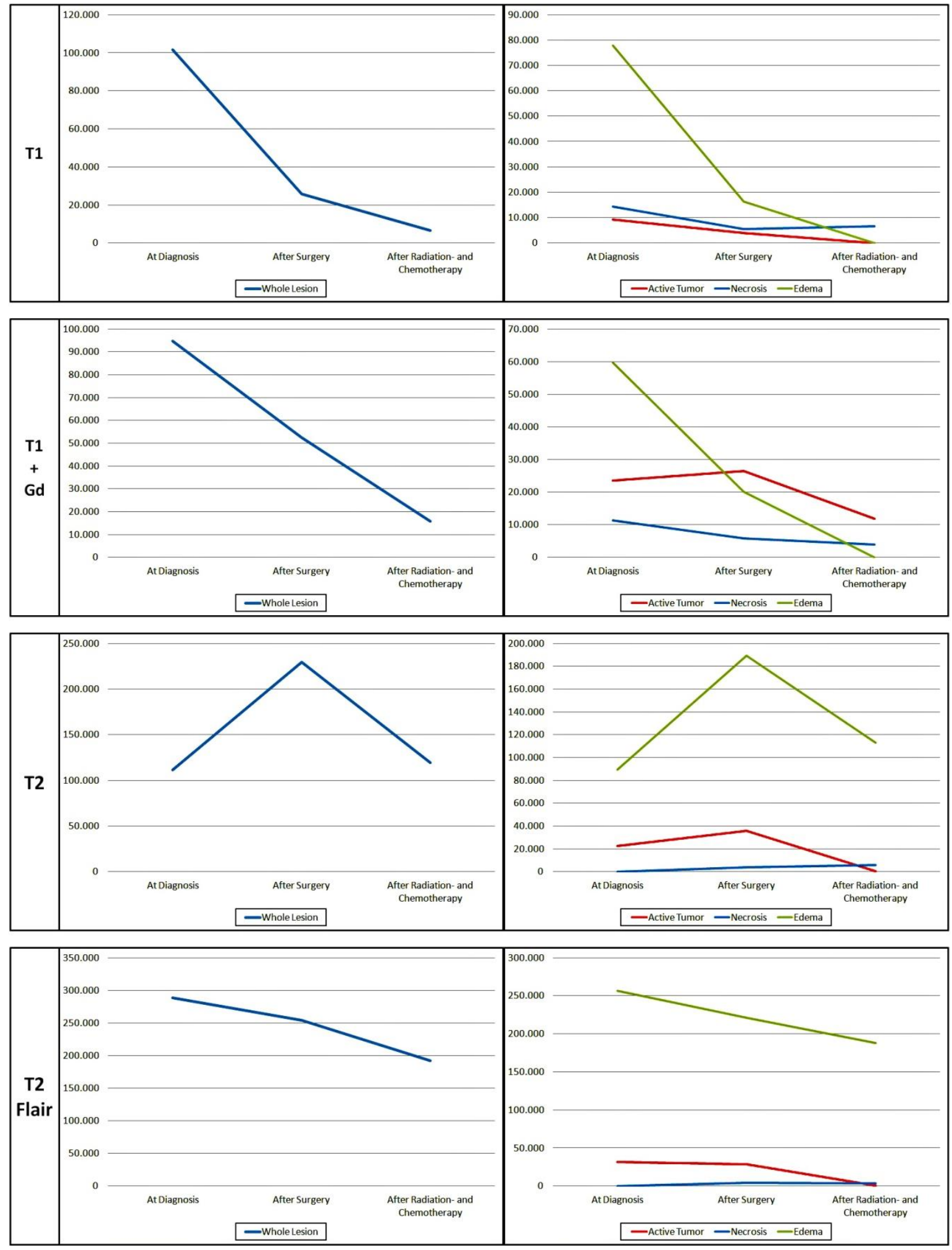

Figure 26: Volume Changes during Follow-up in Patient G-25 for all Sequences. Comparison of the whole Lesion to the three Subunits (Active Tumor, Necrosis and Edema). 


\subsubsection{Summary}
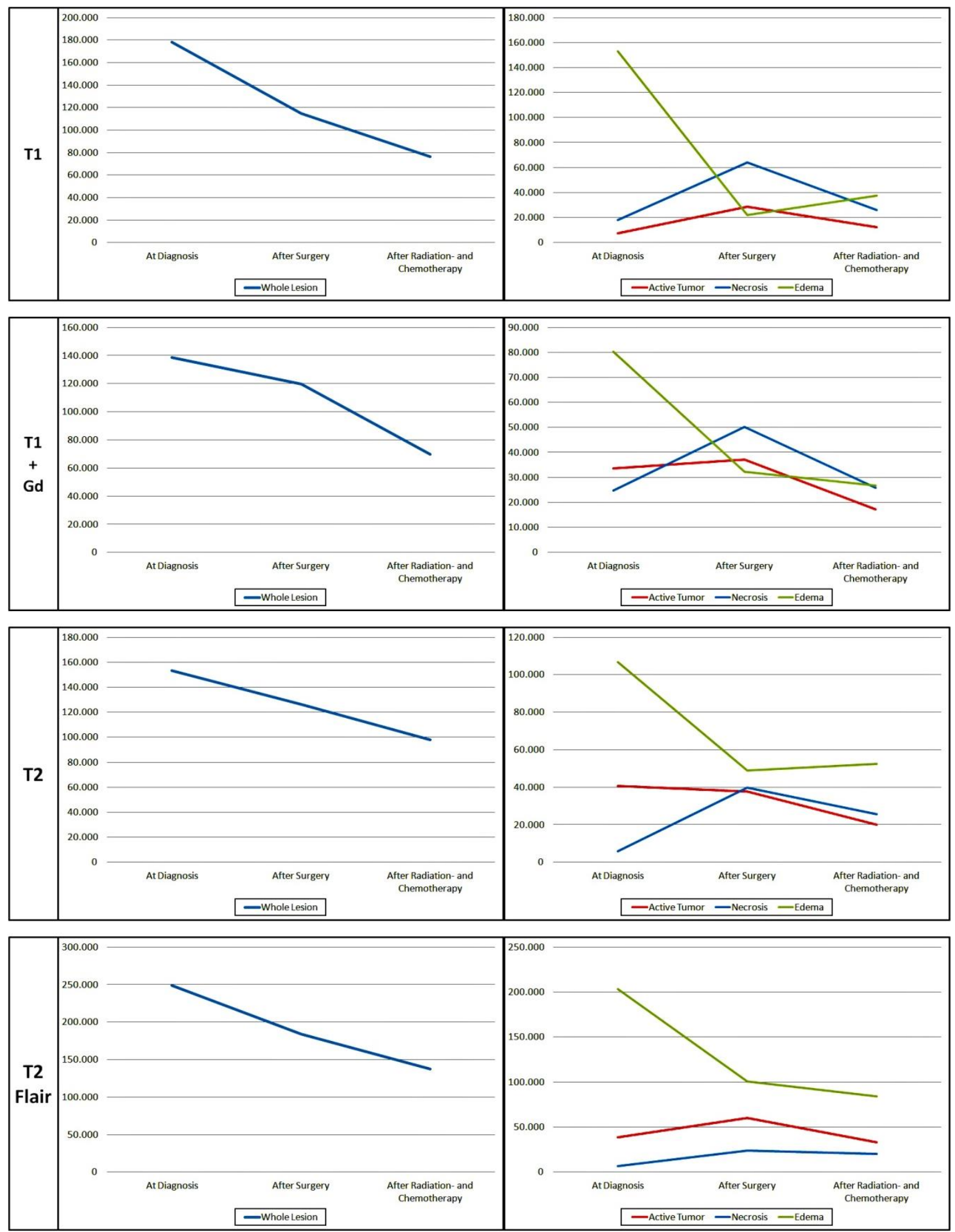

Figure 27: Volume Changes during Follow-up in the Summary, for all Sequences. Comparison of the whole Lesion to the three Subunits (Active Tumor, Necrosis and Edema). 


\subsubsection{Assessment of Progression and Response}

This section illustrates four different possible courses of disease on the basis of the individual volume development during treatment and follow-up. Figure 28 shows how the volume of the whole lesion and the different pathologic subunits develops in a patient that clearly suffers from real tumor recurrence, after receiving surgery, RT and chemotherapy (G-17). In Figure 29 the volume development of a patient that shows pseudoprogression, after receiving surgery, RT and chemotherapy (G-12), is depicted. Figure $\mathbf{3 0}$ displays the course of disease from a patient with stable disease (G-7), whereas the response shown in Figure $\mathbf{3 1}$ is rather questionable due to the contradictory volume development of the three subunits (G-23). 


\subsubsection{Real Progression in T1 with Gadolinium Contrast Enhancement}

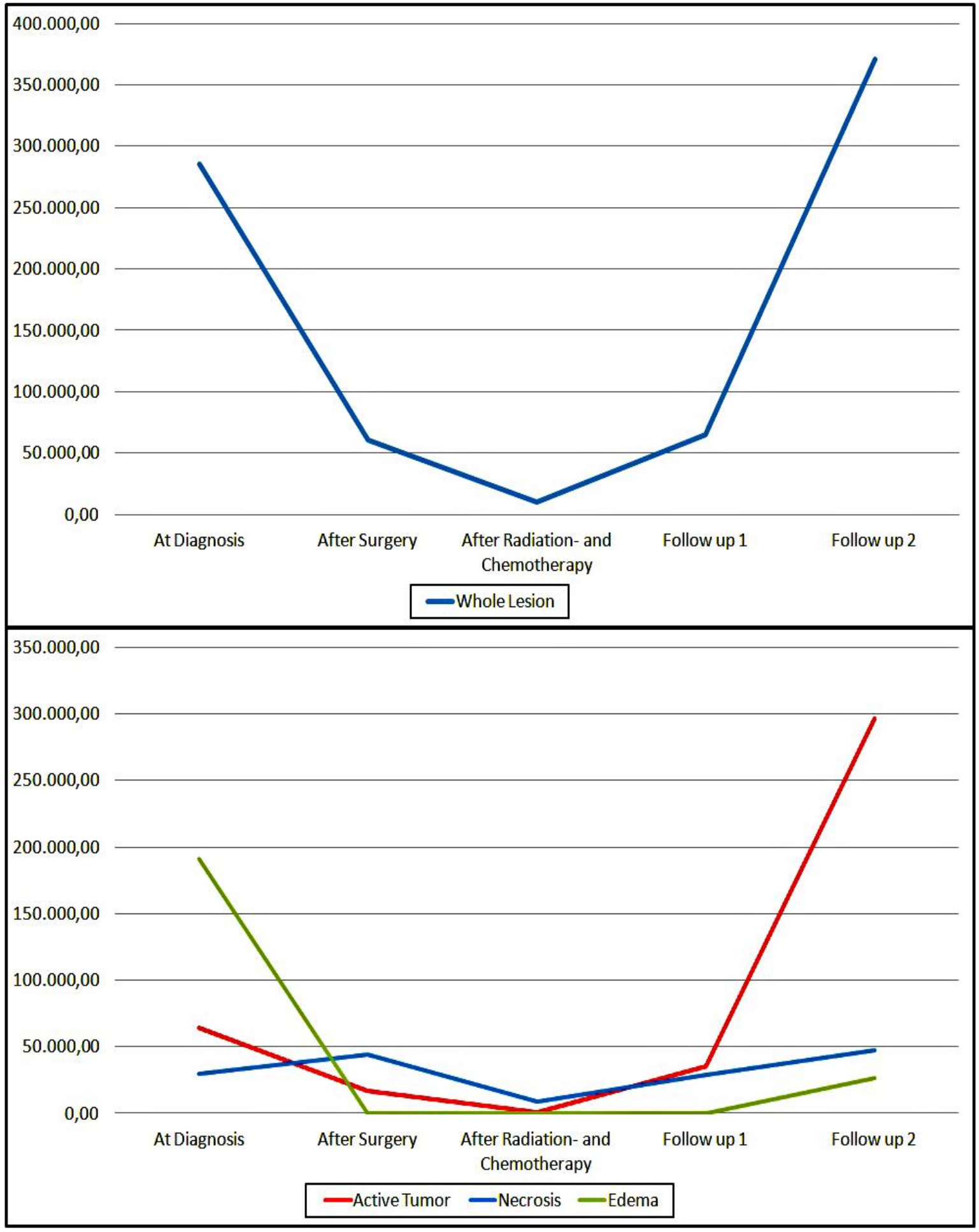

Figure 28: Volume Development during different Time points in a Patient that suffers from real Tumor Progression after receiving Surgery and Radiation- and Chemotherapy (G-17) 


\subsubsection{Pseudoprogression in T1 with Gadolinium Contrast Enhancement}

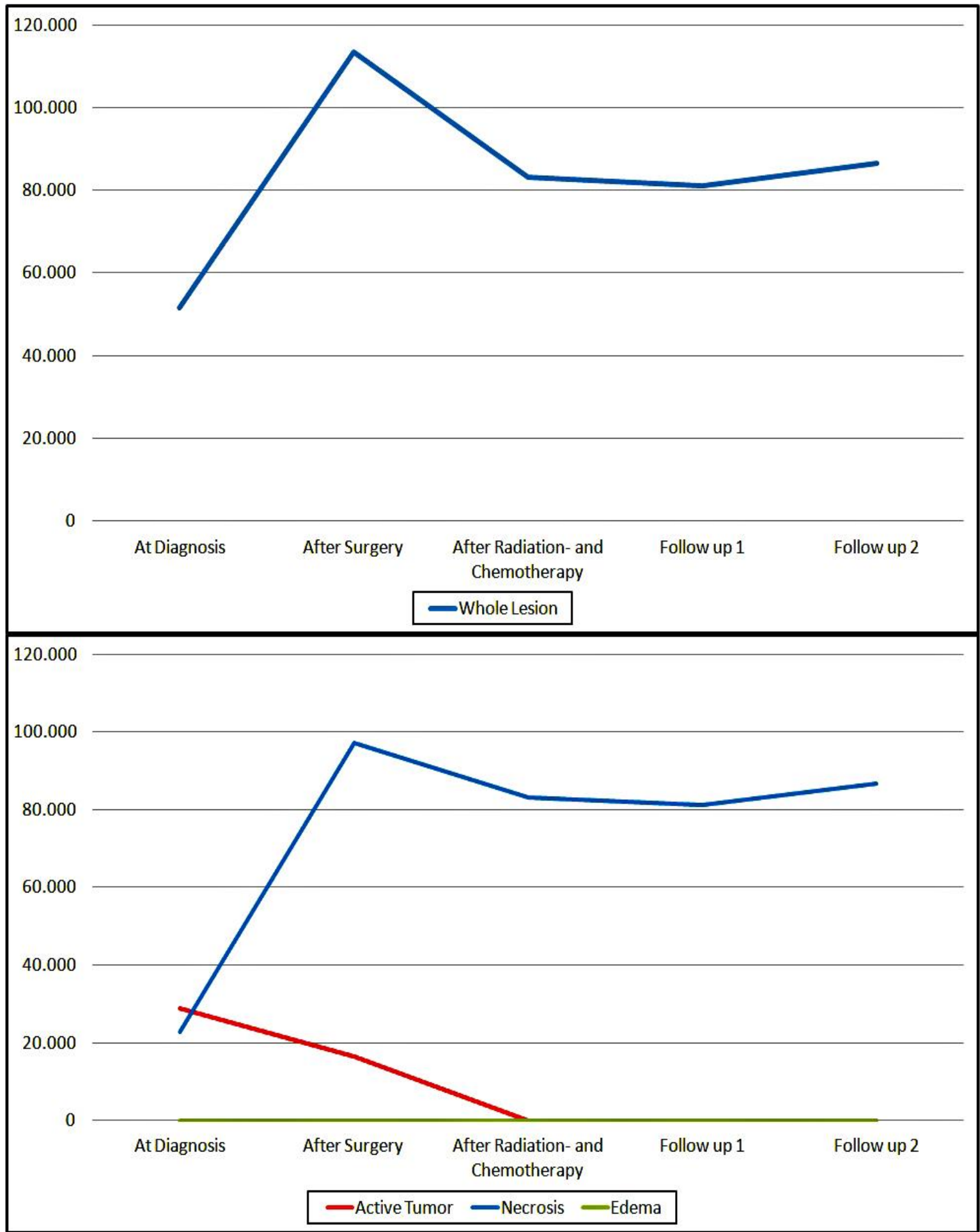

Figure 29: Volume Development during different Time Points in a Patient that shows questionable Progression after receiving Surgery and Radiation- and Chemotherapy (G-12) 


\subsubsection{Stable Disease in T1 with Gadolinium Contrast Enhancement}

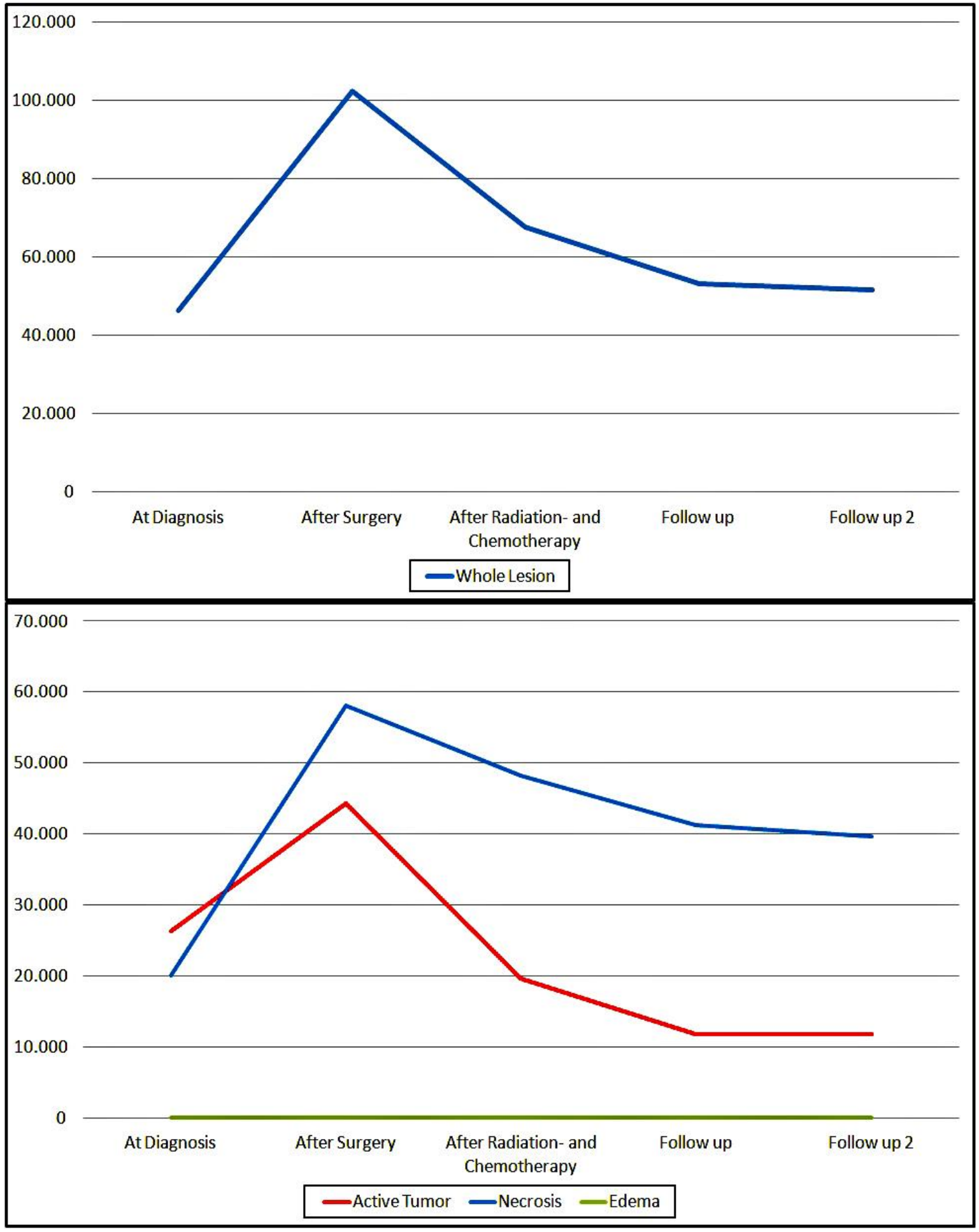

Figure 30: Volume Development during different Time Points in a Patient that shows stable Disease after receiving Surgery and Radiation- and Chemotherapy (G-7) 


\subsubsection{Questionable response in T1 with Gadolinium Contrast Enhancement}

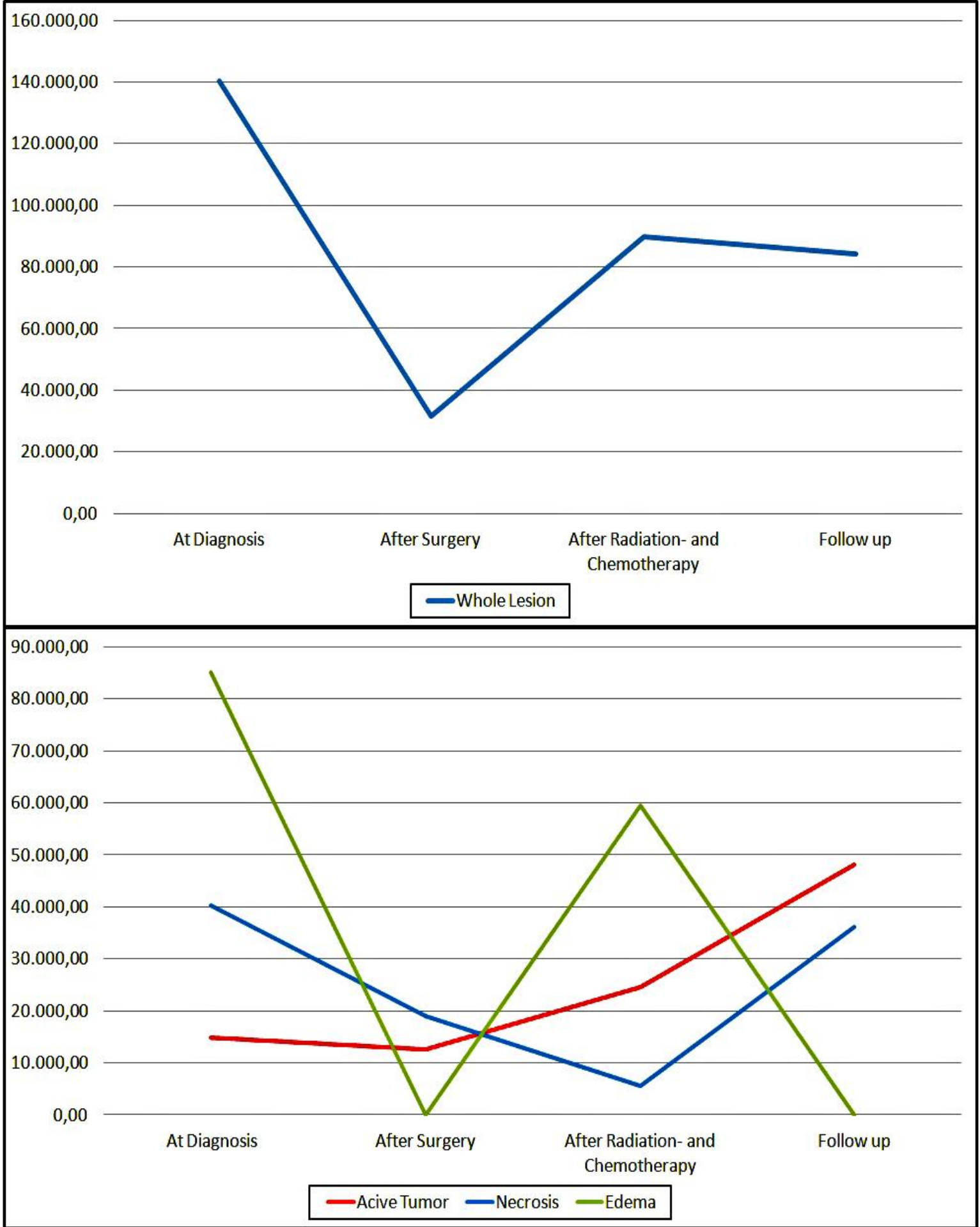

Figure 31: Volume Development during different Time Points in a Patient that shows questionable Response after receiving Surgery and Radiation- and Chemotherapy (G-23) 


\section{Discussion}

\subsection{Evaluation of Software and Segmentation Methods}

DoctorEye represents an user-friendly segmentation software which allowed us to perform relatively fast annotations of different data sets at the same time, with a high level of accuracy. Our lively cooperation with the developers of FORTH enabled us to impact the platform's updates and designing. Over the two years of our research project our suggestions and input for improvement primarily focused on the software's practical handling and its applicability in the clinical routine. Additionally we came up with the idea to create histograms based on the annotated areas, a suggestion that was immediately implemented into the software by the developers.

During the last two decades there have been multiple attempts to analyze medical images using modern information technologies. Currently there are several other existing image-annotation platforms, including VANO [109], CMAS [90], Hybrid System [99], Annotor [2], Amiravis [1], Itk-SNAP [4] and many more [3, 5]. Even if these various tools have been developed to speed up the process of data analysis for different biomedical applications, still most of the open access solutions require extensive engineering skills to understand their sophisticated user interface. In contrast to DoctorEye their whole design rarely takes the actual clinical user's needs or the specific features required in the clinical setting and the goal to derive qualitative and quantitative diagnostic information into account [126]. DoctorEye on the other hand was found to be an impressively user-friendly segmentation software that allowed us to simultaneously perform fast annotations of different data sets, with a high level of accuracy. After using the software over a large number of datasets, in our hands, the platform's environment appeared very intuitive and easy to handle. In fact, our experience over the complete study period confirmed our initial impression that only basic knowledge of annotation/simulation processes is required to start up using this system effectively. 
The main advantages of DoctorEye in comparison to already available imageprocessing tools have already been pointed out by Skounakis et al. and include the following $[49,126]$ :

- Open access software with free distribution for testing and no need for registration or activation.

- Easy to use, due to the platform's design as a GUI environment with standard menus and buttons. Its interface is flexible and can be easily customized to different viewing setups by adjusting the width and height of the thumbnail and the main window by sliding the window separator bar. In consequence, the user can actively alter the number of the thumbnails visible on the screen as well as the size of the selected image. The resolution and size of the user's screen are also taken into account for ensuring an optimal representation.

- Fast and accurate delineation of tumors in large number of 3D tomographic datasets, especially in areas with complicated shapes, facilitated by manual and semiautomatic segmentation techniques combined with integrated correction tools. The fast operation-time is realizable due to the fact that the applied algorithms are fully integrated in the platform and run "on the fly" and not as calls to external programs.

- The ability to set multiple labels that allow the user to annotate and manage multiple areas of interest in each selected slide at the same session.

- The user is permitted to view the effects of the features that are being implemented in the selected medical image while experimenting with various parameters.

- More importantly the clinical driven modular design and functionality, ensuring that the software has the potential to be implemented in routine clinical practice. 
- Additionally to "standard" features like annotation/segmentation and 3D visualization the flexible architecture of DoctorEye provides the opportunity for easy incorporation of more specific and sophisticated plug-ins that can empower the clinician to use modern technologies for enhanced diagnosis and therapy design. An exemplary plug-in enables the user to do computational "in-silico" models of cancer growth and simulation algorithms of therapy response in order to provide a future integrated platform for modeling assisted therapy decision making.

As a tool for predictive oncology research and clinical work DoctorEye can allow cancer modellers to easily plug in their models for cancer growth and therapy simulation. Moreover, besides being a tool for analyzing cancer imaging data, at the same time it could also serve as a validation environment for clinicians, where the simulation predictions can be compared with the actual therapy outcome, in order to achieve a global optimization of the modeling modules.

The final assessment of the methods engaged indicates that the "Magic Wand" method provides great ease of use and is more applicable to medical images, especially when the gray level profile of the tumor is very homogenous and there is enough contrast to the surrounding tissues. In more problematic settings the software offers sophisticated solutions. If the regions of interest exhibit a high structural complexity, the "Zoom In" mode can facilitate the rendering enormously by magnifying the investigated area. If the result obtained by only using the Magic Wand is not satisfactory, manual refinement can be performed by engaging the correction tools "pencil and eraser", to achieve a most precise result, as illustrated in Figure 11 and 12.

The accuracy of this technology was confirmed by the well-recognized expert in neuroradiology Prof. Reith from the University of the Saarland. Our results regarding the delineation of different pathologic areas in different MRI scans were in total accordance with the expert's assessment, clearly demonstrating that a higher accuracy cannot be achieved by any other segmentation method. However, it has to be acknowledged that even with DoctorEye the evaluation of very 
complex structured lesions with a highly heterogeneous distribution of SI still requires a high amount of patience and time. An untrained user most likely requires a processing time of more than 15 minutes (see results 4.1.1) for one sequence, respectively 20 slices. Such time consuming evaluations would prove to be still very inexpedient for a clinician's tight scheduled daily workflow. Although the time necessary to get the software ready-to-use, to up-load large datasets und to evaluate and safe the results is almost invincible fast, the use of the "Magic Wand" algorithm is yet too time-consuming and not practicable to be utilized in clinical daily routine.

Apart from still being clinically unsuitable due to the time consuming evaluation processes, manual- and largely semiautomatic-segmentation techniques have an additional drawback. According to Weltens et al. inter-observer variability in brain tumor delineation with different physician specialties performing contours in both CT alone and CT with MRI ranged between 9-32\% [155]. Mazzara et al. showed that the intra- and inter-rater variability performing manual segmentations on MRI scans of patients with gliomas varies significantly, being reported to be approximately $20 \% \pm 16 \%$ and $28 \% \pm 12 \%$, respectively [98] .

Following our experience with DoctorEye a more expeditious technique has to be implemented to make the method applicable for the routine medical environment as well as to reduce operator-dependency during evaluation. Either an automatic or an advanced semi-automatic methodology probably can achieve this. Kaus et al. reported in 2001 that their technique of automated tumor segmentation in MR images performed essentially faster than the compared technique of manual rendering, which is in principle comparable to our software [74].

Such an automatic segmentation technique should on the one hand provide the option to accurately delineate complex pathologic areas and on the other hand speed up the operating time in treatment planning, by keeping the need for user interaction as low as possible. Additionally, the measurement protocol should be standardized and reproducible, so that it can be applied in any clinical environment independent from the clinician's prior training. 
The publication mentioned above by Mazzara et al. reported about the evaluation of two different segmentation methods, an automatic and a semiautomatic one. Validation was performed by the comparison to manual segmentation [98]. The fact that all the study subjects suffered from primary brain cancer (glioma) but not specifically from GBM slightly reduces the comparability of these results with our observations. This is related to the structural complexity often found with GBM in medical images, which frequently affects accuracy and operation-time. Mazzara et al. evaluated the k-nearest neighbor ( $k N N)$ system, as a representative of operator-assisted semiautomatic segmentation, and the knowledge-guided (KG) method, as a representative of fully automated tumor volume measurement. While the kNN system requires the user to select training data from each MRI slice, the KG method does not need any user input and has therefore in principle no variability in its output. Both methods have been successfully studied and clinically used for improved accuracy of tumor volume measurements since almost 25 years $[40,145]$. From the three methods investigated the KG method required the least operation time, in average 8,2 minutes per patient, followed by the kNN system, requiring 20 minutes per patient. The manual method proved to be the slowest and was recorded to consume in average 30 minutes for the evaluation of an individual patient. Relevant for our studies on GBM is the fact that in comparison to the kNN method the KG method performed poorly for glioma cases, which showed Gdenhancement with non-enhancing cystic necrotic centers. This is considered to be due to the need of user input for the initial selection of training pixel data in the kNN method. As glioblastoma often exhibit cystic necrotic centers, the technique described by Mazzara appears to be inappropriate to provide valid volume data. Also the KG method does not segment non-enhancing tumors. In conclusion the two methods described by Mazzara lack accuracy compared to the manual assessment of radiation oncologists, but are superior in terms of reproducibility and processing time.

Kumar M. et al proposed in 2011 another automatic seeded region growing method to segment brain tumors in 2 D MR images [82]. However, while their results indicate that fast and accurate segmentation by automatically finding the 
seed points for the region growing method can be achieved, the tumors analyzed in this study appear to be structurally less complex as the GBMs analyzed by us. Before this approach is not validated in lesions comparable to glioblastoma, the value of this technique for our purposes cannot be judged.

In 2011 Mohd Saad et al. applied an automated region growing technique on Diffusion-weighted MRI, focusing not only on brain tumors but also on acute infarction, haemorrhage and abscesses [102]. First, region splitting and merging was performed to subdivide the image with preservation of the pathological area. Second, the histogram threshold was used to find the optimal intensity threshold value, which is the prerequisite for the automatic selection of the initial seed-point. The results of their study showed that their automatic solution of region growing provided comparable results with the semiautomatic segmentation in terms of accuracy. However, their sample-set again was highly heterogeneous, consisting not only of glioblastoma but also including 3 cases of abscesses, 4 haemorrhage, 11 acute infarction and 2 tumors with unmentioned histology. As discussed with the study before this method needs to be validated on GBM cases before its value in GBM diagnostics can be assessed reliably. Choubey and Agrawal published their results in 2012, also describing an automatic segmentation method based on the region growing, the so called "random walk movement". Once more in this approach the seed point is automatically calculated, following a skull removal to prevent falsified seed point selection. As proposed, this method ought to be effective in 2 dimensional MRI, but the accuracy depends on the lesion's shape and size (very small tumors fail to be detected) [39].

Another frequently used segmentation method is the so called "fuzzy connectivity" or "fuzzy connectedness algorithm". Through the fuzzy process the fuzzy connectedness algorithm joins related pixels in the investigated region despite inhomogeneity of signal intensities or gradient variations [61]. This concept of fuzziness was firstly introduced by Rosenfeld in 1970, as affirmed by Harati et al. $[61,120]$. Just as with Region-Growing, the selection of the initial seed point in this technique is a substantial and time-consuming task and inevitably requires an 
operator. Therefore Harati V. et al. developed a fully automatic segmentation procedure with the purpose to eliminate user interactions in the seed point selection and to improve the performance of the general fuzzy connectedness [61]. To automatically select the seed point and to execute the tumor segmentation algorithm in average an operation time of $30 \mathrm{~s}$ and 2 min was consumed [61]. The authors claim that this procedure does not go at the expanse of accuracy. To confirm this assertion, they compared their method with the general fuzzy connectedness, represented in 1996 by Udupa and Samarasekera [142]. Almost all recent approaches in the field of fuzzy connectedness are based on the principles of that specific algorithm. The criteria values revealed that the performance of the proposed method is superior to that of general fuzzy connectedness in all investigated cases. In a more topical study from 2006, Pednekar et al. proposed one of the state-of-the-art approaches related to the improved generalized fuzzy connectedness [108]. An important drawback of this study in comparison to the study mentioned before lies in the small amount of data that has been investigated and the fact that seed points were selected manually, whereas in the aforementioned method seed points were selected automatically. One advantage, however, is that the method, according to Pednekar et al., can be applied to different types of radiologic images such as MR, CT and infrared data [108]. This is attributable to the dynamic tuning of weights of affinity components as required in the fuzzy connectedness algorithm, whereas in the method proposed by Harati V. et al. constant optimized weights are used, which is only applicable to brain MR images [61].

Another recently proposed approach for fully automatic brain tissue segmentation was published by Bauer S. et al. in 2011. This group is part of the ContraCancrum project and obtained images of 10 study patients from the ContraCancrum brain tumor database [22]. In this study the combination of the Support Vector machine classification (SVM) using multispectral intensities and textures with subsequent hierarchical regularization based on Conditional Random Fields showed promising results. According to the authors, there is a strong dependency of most voxel labels on their neighbors, a statement that is in disagreement with the assumption 
that the data in MR images are independent and identically distributed (iid). Bauer and coworkers engaged the Conditional Random Field (CRF) method to respect these spatial relationships. The way of applying the CRF was inspired by a study performed by Lee et al. in 2008 [88]. As in our study, Bauer et al. subdivided the tumor area hierarchically into necrotic, active and edematous tumor portions, besides generally distinguishing between normal healthy and tumor region. By using the Insight Toolkit for Segmentation and Registration (ITK) they were able to incorporate the preprocessing completely with the SVM classification and CRF regularization components [70]. Quantitative evaluation of the tumor tissue segmentation again was performed with the ground truth defined by manual segmentation, using the dice similarity coefficient. As a result it was shown that this coefficient is evidently a worse measure when no hierarchical regularization is applied. The operation time for the segmentation algorithm mainly depends on the size of the image dataset and on the complexity of the SVM optimization. It performs obviously faster than our approach, taking between 20 and 120 seconds on a single CPU (Central Processing Unit) running at 2,33 GHz. However, it has to be taken into account that preprocessing was not included in this estimation. Compared to other studies, like Corso et al [42] and Wels et al. [154], dice similarity coefficients for the gross tumor volume are in a similar range, but different data-sets were used. Additionally, although in this approach differentiation into tumor and healthy subsets was performed, the operation time was shorter. In terms of accuracy the results are comparable to manual segmentation, with the advantage of excluding biased subjectively of the operators in longitudinal studies. Since differentiating the tumor into its subunits causes additional difficulties, the dice similarity coefficients for the subunits are lower than for the gross tumor volume. In conclusion, this new approach has great potential, as its operation time appears to be acceptable for the clinical workflow and by including the CRF based hierarchical regularization, improved conformity with the ground truth was achieved.

Finally, the incorporated adaptive active contour algorithm, developed by ICS group of FORTH, has shown promising results in closely following clinical 
annotations, as demonstrated by Farmaki et al. [50]. As described by Skounakis et al., this method is non intuitive, implying that the user might find it difficult to interpret the physical meaning of the related parameters and to predict the optimal values to be selected and adjusted individually for every tumor case [127].

\subsection{Assessment of Progression and Response}

Since 1990 the MacDonald criteria [96], based on measurable changes in contrast-enhancing lesions on MR scans, have been the standard approach for measuring response or progression in patients with malignant glial tumors. In 2010 the RANO Working Group suggested new criteria [159] that indicated the limitations of the above mentioned approach and added additional aspects. Nevertheless, neither a concrete assessment of changes in T2-/Flair-sequence is required nor is a volumetric measurement of the contrast enhanced lesions suggested. The dependence of the contrast enhancement in the T1 sequence on many parameters is another source of inaccuracy of these criteria [151]. In consequence it has to be acknowledged that the currently available guidelines are still insufficient and lack in accuracy [24]. Therefore, the process of analyzing the follow-up of individual patients with malignant gliomas requires more research and improvement of diagnostic procedures to achieve better understanding and facilitate individualized treatment of this currently still fulminant and lethal disease.

The lack of an established concept to follow tumor development and progression inspired us to search for an innovative approach of gaining tumor specific characteristics from routinely acquired MR images. The current routine to measure tumor size only by the product of the longest diameter and its longest perpendicular diameter in a single slice seems to be highly inappropriate, as glioblastoma tend to be very irregularly shaped. For that reason we decided to also incorporate a three dimensional volume measurement of the different tumorous tissues into our study. Furthermore, we applied our method not only on T1 Gd-MRI scans, but also on the other routinely acquired sequences, particularly T1 without contrast, T2 and T2 Flair. As mentioned above, these sequences do 
not play any role in the official criteria and clinically just serve for visual interpretation performed by neuroradiologists. However, it was our goal to extract as much information from standardly available images as possible. Our findings support the observation that by measuring volumes and segmenting the tumor in different sequences active tumor-tissue, necrotic areas and edema may be distinguished ideally by using the histograms of the signal intensities, which differ within the tumor and vary strongly in most sequences. Using combinations of histograms from different sequences the tumor can be described in a much better way than by calculating solely the tumor volume.

It is important to state that, despite recent diagnostic and therapeutic achievements, patients with glioblastoma have a poor prognosis and oncologists and neuroradiologists have to face multiple diagnostic problems. Early diagnosis of progressive disease (PD) and its distinction from different neuroradiologic phenomena, particularly radiation necrosis (RN), pseudoprogression (PP) and pseudoresponse (PR) is crucial since these entities request different treatment approaches [31]. To this end, several imaging techniques recently have been evaluated with varying results (see 5.2.1 Different Imaging Modalities) [10].

Texture analysis and feature extraction from images has been a long standing topic of radiologic research [58], reviving in some kind of a "texture renaissance" during the last decade [73]. Obviously this trend is related to the steep increase in digital technology and storage capability, computerized image management and the established acceptance of computer-aided diagnosis, engaging automatic or semiautomatic image analysis tools [140]. Stated by Kassner and Thornhill, Lerski et al. were the first to introduce MR image texture analysis for the characterization of intracranial tissues, the identification of brain tumors and the differentiation between them $[73,89]$. What Tourassi calls a "texture signature" refers to the idea of a reproducible and robust method of extracting pathologic findings from radiologic images, while being refractory to possible bias caused by individual attitude, beliefs, preconceptions, expectations or fatigue [140]. Especially in the evaluation of malignant brain tumors texture analysis seems to be a reasonable 
approach due to the limitations of image interpretation. With the basic mathematical method which we have engaged in our study we tried to obtain such a "texture signature", which may provide a worthwhile way to improve image analysis and to reveal new tumor-specific information that helps to distinguish between PD and the afore mentioned imaging phenomena.

Our results, gained by the use of the DoctorEye software, indicate that the proposed innovative method of texture analysis can be a complementary diagnostic tool to differentiate various intracranial lesions from each other as well as to assess response to treatment or progression in malignant glioma. At the time we initiated our study to the best of our knowledge no information was available that histograms of signal intensities from MR images were used for the characterization of glioblastoma. This is probably caused by the lack of standardization in MRI technologies, which hampers the comparability of the results.

Evaluating our results, the first thing we noticed was that at the time of diagnosis the histogram of all glioblastoma showed a bimodal distribution of signal intensities in T1 Gd-MRI sequence, with one peak each representing active tumor and necrosis. Figure 15 exemplarily illustrates our results from patient G-13 at the time of diagnosis. It is obvious that especially in the T1 Gd-MRI sequence the major part of the active tumor- and necrosis-area occupy different areas on the SI scale as well as their peaks do. As listed in Table III, the corresponding statistical values differ strongly from each other as well. The SI-peak of the active tumor is 93 on the SI scale and the mean SI 102,6, whereas for necrosis it is 63 and 63,8, respectively. These regularities of the SI distribution can also be found in the average histograms. Above all in the T1 Gd-MRI, but also in the other sequences, a clear distinction of the SI-peak and mean SI between active tumor and necrosis can be detected. Depending on the examined MR sequence, also edema can be discriminated easily either from active tumor or necrosis.

Such similarities of the shape and the statistical values are seen during the course of disease in individual patients as well. This is valid for the images after surgery, 
after radio- and chemotherapy and during the subsequent follow-up.

Nevertheless, we are faced with the problem of insufficient standardized procedures in the acquisition of MR images. To overcome this issue we normalized the mean SI of the vital tumor area and of the necrotic tumor area by relating them to the mean $\mathrm{SI}$ of the corresponding CSF, resulting in a clear difference between tumor and necrosis at all analyzed time points in T1, T1 GdMRI and T2 Flair (Table XVI). According to our observations, it seems that the more the standardized mean value of SI for the "whole lesion" in T1 with contrast enhancement is increasing during follow-up in a single patient, the more likely the patient suffers from disease progression or tumor recurrence. If this value is going down, the more likely a tumor response or a stable disease can be diagnosed. Typically after surgery we find an increase of the standardized SI and after radiation- and chemotherapy a decrease. Patient G-17 illustrates the SI development as an example of a patient suffering from tumor recurrence. While the SI after surgery is increasing, it decreases after radiation- and chemotherapy, as explained. Nevertheless, during follow-up the tumor is recurring, revealed by the increase of volume, already shown in Figure 28. Simultaneously to this volume changes, also the values for the SI peak, mean SI and standardized SI are increasing, as demonstrated in Figures 32-34. In contrast patient G-7 illustrates the course of disease from a patient with stable disease. Figure $\mathbf{3 0}$ depicts the volume development of this patient during follow-up, indicating a state of stable disease by the absence of any volume increase. Consistently also the SI values of patient G-7 (SI peak, mean SI and standardized SI) do not show any augmentation during follow up, as depicted in Figures 35-37.

This statistical distinctiveness is an ideal condition to develop an automatic segmentation algorithm, which would be the ideal advancement to increase the practicality of our approach. Therefore, we have involved Prof. Joachim Weickert from the Faculty of Mathematics and Computer Science at the Saarland University into our study. Prof. Weickert is an expert in the field of image processing and the head of the Mathematical Image Analysis Group (MIA) at the Saarland University. 
$\mathrm{He}$ and his team are working on a more sophisticated, automatic segmentation method to improve the accuracy and reproducibility of the results of our measurements.

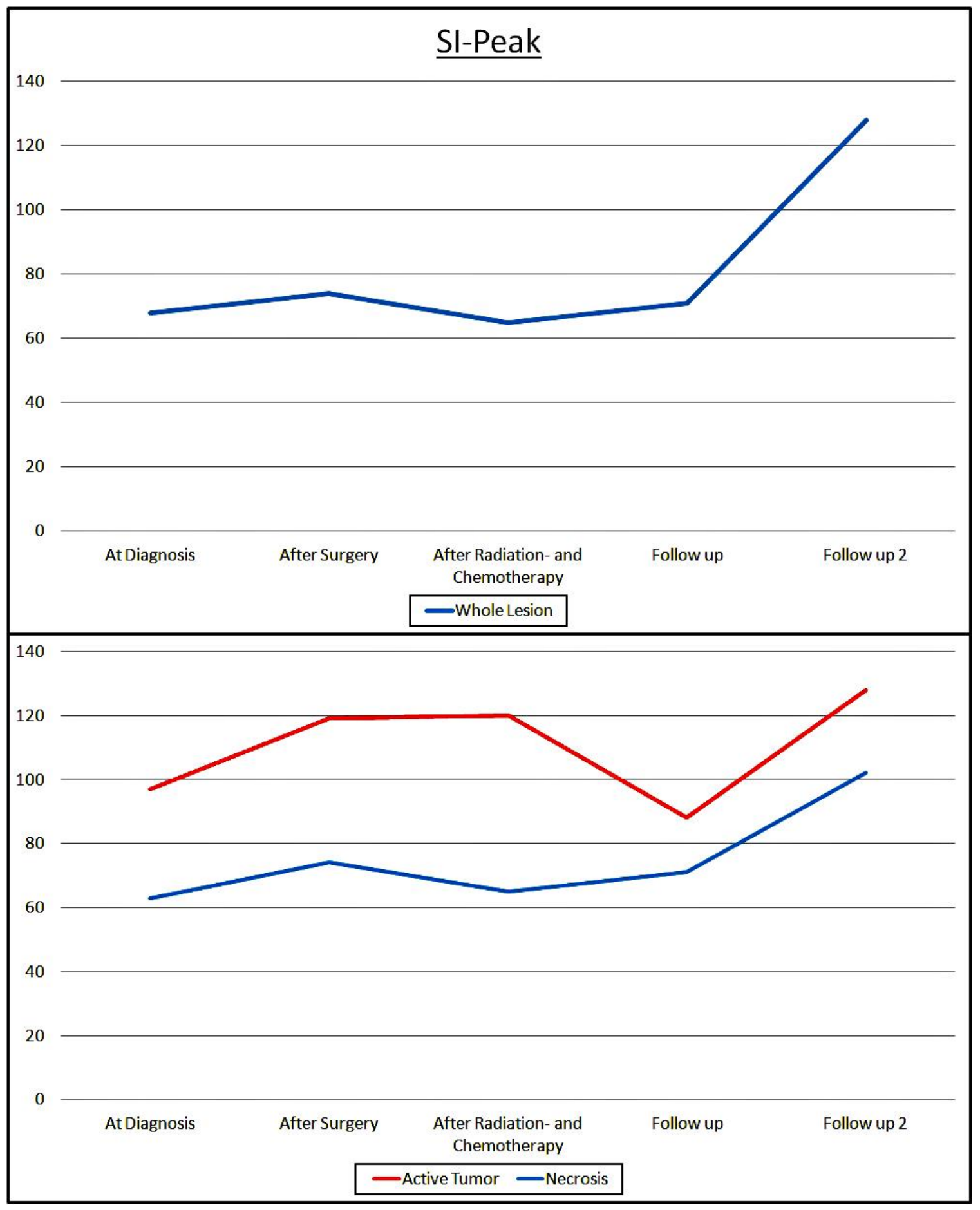

Figure 32: Development of SI-Peak during Follow-up in a Patient that suffers from Tumor Recurrence (G-17) 


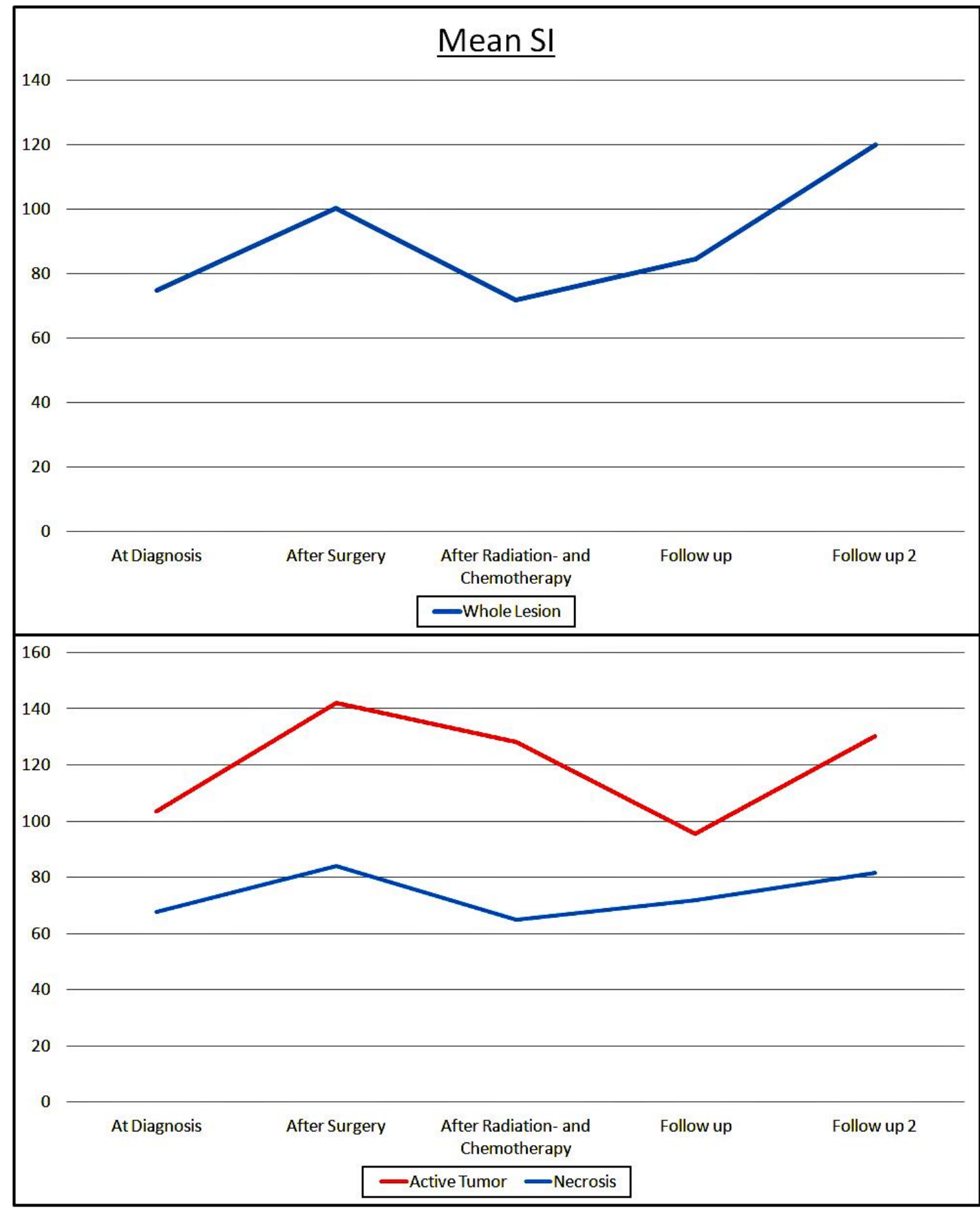

Figure 33: Development of Mean-SI during Follow-up in a Patient that suffers from Tumor Recurrence (G-17) 


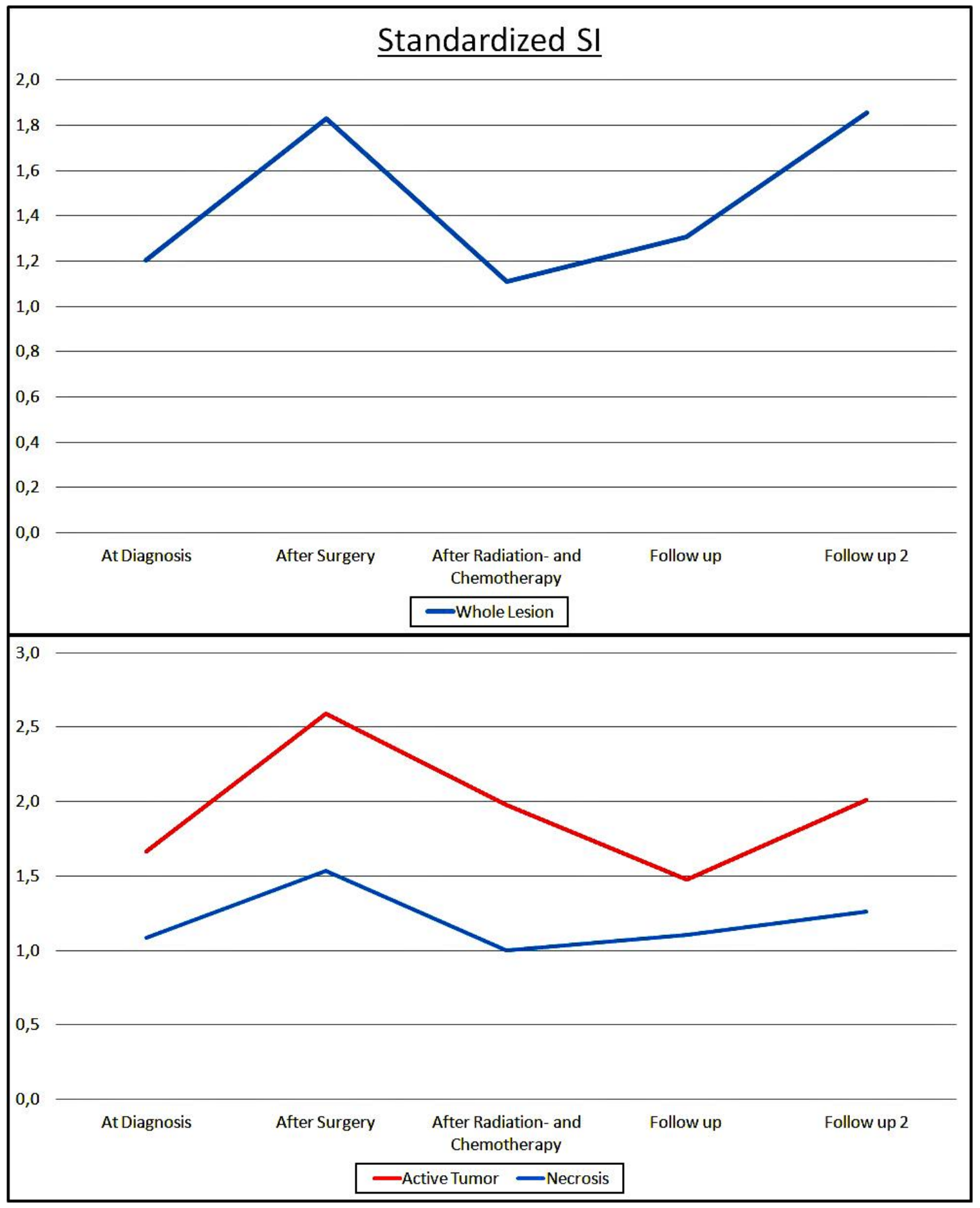

Figure 34: Development of Standardized-SI during Follow-up in a Patient that suffers from Tumor Recurrence (G-17) 


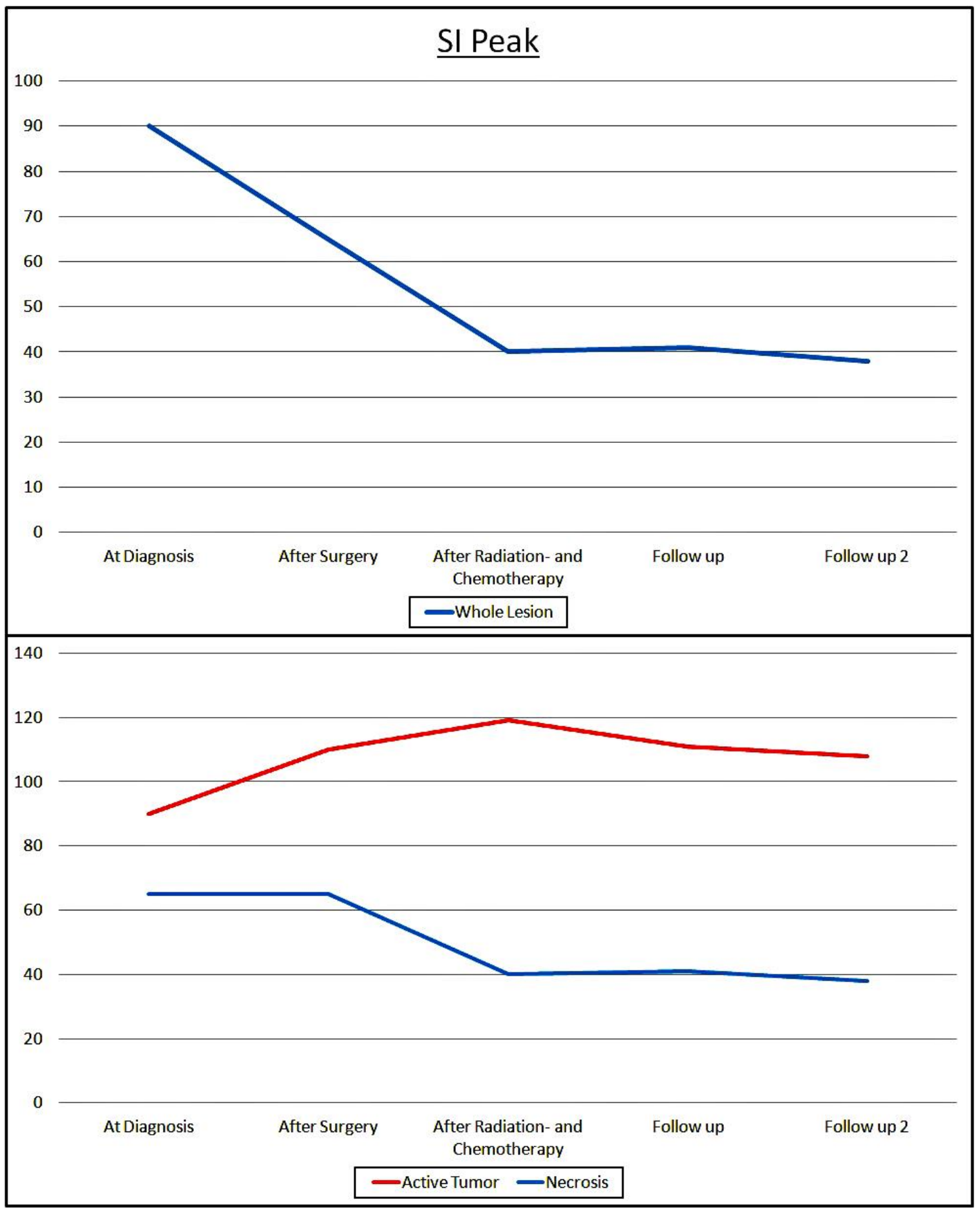

Figure 35: Development of SI-Peak during Follow-up in a Patient with stable Disease (G-7) 


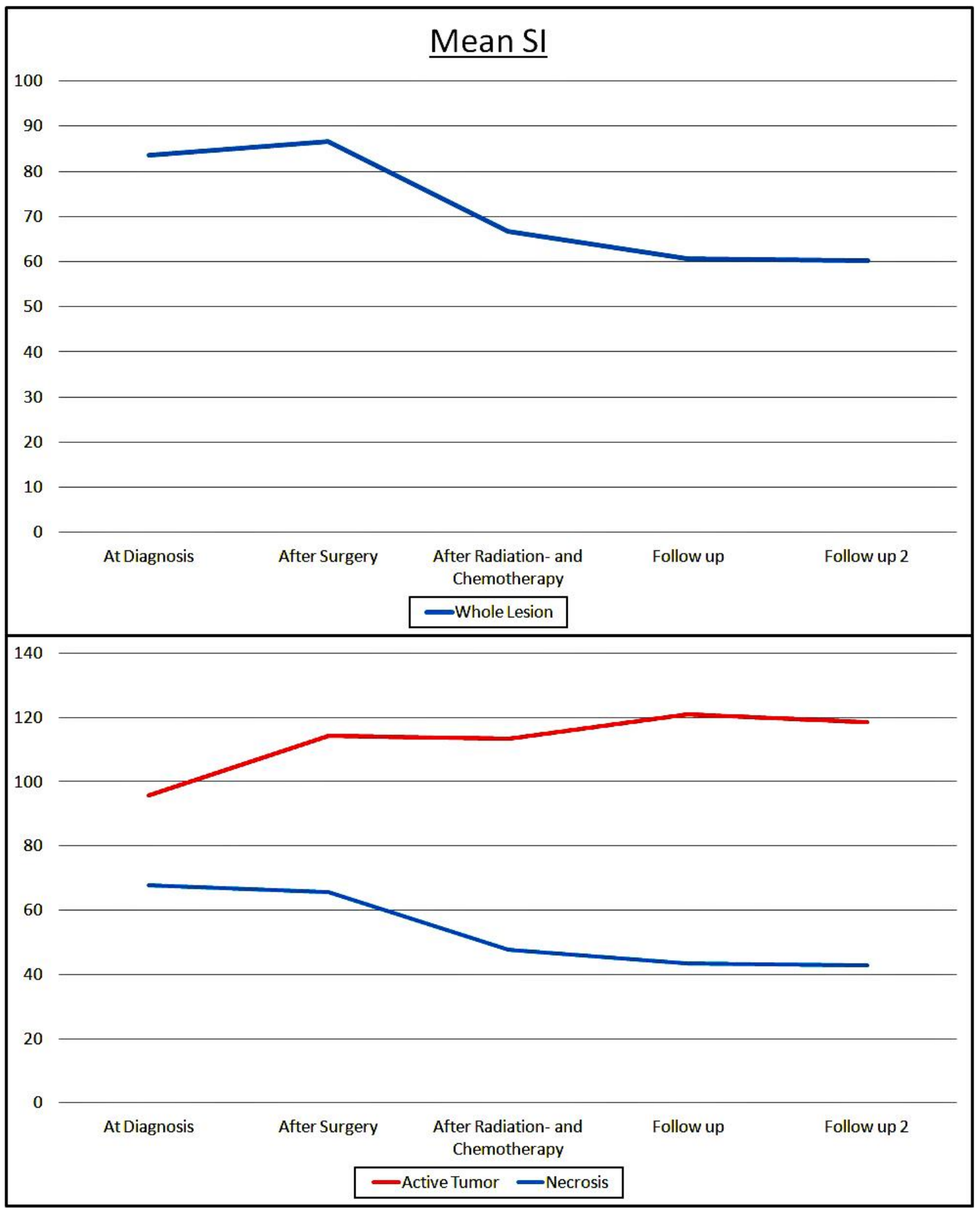

Figure 36: Development of Mean-SI during Follow-up in a Patient with stable Disease (G-7) 


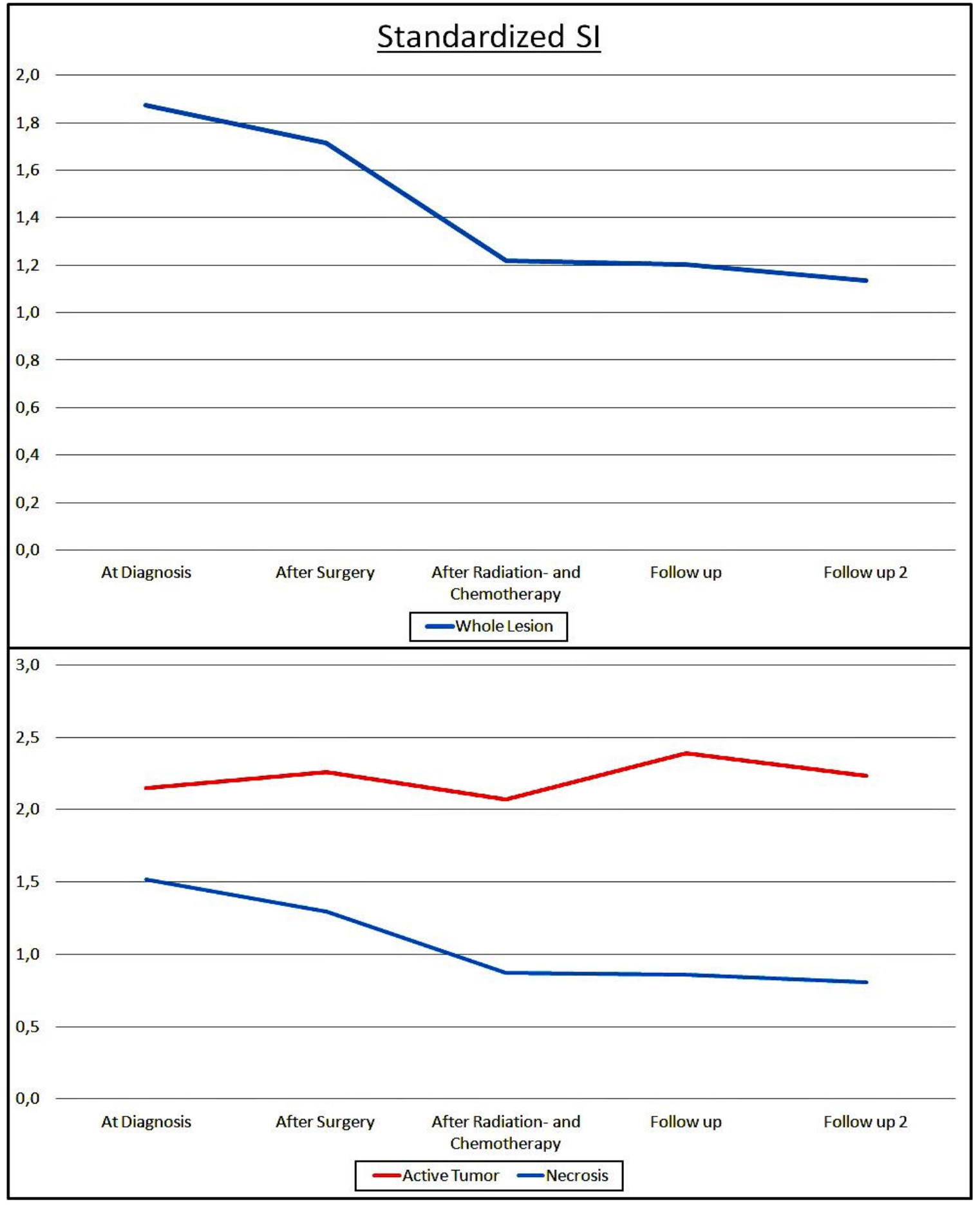

Figure 37: Development of Standardized SI during Follow-up in a Patient with stable Disease (G-7) 
Currently studies focusing also on the topic of texture analysis in GBM images have been published, e.g. by Drabycz et al., Pope et al. and Najafi et al.. In 2010 Drabycz et al. tried to noninvasively predict the MGMT methylation promotor status by texture analysis of MRI scans [46]. They could show that the ring enhancement on MR images from glioblastoma patients significantly correlated with an unmethylated MGMT promoter status. Nevertheless, their application of a space-frequency texture analysis did not deliver any valuable results [46]. Recently Pope et al. presented their results after testing the predictive value of apparent diffusion coefficient (ADC) histogram analysis, with the aim to prognosticate the progression-free survival (PFS) and overall survival (OS) in bevacizumab-treated patients suffering from recurrent GBM. In this attempt the histograms were computed from areas of enhancing tumor on T1 weighted postcontrast images. By the combined interpretation of the mean ADC from the lower curve (ADC-L) and the mean lower curve proportion (LCP) a maximum specificity of $87 \%$ for the prediction of progression-free survival was achieved [113]. Another topical approach was published by Najafi et al., trying to predict the response to bevacizumab treatment in patients with GBM [103]. Aspiring to extract significant statistical features especially from T1Gd-MRI, but also for T1-, T2- and T2 Flair-weighted images, they applied signal decomposition and histogram analysis methods. Volume estimation was accomplished for areas of gadoloniumenhancement, edema and necrosis. By comparing SI of T1-weigehted images and corresponding T1-postcontrast images they designed an elegant and innovative method for the normalization of the images and their grey levels. By analyzing the standard deviation of the grey matter histograms, it proved to provide a potentially significant tool to predict the response of GBM to bevacizumab treatment before it was initiated [103].

In future research the use of our technique especially for the discrimination of the radiologic phenomena described below has to be validated. It is our position that the extraction of information from routinely performed investigations has to be optimized and exhausted before creating the need for additional investigations, potentially at the expense of the patient's physical- and the hospital's 
economical-well-being. Hereinafter current neuroradiologic approaches towards these issues will be outlined. Furthermore, the mentioned phenomena are described in more detail.

\subsubsection{Different Imaging Modalities}

Today neuroradiologic imaging is the major diagnostic modality for the evaluation of brain tumors. The T1 Gd-MRI sequence is usually sufficient to suggest a brain tumor, while further sequences may already provide detailed information that indicates the specific tumor type. Currently there are several additional imaging modalities available, like magnetic resonance spectroscopy (MRS), diffusion weighted MRI (DWI), perfusion weighted imaging, positron emission tomography (PET), and single photon emission CT (SPECT). Nevertheless, to specify the diagnosis and to optimize the subsequent treatment strategy the acquisition of an adequate tissue sample by invasive procedures is essential. This will be acquired either at the time of the surgical resection of the tumor or by stereotactic biopsy, performed separately.

With the innovative mathematical method of analyzing routine MRI scans proposed by us we sought to reveal new tumor-specific information in order to facilitate the description of the tumor's development and possibly to differentiate between response to treatment and progressive disease. Furthermore, our approach could support the better identification of the different challenging radiologic phenomena like PP, PR and $\mathrm{RN}$, described below. To this end our method is easily feasible in daily clinical care and, since it can provide results without additional imaging studies, it proves to be more cost-effective. Nevertheless it has to be validated to prove its effectiveness and justification to be a diagnostic tool on the basis of conventional MRI, which is increasingly seen critical.

Concerning this matter Payer argues that conventional MRI is not sufficient to display the structural complexity, physiology and metabolism of GBMs. MRI may miss transformation and infiltration of healthy tissue [107]. Moreover, there is a risk 
to misinterpret changes of contrast enhancement as progression or therapy response, even if $\mathrm{PP}, \mathrm{RN}$ or $\mathrm{PR}$ represent the true cause of variation, or vice versa [107]. These limitations demand the introduction of more versatile procedures, imaging acquisition techniques on the one hand, which will be discussed in the following and image analysis techniques on the other hand, such as the one presented by us.

Three MR-based techniques have been established lately, to achieve more precise assessment of tumor development, DWI, perfusion-weighted imaging and MRS. DWI represents a dynamic method to display areas of water diffusion, using the apparent diffusion coefficient (ADC), thereby revealing lower diffusion values especially in areas of ischemia and areas of high cellularity [41]. Hein et al. demonstrated in 2004 that DWI could serve as a helpful tool in the differentiation between tumor progression and treatment-induced imaging changes, as recurrent tumor typically has significantly lower ADC values than RN [64]. Another advantage comes with the fact that DWI in many institutions is part of standard neuroimaging and therefore does not generate additional costs. In addition, the processing time can be kept short with the use of fast imaging sequences (echo planer imaging) and an integrated ADC calculating software in the workstation [64]. As Payer states, the introduction of MR-diffusion analysis in the clinical routine could enable to better characterize edema, necrosis and active tumor [107]. Perfusion weighted imaging allows the illustration of intracranial hemodynamics by assessing regional cerebral blood flow and blood volume, typically revealing an elevated microcirculation in high grade brain tumors [41]. In a small study $\mathrm{Hu}$ et al. indicated in 2009 that the relative cerebral blood volume ( $\mathrm{rCBV}$ ) can differentiate between recurrence and posttreatment radiation effects with a high degree of accuracy. According to their observation, recurrent tumor typically exhibits an elevated rCBV, whereas treatment induced contrast enhancement shows a reduced rCBV [68]. These findings are supported by a study of Baraja et al. that demonstrated with 57 patients suffering from GBM that rCBV was significantly higher in the group of recurrent tumor than within the radiation necrosis group [16]. Nevertheless, a considerable overlap for this 
parameter was noted between both groups, possibly due to the tumors heterogeneity. Therefore the notion seems reasonable that relative peak height $(\mathrm{rPH})$, which proved to be more reliable, could also be helpful in the differentiation between recurrent GBM and radiation necrosis, thereby complementing the noninvasive investigations [16]. In 2010 Mangla et al. suggested that the survival of patients with glioblastoma, after receiving combined radiation- and TMZ-therapy, shows a negative correlation with the rCBV. The increase of posttreatment rCBV seemed to be a strong predictor of poor survival and vice versa, whereas the Gd-MRI based McDonald-criteria did not appear to be useful in such a setting [97]. Innovative approaches of analyzing perfusion values, as proposed by Tsien et al., might also facilitate the differentiation between progressive disease and pseudoprogression [141]. Nevertheless, these methods are not accurate enough to be the only decisive value for distinguishing tumor from PP or RN, as Clarke et al. concludes [41]. MRS on the other hand reveals biochemical information about the concentration of brain metabolites like choline (Cho), N-acetylaspartate (NAA), creatine and lactate, with special emphasis on the Cho/NAA ratio. As Clarke et al. reviewed, an elevated Cho/NAA ratio, caused by an elevation of the Cho peak and a decrease of the NAA peak, can indicate active tumor. A typical indicative of necrosis is the general flattening of all peaks, whereas the overlap of two peaks corresponds to lipids and lactate. Nevertheless the most commonly available type of MRS, single-voxel MRS, is restricted in its clinical use due to the large size of the voxels. Although the introduction of multivoxel MRS improved precision, the significant heterogeneity caused by the voxel's size is still a problem. Moreover, the applicability is limited by the fact that the Cho/NAA ratio lacks in precision, as it may also be seen in the setting of significant inflammation or infection and therefore is not specific for progressive disease [41]. Nevertheless, in their review from 2012 Caroline and Rosenthal proposed that decreased Cho combined with a lipid or lactate peak indicates RN [31, 112]. In addition, recent literature provides studies that illustrate that sensitivity could be improved, if MRS was performed months after RT (89-100\%), while the results were more specific if it was performed in weeks after treatment (100\%) $[104,112,168]$. 
Payer reports that nuclear investigation methods through PET-based imaging can help to display the metabolism of glucose and amino acids in malignant gliomas by the use of radiotracers. There has been extensive research on the value of PET as a tool to distinguish between $P D$ and PP. Currently radiotracers like fluorodeoxyglucose $\left(\left[{ }^{18} \mathrm{~F}\right] \mathrm{FDG}\right)$, representing the glucose metabolism, as well as the amino acids methionine $\left(\left[{ }^{11} \mathrm{C}\right] \mathrm{MET}\right)$ and fluorothymidine $\left(\left[{ }^{18} \mathrm{~F}\right] \mathrm{FLT}\right)$ and the amino acid analog fluorethyltyrosin $\left(\left[{ }^{18} \mathrm{~F}\right] \mathrm{FET}\right)$, representing cellular and vascular proliferation, are widely available tracers [54, 107, 153]. Caroline and Rosenthal state that tissue hypermetabolism, visualized by an increased radiotracer uptake, is associated with tumor recurrence [31]. This hypothesis is supported by the study from Chao et al. published in 2001, where $\left[{ }^{18} \mathrm{~F}\right] \mathrm{FDG}$ was $75 \%$ sensitive and $81 \%$ specific in distinguishing recurrent brain tumor from RN [36]. However, la Fougere et al. argue that the sensitivity of $\left[{ }^{18} \mathrm{~F}\right] \mathrm{FDG}$, the most frequently used radiotracer, is significantly limited since many areas, such as the cerebral cortex, the basal ganglia, and the thalamus in the brain exhibit high physiologic glucose consumption, causing difficulties in the differentiation between tumor and normal brain. The same applies to the method's specificity due to the accumulation of $\left[{ }^{18} \mathrm{~F}\right] \mathrm{FDG}$ in macrophages and inflamed tissue, hampering the delineation of active tumor additionally [83]. Inspired by the knowledge of the importance of amino acid metabolization for the proliferation of glioma, radiolabeled amino acids were introduced as PET tracers in $1982[69,72,153]$. Due to the limitations of $\left[{ }^{18} \mathrm{~F}\right] \mathrm{FDG}$ as a PET tracer considerable research projects have engaged radiolabled amino acids, aiming to find tracers with both improved sensitivity and specificity. Already in 1996 Wurker et al. presented their results on the methionin uptake in patients with low-grade gliomas treated with brachytherapy, which suggested that amino acid tracers might be superior in monitoring therapeutic effects [162]. Regardless of the phase of the cell cycle it was shown experimentally that tumor cells are capable of up regulating the expression of amino acid transporters in their cell membrane in order to increase the transmembrane amino acid influx, particularly if growth conditions deteriorate [122]. Different studies proved that the distribution of $\left[{ }^{11} \mathrm{C}\right] \mathrm{MET}$ and $\left[{ }^{18} \mathrm{~F}\right] \mathrm{FET}$ in brain tumors is comparable [85], whereas the clinical availability of $\left[{ }^{18} \mathrm{~F}\right] \mathrm{FET}$ is superior due to its longer physical half life [153]. La 
Fougere et al. concluded that both markers, $\left[{ }^{11} \mathrm{C}\right] \mathrm{MET}$ and $\left[{ }^{18} \mathrm{~F}\right] \mathrm{FET}$ [83], hold high diagnostic power. For $\left[{ }^{11} \mathrm{C}\right.$ MET a sensitivity between 75 and $90 \%$ and a specificity between 72 and $92 \%$ for the detection of malignant transformation and its distinction to RN was demonstrated in different studies [137, 144, 146]. For $\left[{ }^{18} \mathrm{~F}\right] \mathrm{FET}$ a positive predictive value of $84 \%$, a sensitivity of $82 \%$ and a specificity $100 \%$ for the differentiation between tumor recurrence and reactive changes was reported $[100,114]$. To improve the accuracy of $\left[{ }^{18} \mathrm{~F}\right] \mathrm{FET}$ PET scans in the discrimination of tumor recurrence from posttherapeutic changes, Rachinger et al. analyzed the maximal standardized uptake value $\left(S U V_{\max }\right)$ for the slice with the highest FET uptake in the area with suspected tumor. A sensitivity of $100 \%$ and a specificity of $93 \%$ could be achieved for $\left[{ }^{18} \mathrm{~F}\right] \mathrm{FET}$ PET, which was significantly higher compared to the results of conventional MRI with $94 \%$ and $50 \%$, respectively [115]. Nevertheless, the damage of the blood-brain barrier could possibly be a source of misinterpretation, as the amino acid uptake might be governed by alteration of the blood brain barrier, causing an increased passive influx [119]. La Fougere conclude that although amino acid PET is not precise enough to replace histological verification yet, it provides additional information that might be helpful in the detection of glioma recurrence as well as to monitor new treatment modalities like antiangiogenic therapies [83].

CT today has widely been replaced by MRI as the imaging modality of choice in the initial assessment of intracranial lesions. Moreover, in recent years SPECT, using various isotopes to detect abnormalities in the blood brain barrier, shows promising results in the diagnosis of different types of intracranial lesions, the determination of residual or recurrent tumor and the assessment of response to treatment [135]. Currently utilized tracers are ${ }^{201}$ Thallium $\left({ }^{201} \mathrm{TI}\right)$, ${ }^{99 \mathrm{~m}}$ technetium $\left({ }^{99 \mathrm{~m}} \mathrm{Tc}\right.$ )-glucoheptonic-acid, $\quad{ }^{123}$ iodine $\left({ }^{123} \mathrm{l}\right)$-alpha-methyl-tyrosine and ${ }^{99 \mathrm{~m}}$ Tchexakis-2-methoxyisobutylisonitrile $\left({ }^{99 \mathrm{~m}} \mathrm{Tc}-\mathrm{MIBI}\right)$. These radionuclides possess longer half-lives than most PET tracers (e.g. $13,2 \mathrm{~h}$ for ${ }^{123} \mathrm{I}$ and $6 \mathrm{~h}$ for ${ }^{99 \mathrm{~m}} \mathrm{Tc}$, compared to $110 \mathrm{~min}$ for ${ }^{18} \mathrm{~F}$ and $20 \mathrm{~min}$ for ${ }^{11} \mathrm{C}$ ), which offers advantages like a facilitated chemical synthesis [167]. Generally a high tracer uptake in SPECT indicates PD, whereas a negative SPECT result suggests the absence of 
active tumor growth [31]. As Tie et al. state, in their study ${ }^{201} \mathrm{TI}$-SPECT provided a superior diagnostic accuracy to conventional $\mathrm{MRI}$ and could also serve as a potential prognostic tool, as low tracer uptake was reflected in a significantly longer post imaging survival time [139]. In the last decade many studies have been focusing on this topic. While ${ }^{201} \mathrm{TI}$ offers a $100 \%$ specificity and sensitivity in the range of $83-100 \%$ in distinguishing $\mathrm{RN}$ from $\mathrm{PD}[30,56,139],{ }^{99 \mathrm{~m}} \mathrm{Tc}$ and ${ }^{123}$ I showed comparable results trying to identify tumor recurrence with an accuracy of 93 and $96 \%$, respectively $[14,112] .{ }^{99 \mathrm{~m}} \mathrm{Tc}-\mathrm{MIBI}$ also has been investigated in various studies to detect recurrence with acceptable results; presenting a sensitivity of 93 and $89 \%$ and a specificity that was little lower with 83 and $83 \%$ $[87,164]$. As Caroline and Rosenthal point out, the major advantage of Thallium is its exclusive uptake in pathologic tissue and not in healthy tissue, as it is found with other tracers. Nevertheless, certain limitations, like spatial resolution and low energy photon emission, are restricting the administration dose [15, 31]. Technetium on the other hand is not restricted by these limitations, but shows deficits in image interpretation, as the choroid plexus and the temporalis and extraocular muscles demonstrate an intense physiologic tracer uptake [15].

Two studies cited by Caroline and Rosenthal suggest that the combination of imaging methods might offer additional benefits detecting tumor recurrence. Gomez et al. could demonstrate in 2008 that combined ${ }^{201} \mathrm{TI}$-SPECT and MRI were more sensitive (97\%) than the combination of ${ }^{18} \mathrm{~F}$-FDG PET and MRI (83\%) [56]. Van Laere et al. proposed in 2005 that the use of $\left[{ }^{18} \mathrm{~F}\right] \mathrm{FET}$ PET combined with $\left[{ }^{11} \mathrm{C}\right] \mathrm{MET}$ presented higher accuracy $(83 \%)$ than using each one of them solely (80 and 73\%) [146].

As these results show, there is still potential for improvement and the need for innovative ideas, as proposed by us, to assess the development of this disease more precisely, without burdening the patients with additional investigations and the hospitals with additional costs. 


\subsubsection{Imaging Phenomena: Pseudoprogression, Radiation Necrosis and Pseudoresponse}

Different treatment induced radiologic phenomena have hampered the work of radiologists and oncologists in interpreting images of intracranial lesions, especially the so called pseudoprogression (PP), radiation necrosis (RN) and pseudoresponse (PR).

The term "Pseudoprogression" was originally inaugurated by Hoffman et al. in 1979 [66] and characterizes treatment-induced imaging changes, occurring particularly in the period immediately following completion of RT [19]. It is believed that PP is caused by cytotoxic effects of chemotherapy and radiation, resulting in a subacute inflammatory response with abnormal vessel permeability and local edema. Up to $20 \%$ of the patients develop this phenomenon after treatment with TMZ chemoradiotherapy. It is believed that in the case of PP the patients often remain asymptomatic and the image changes decrease in size or normalize without additional interventions [26]. De Witt et al. revisited this phenomenon in the year 2004 in patients with newly diagnosed GBM treated with RT and with or without carmustine [43]. They showed that from all patients presenting with disease progression immediately after RT ( $28 \%$ to $51 \%$ ), more than half ( $28 \%$ to $33 \% ; 9 \%$ to $14 \%$ of the total) subsequently either improved or demonstrated stable brain imaging.

With the aim to identify certain characteristics, Chamberlain et al. analyzed this phenomenon with the principles of pathology [33]: Within all patients 50\% showed progressive disease of which $50 \%$ were treated surgically (25\% of the total). Remarkably, half of the re-operated patients ( $14 \%$ of the total) displayed treatment injury without identifiable glioblastoma tissue. Hence, PP was characterized as to appear mainly (approximately 58\%) within the first 3 months after completing RT plus TMZ. Nevertheless, this cannot be interpreted as an average course of disease, as only one third of all patients have been reinvestigated within the first 3 month after the completion of RT plus TMZ. Taal et al. investigated the incidence of early PP and its clinical features, based on the MacDonald Criteria, by reviewing 
a cohort of patients with newly diagnosed malignant gliomas, who were treated with RT plus concomitant and adjuvant TMZ [136]. In this retrospective study 36 of 85 patients (42\% of the total) exhibited early disease progression in the first followup scan 4 weeks after the end of RT. PP was diagnosed in 18 of these 36 patients (50\%). Brandes et al. analyzed the phenomenon with regard to the tumor's MGMT promotor methylation status [25]. In 103 reviewed patients 36 (35\%) were found with MGMT promotor methylation. Of the 50 patients who showed early progression at the first MRI scan subsequently 32 were classified with PP. Within 23 patients with methylated MGMT promotor and early disease progression 21 (91\%) were recorded as PP. The detection of PP and of MGMT status both significantly influenced survival.

Commenting on Brandes et al. study from 2008 and referring to the mentioned studies, M.C. Chamberlain concludes that PP is characterized by signs of early disease recurrence seen on Gd-MR scans immediately post-RT plus TMZ with improvement or stabilization after 2 months [32].

Radiation Necrosis was first reported in 1930 in a case of basal cell epithelioma [51] and has been initially described as a side effect following radiation therapy of extracranial malignancies [55]. It can occur between a few months and many years post-irradiation. Most cases present within a six-month to two-year period, the time during which tumor recurrence is also most frequent [10]. It most often can be found around blood vessels within the white matter. Typical histopathologic findings in the vessel walls are fibrinoid necrosis followed by hyalinization with consecutive luminal narrowing caused by wall thickening. Surrounding perivascular coagulative necrosis have the potential to coalesce, forming large areas of parenchymal necrosis with subsequent deposition of mineral salts (ie, dystrophic calcification). Additionally, abnormal telangiectasias, as well as focal and diffuse demyelination of the white matter, can be observed [81]. For a standard therapy course with a cumulative dose of 60 Gy and 1,8-2 Gy per single dose an incidence of $3-5 \%$ has been shown [107]. If higher dosages or additional treatments as brachytherapy, experimental chemotherapy protocols or stereotactic 
RT following standard RT are applied, the chance to develop RN can rise up to $40 \%$ [107, 121]. Moreover, Chamberlain et al. in 2007 presented a study of 51 patients who received the standard of care for patients with newly diagnosed GBM (TMZ given concurrently with RT followed by 6 monthly cycles of TMZ), which indicates that daily administration of TMZ may represent a strong radio-sensitizing regimen. They reported that from 15 patients who underwent re-operation within 6 months of completion of RT for suspected progressive GBM almost 50\% (7 patients) showed histologically confirmed necrosis without evidence of active tumor (14\% of the whole study group) [33]. These results support the assumption that the RN and PP cannot be separated clearly and that PP might just be a mild, self-limited type of therapy-induced necrosis. Usually the radiation-related injury is placed around the initial tumor bed, but can also occur in other areas included in the radiation field [10]. As the clinical symptoms are very inconsistent they easily can mimic tumor recurrence. Most commonly observed are clinical signs as headache, seizures, personality changes and neurologic deficits [110].

Another radiologic phenomenon caused by the use of antiangiogenetic drugs was named by the term Pseudoresponse or Pseudoregression. Because hypervascularisation by the help of VEGF plays a critical role in the genesis of malignant gliomas, recent approaches include antiangiogenetic agents, especially those targeting VEGF, such as bevacizumab, and the VEGF receptor, such as cediranib. The use of such agents like the monoclonal antibody bevacizumab, which binds VEGF and by that is able to normalize the tumor vasculature, can cause marked decrease in contrast enhancement as early as 1 to 2 days after initiation of therapy and commonly result in high radiologic response rates of $25 \%$ to $60 \%$, a rate that was never documented before $[21,52,79,148]$. However, the conclusion of a tumor regression appears to be doubtful. Even if the imaging implies a tumor regression the effect on overall survival is limited, which suggests that these changes may not correlate with actual tumor regression. The clinical significance of this phenomenon was shown in 2010 by Wick et al., who highlighted the discrepancy between high response rate (measured by MacDonald Criteria) and the comparatively modest survival benefit [160]. The apparent 
response mostly is due to normalization of the tumors blood-brain barrier, rather than to a real anti-tumorous effect. It is common that normalization like that might cause decrease of perifocal edema, which consequently leads to neurological improvements [20, 24].

This effect proves another aspect where the MacDonald Criteria fail to be adequate, namely in not taking nonenhancing tumors into account [159]. Norden et al. even imply that in this context progression-free-survival might be an inappropriate endpoint for phase II trials [105].

As described in 5.1, using the DoctorEye software is an elegant way of analyzing changes in the texture of routine MRI scans. There is a chance that by testing our method on proven cases of PP, RN and PR a new diagnostic procedure could be developed to address these radiologic issues, as implied in chapters 4.2.3 and 4.2.5. This procedure would be based on the use of DoctorEye, the incorporation of all four MR sequences into the diagnosis process and the combined interpretation of SI-histograms and specific volume-developments, which is described in the following chapter.

\subsubsection{Volumetric Measurements}

The approximation of tumor size is often used as an end point in clinical trials of oncology therapy. Traditionally it has been assessed by measuring the crosssectional area, as suggested by the McDonald-criteria, which is calculated as the product of the lesion's longest diameter and its longest perpendicular diameter [96]. Especially in our study these criteria appeared inappropriate, as they bear a high risk of error due to the typically irregular shaped GBM lesions and the regularly included necrotic areas. Therefore, we decided to determine tumorvolume measurement as described in chapter 3.3.5 Statistical Analysis. Since we did not only investigate our segmentations of the whole tumor, but also of the subunits active tumor, necrotic area and edema, we were able to calculate the specific volume for each of these single qualities and compare them with each other and the whole lesion over the course of time. This allowed us to evaluate the 
volume-development from different patients, different tumor qualities and sequences at the time of diagnosis and during follow-up. Especially in the evaluation of the follow-up data this gain of presumably objective, complementary information might be of great value. The precise knowledge of the volumetric development of active tumor, necrosis and edema could be a helpful tool in the explanation of individual symptoms caused by the lesion, in the assessment of response or reaction to different treatment strategies and in the estimation of prognosis and survival. In addition this approach could provide a new end point in clinical trials in oncology. Nevertheless, to prove the reliability and the usefulness as a prognostic marker, further validation has to be done.

In the study published by Ertl-Wagner et al. in 2009 the authors assessed the reliability of tumor volume estimation by two semi-automatic segmentation methods and a manual method, comparing the results of 16 independent readers. They could show that semi-automated software systems are more reliable than manual measurements [48]. Challenging the classical cross-sectional volume estimation on T1 postcontrast images, proposed by the McDonald criteria, Wang et al. investigated the value of software supported volume measurement in high resolution 3D MRI [149]. Even though volumetric measurement allows a more accurate estimation of irregular shaped tumors, the results of cross sectional area measurement were almost comparable and revealed a high correlation. Nevertheless, Wang et al. underline that cross sectional area measurement retains the already mentioned limitations and that volumetric measurements might be more accurate, as confirmed in several other studies [45, 65, 71, 129, 163], whereas the proposed method was much more time consuming. Even though tumor volume might not be a prognostic factor, as shown by lliadis et al., it still is of important oncologic value [71].

\subsubsection{Tumor Modeling}

On the threshold of a new era of personalized medicine, the use of in silico cancer modeling is gaining more and more importance [44]. Nevertheless, just a few groups investigate simulation models based on MR technologies for the prediction 
of tumor response in GBM [37, 150, 169]. Chen et al. could show in 2010 that such simulations are able to successfully predict the region of recurrence in glioblastoma. Precise data from imaging studies are of utmost importance to gain such results in silico oncology models. The better these data are the more accurate predictions can be achieved [130,131]. For the validation of the models the analysis of segmentations from tumor images at diagnosis and during followup is of utmost importance. A correlation between tumor texture and signal intensities in MRI expressed by histograms of signal intensities is a step forward in precisely differentiating between and calculating volumes of different tumor areas, e.g. necrotic and vital tumor. The value of such histogram data in in-silico oncology models and the onco-simulator is under investigation in different EU funded projects [6, 7]. The results of our study and our influence on the development of the DoctorEye software made a great contribution to the progress of these projects.

\subsection{Limitations and Perspectives}

To start with the limitations of our approach we had to realize, that the interindividual comparability of our results, i.e. from one patient to another or from one MRI study to another, was limited due to the still insufficient standardization of technical procedures in neuroimaging. For example, various diagnostic centers often use different MRI technology and settings. Moreover, even within the same center there often is a lack of standardization due to different preferences for operating procedures. In addition, the semiautomatic rendering we had to engage, proved to be very time consuming if high accuracy was required.

On the other hand, the new methodological approach we investigated has the potential to provide important new information, without the need to perform additional investigations of the patient. Besides offering more convenience for the patient, this also spares time and money. Furthermore, we could gain objective data as a basis for the modeling of tumor growth or therapeutic response. It also could provide a complementary tool for objective documentation and follow-up of a 
patient's clinical record. As GBM exhibits a typical bimodal distribution of signal intensities it can be questioned whether other brain tumor entities express different shapes of the histogram of signal intensities as well. If shown to be so, this method would support a better characterization of brain tumors by MRI.

Nevertheless, before this method can be transferred into routine clinical praxis, several prerequisites have to be provided, as mentioned earlier. First of all an improved standardization of the imaging procedures is of utmost importance as well as the extended validation of our results, for example by the help of PETscans. Finally, as human beings represent a very slow performing and potentially imprecise rendering device, an automatic segmentation tool definitely would improve the clinical applicability of the method. Therefore, with the help of our partners we are working on solutions for these issues. 


\section{References}

1. Amiravis. Available from: http://www.amiravis.com/.

2. Annotor. Available from:

http://www.montefiore.ulg.ac.be/services/stochastic/biomod/doku.php?id=so ftware.

3. Internet Analysis Tools Registry. Available from:

http://www.cma.mgh.harvard.edu/iatr/display.php?spec=all\#hbp.

4. Itk-SNAP. Available from: http://www.itksnap.org/.

5. NeuroLib - Library for Image Processing. Available from: http://www.ia.unc.edu/dev/download/index.htm.

6. p-medicine. 22.09.2012; Available from: http://p-medicine.eu.

7. TUMOR. Available from: http://tumor-project.eu.

8. AG, S. MAGNETOM Vision. 2002-2013; Available from: http://www.medical.siemens.com/webapp/wcs/stores/servlet/PSGenericDis play?storeld=10001\&langld=-1\&catalogld=-1\&pageld=77335.

9. Alberta-Provincial-CNS-Tumor-Team (2009) Clinical Practice Guideline Management of Glioblastoma Multiforme. Alberta Cancer Board.

10. Alexiou, G.A., S. Tsiouris, A.P. Kyritsis, S. Voulgaris, M.I. Argyropoulou, A.D. Fotopoulos (2009) Glioma recurrence versus radiation necrosis: accuracy of current imaging modalities. Journal of Neuro-Oncology. 95(1): 1-11.

11. Andersen, A.P. (1978) Postoperative irradiation of glioblastomas. Results in a randomized series. Acta Radiol Oncol Radiat Phys Biol. 17(6): 475-84.

12. Athanassiou, H., M. Synodinou, E. Maragoudakis, M. Paraskevaidis, C. Verigos, D. Misailidou, D. Antonadou, G. Saris, K. Beroukas, P. Karageorgis (2005) Randomized phase II study of temozolomide and radiotherapy compared with radiotherapy alone in newly diagnosed glioblastoma multiforme. J Clin Oncol. 23(10): 2372-7.

13. Atlas, S.W. (2009) Magnetic Resonance Imaging of the Brain and Spine. 4th ed: Lippincott Williams \& Wilkins.

14. Barai, S., G.P. Bandopadhayaya, P.K. Julka, K.K. Naik, A.K. Haloi, R. Kumar, A. Seith, A. Malhotra (2004) Role of ${ }^{99 m}$ Tc-glucoheptonic acid brain single photon emission computed tomography in differentiation of recurrent 
brain tumour and post-radiation gliosis. Australasian Radiology. 48(3): 296301.

15. Barai, S., Rajkamal, G.P. Bandopadhayaya, G.S. Pant, A.K. Haloi, A. Malhotra, H. Dhanpathi (2005) Thallium-201 versus Tc99m-glucoheptonate SPECT for evaluation of recurrent brain tumours: a within-subject comparison with pathological correlation. J Clin Neurosci. 12(1): 27-31.

16. Barajas, R.F., Jr., J.S. Chang, M.R. Segal, A.T. Parsa, M.W. McDermott, M.S. Berger, S. Cha (2009) Differentiation of recurrent glioblastoma multiforme from radiation necrosis after external beam radiation therapy with dynamic susceptibility-weighted contrast-enhanced perfusion MR imaging. Radiology. 253(2): 486-96.

17. Batchelor, T., W.T. Curry. Clinical manifestations and initial surgical approach to patients with malignant gliomas. 2011 Aug 31, 2011; Available from: www.uptodate.com.

18. Batchelor, T., D. Louis. Pathogenesis and biology of malignant gliomas. 2012 Feb 14, 2012; Available from: www.uptodate.com.

19. Batchelor, T., H.A. Shih, B.S. Carter. Management of recurrent malignant gliomas. 2012 Jul 31, 2012; Available from: www.uptodate.com.

20. Batchelor, T.T., D.G. Duda, E. di Tomaso, M. Ancukiewicz, S.R. Plotkin, E. Gerstner, A.F. Eichler, J. Drappatz, F.H. Hochberg, T. Benner, D.N. Louis, K.S. Cohen, H. Chea, A. Exarhopoulos, J.S. Loeffler, M.A. Moses, P. Ivy, A.G. Sorensen, P.Y. Wen, R.K. Jain (2010) Phase // study of cediranib, an oral pan-vascular endothelial growth factor receptor tyrosine kinase inhibitor, in patients with recurrent glioblastoma. Journal of Clinical Oncology (JCO). 28(17): 2817-23.

21. Batchelor, T.T., A.G. Sorensen, E. di Tomaso, W.T. Zhang, D.G. Duda, K.S. Cohen, K.R. Kozak, D.P. Cahill, P.J. Chen, M. Zhu, M. Ancukiewicz, M.M. Mrugala, S. Plotkin, J. Drappatz, D.N. Louis, P. Ivy, D.T. Scadden, T. Benner, J.S. Loeffler, P.Y. Wen, R.K. Jain (2007) AZD2171, a pan-VEGF receptor tyrosine kinase inhibitor, normalizes tumor vasculature and alleviates edema in glioblastoma patients. Cancer Cell. 11(1): 83-95.

22. Bauer, S., L.P. Nolte, M. Reyes. Fully automatic segmentation of brain tumor images using support vector machine classification in combination with hierarchical conditional random field regularization. in Medical Image Computing and Computer-Assisted Intervention - MICCAI 2011. 2011. Toronto, Canada.

23. Bendszus, M., M. Platten [Neuroradiological response criteria for malignant gliomas]. Nervenarzt. 81(8): 950-5. 
24. Bendszus, M., M. Platten (2010) [Neuroradiological response criteria for malignant gliomas]. Nervenarzt. 81(8): 950-5.

25. Brandes, A.A., E. Franceschi, A. Tosoni, V. Blatt, A. Pession, G. Tallini, R. Bertorelle, S. Bartolini, F. Calbucci, A. Andreoli, G. Frezza, M. Leonardi, F. Spagnolli, M. Ermani (2008) MGMT promoter methylation status can predict the incidence and outcome of pseudoprogression after concomitant radiochemotherapy in newly diagnosed glioblastoma patients. Journal of Clinical Oncology (JCO). 26(13): 2192-7.

26. Brandsma, D., L. Stalpers, W. Taal, P. Sminia, M.J. van den Bent (2008) Clinical features, mechanisms, and management of pseudoprogression in malignant gliomas. Lancet Oncology. 9(5): 453-61.

27. Buatti, J., T.C. Ryken, M.C. Smith, P. Sneed, J.H. Suh, M. Mehta, J.J. Olson (2008) Radiation therapy of pathologically confirmed newly diagnosed glioblastoma in adults. Journal of Neuro-Oncology. 89: 313-337.

28. Burger, P.C., E.R. Heinz, T. Shibata, P. Kleihues (1988) Topographic anatomy and CT correlations in the untreated glioblastoma multiforme. J Neurosurg. 68(5): 698-704.

29. Cairncross, J.G., J.H. Pexman, M.P. Rathbone, R.F. DelMaestro (1985) Postoperative contrast enhancement in patients with brain tumor. Ann Neurol. 17(6): 570-2.

30. Caresia, A.P., J. Castell-Conesa, M. Negre, A. Mestre, G. Cuberas, A. Manes, X. Maldonado (2006) Thallium-201SPECT assessment in the detection of recurrences of treated gliomas and ependymomas. Clinical and Translational Oncology. 8(10): 750-4.

31. Caroline, I., M.A. Rosenthal (2012) Imaging modalities in high-grade gliomas: pseudoprogression, recurrence, or necrosis? Journal of Clinical Neuroscience. 19(5): 633-7.

32. Chamberlain, M.C. (2008) Pseudoprogression in glioblastoma. J Clin Oncol. 26(26): 4359; author reply 4359-60.

33. Chamberlain, M.C., M.J. Glantz, L. Chalmers, A. Van Horn, A.E. Sloan (2007) Early necrosis following concurrent Temodar and radiotherapy in patients with glioblastoma. Journal of Neuro-Oncology. 82(1): 81-3.

34. Chang, S.M., I.F. Parney, W. Huang, F.A. Anderson, Jr., A.L. Asher, M. Bernstein, K.O. Lillehei, H. Brem, M.S. Berger, E.R. Laws (2005) Patterns of care for adults with newly diagnosed malignant glioma. JAMA. 293(5): 557-64.

35. Chang, S.M., I.F. Parney, M. McDermott, F.G. Barker, 2nd, M.H. Schmidt, W. Huang, E.R. Laws, Jr., K.O. Lillehei, M. Bernstein, H. Brem, A.E. Sloan, 
M. Berger (2003) Perioperative complications and neurological outcomes of first and second craniotomies among patients enrolled in the Glioma Outcome Project. J Neurosurg. 98(6): 1175-81.

36. Chao, S.T., J.H. Suh, S. Raja, S.Y. Lee, G. Barnett (2001) The sensitivity and specificity of FDG PET in distinguishing recurrent brain tumor from radionecrosis in patients treated with stereotactic radiosurgery. International Journal of Cancer. 96(3): 191-197.

37. Chen, L.L., S. Ulmer, T.S. Deisboeck (2010) An agent-based model identifies MRI regions of probable tumor invasion in a patient with glioblastoma. Physics in Medicine and Biology. 55(2): 329-338.

38. Chernoff, D., P. Stark. Principles of magnetic resonance imaging. 2012 05.04.12; Available from: http://www.uptodate.com.

39. Choubey, M., S. Agrawal (2012) A Fully Automatic Approach to Detect Brain Cancer Using Random Walk Algorithm. International Journal of Computer Technology and Applications. 03(01): 265-268.

40. Clark, M.C., L.O. Hall, D.B. Goldgof, R. Velthuizen, F.R. Murtagh, M.S. Silbiger (1998) Automatic tumor segmentation using knowledge-based techniques. IEEE Trans Med Imaging. 17(2): 187-201.

41. Clarke, J.L., S.M. Chang (2012) Neuroimaging: diagnosis and response assessment in glioblastoma. The Cancer Journal. 18(1): 26-31.

42. Corso, J.J., E. Sharon, S. Dube, S. El-Saden, U. Sinha, A. Yuille (2008) Efficient multilevel brain tumor segmentation with integrated bayesian model classification. IEEE Trans Med Imaging. 27(5): 629-40.

43. de Wit, M.C., H.G. de Bruin, W. Eijkenboom, P.A. Sillevis Smitt, M.J. van den Bent (2004) Immediate post-radiotherapy changes in malignant glioma can mimic tumor progression. Neurology. 63(3): 535-7.

44. Deisboeck, T.S., L. Zhang, J. Yoon, J. Costa (2009) In silico cancer modeling: is it ready for prime time? Nature Clinical Practice Oncology. 6(1): 34-42.

45. Dempsey, M.F., B.R. Condon, D.M. Hadley (2005) Measurement of tumor "size" in recurrent malignant glioma: 1D, 2D, or 3D? AJNR American Journal of Neuroradiology. 26(4): 770-776.

46. Drabycz, S., G. Roldan, P. de Robles, D. Adler, J.B. McIntyre, A.M. Magliocco, J.G. Cairncross, J.R. Mitchell (2010) An analysis of image texture, tumor location, and MGMT promoter methylation in glioblastoma using magnetic resonance imaging. Neuroimage. 49(2): 1398-1405.

47. Eisenhauer, E.A., P. Therasse, J. Bogaerts, L.H. Schwartz, D. Sargent, R. 
Ford, J. Dancey, S. Arbuck, S. Gwyther, M. Mooney, L. Rubinstein, L. Shankar, L. Dodd, R. Kaplan, D. Lacombe, J. Verweij (2009) New response evaluation criteria in solid tumours: revised RECIST guideline (version 1.1). Eur J Cancer. 45(2): 228-47.

48. Ertl-Wagner, B.B., J.D. Blume, D. Peck, J.K. Udupa, B. Herman, A. Levering, I.M. Schmalfuss (2009) Reliability of tumor volume estimation from MR images in patients with malignant glioma. Results from the American College of Radiology Imaging Network (ACRIN) 6662 Trial. European Radiology. 19(3): 599-609.

49. Farmaki, C., A spatially adaptive active contour approach for improving semi-automatic cancer image segmentation, in Department of Computer Science. 2009, University of Crete: Heraklion.

50. Farmaki, C., K. Marias, V. Sakkalis, N. Graf (2010) Spatially adaptive active contours: a semi-automatic tumor segmentation framework. International Journal of Computer Assisted Radiology and Surgery. 5(4): 369-384.

51. Fischer, A.W., Holfelder (1930) Lokales Amyloid im Gehirn. Deutsche Zeitschrift für Chirurgie. 227(1): 475-483.

52. Friedman, H.S., M.D. Prados, P.Y. Wen, T. Mikkelsen, D. Schiff, L.E. Abrey, W.K. Yung, N. Paleologos, M.K. Nicholas, R. Jensen, J. Vredenburgh, J. Huang, M. Zheng, T. Cloughesy (2009) Bevacizumab alone and in combination with irinotecan in recurrent glioblastoma. Journal of Clinical Oncology (JCO). 27(28): 4733-40.

53. Galanis, E., J.C. Buckner, M.J. Maurer, R. Sykora, R. Castillo, K.V. Ballman, B.J. Erickson (2006) Validation of neuroradiologic response assessment in gliomas: measurement by RECIST, two-dimensional, computer-assisted tumor area, and computer-assisted tumor volume methods. Neuro Oncol. 8(2): 156-65.

54. Gerstner, E.R., A.G. Sorensen, R.K. Jain, T.T. Batchelor (2008) Advances in neuroimaging techniques for the evaluation of tumor growth, vascular permeability, and angiogenesis in gliomas. Current Opinion in Neurology. 21(6): 728-735.

55. Glass, J.P., T.L. Hwang, M.E. Leavens, H.I. Libshitz (1984) Cerebral radiation necrosis following treatment of extracranial malignancies. Cancer. 54(9): 1966-72.

56. Gomez-Rio, M., A. Rodriguez-Fernandez, C. Ramos-Font, E. LopezRamirez, J.M. Llamas-Elvira (2008) Diagnostic accuracy of 201ThalliumSPECT and 18F-FDG-PET in the clinical assessment of glioma recurrence. European Journal of Nuclear Medicine and Molecular Imaging. 35(5): 966 75. 
57. Grosu, A.L., M. Bamberg (2011) Gliome. Der Onkologe. 17: 6-8.

58. Hall, E.L., R.P. Kruger, S.J. Dwyer, D.L. Hall, R.W. Mclaren, G.S. Lodwick (1971) A Survey of Preprocessing and Feature Extraction Techniques for Radiographic Images. IEEE Transactions on Computers. C-20(9): 10321044.

59. Halperin, E.C., J. Herndon, S.C. Schold, M. Brown, N. Vick, J.G. Cairncross, D.R. Macdonald, L. Gaspar, B. Fischer, E. Dropcho, S. Rosenfeld, R. Morowitz, J. Piepmeier, W. Hait, T. Byrne, M. Salter, J. Imperato, J. Khandekar, N. Paleologos, P. Burger, G.C. Bentel, A. Friedman (1996) A phase III randomized prospective trial of external beam radiotherapy, mitomycin $C$, carmustine, and 6-mercaptopurine for the treatment of adults with anaplastic glioma of the brain. CNS Cancer Consortium. Int J Radiat Oncol Biol Phys. 34(4): 793-802.

60. Happold, C., P. Roth, W. Wick, N. Schmidt, A.M. Florea, M. Silginer, G. Reifenberger, M. Weller Distinct molecular mechanisms of acquired resistance to temozolomide in glioblastoma cells. Journal of Neurochemistry.

61. Harati, V., R. Khayati, A. Farzan (2011) Fully automated tumor segmentation based on improved fuzzy connectedness algorithm in brain MR images. Computers in Biology and Medicine. 41(7): 483-92.

62. Hart, M.G., R. Grant, R. Garside, G. Rogers, M. Somerville, K. Stein (2008) Temozolomide for high grade glioma. Cochrane Database Syst $\operatorname{Rev}(4)$ : CD007415.

63. Hegi, M.E., L. Liu, J.G. Herman, R. Stupp, W. Wick, M. Weller, M.P. Mehta, M.R. Gilbert (2008) Correlation of O6-methylguanine methyltransferase (MGMT) promoter methylation with clinical outcomes in glioblastoma and clinical strategies to modulate MGMT activity. J Clin Oncol. 26(25): 418999.

64. Hein, P.A., C.J. Eskey, J.F. Dunn, E.B. Hug (2004) Diffusion-weighted imaging in the follow-up of treated high-grade gliomas: tumor recurrence versus radiation injury. AJNR American Journal of Neuroradiology. 25(2): 201-9.

65. Henson, J.W., S. Ulmer, G.J. Harris (2008) Brain tumor imaging in clinical trials. AJNR American Journal of Neuroradiology. 29(3): 419-424.

66. Hoffman, W.F., V.A. Levin, C.B. Wilson (1979) Evaluation of malignant glioma patients during the postirradiation period. J Neurosurg. 50(5): 624-8.

67. Houillier, C., J. Lejeune, A. Benouaich-Amiel, F. Laigle-Donadey, E. Criniere, K. Mokhtari, J. Thillet, J.Y. Delattre, K. Hoang-Xuan, M. Sanson 
(2006) Prognostic impact of molecular markers in a series of 220 primary glioblastomas. Cancer. 106(10): 2218-23.

68. Hu, L.S., L.C. Baxter, K.A. Smith, B.G. Feuerstein, J.P. Karis, J.M. Eschbacher, S.W. Coons, P. Nakaji, R.F. Yeh, J. Debbins, J.E. Heiserman (2009) Relative cerebral blood volume values to differentiate high-grade glioma recurrence from posttreatment radiation effect: direct correlation between image-guided tissue histopathology and localized dynamic susceptibility-weighted contrast-enhanced perfusion MR imaging measurements. AJNR American Journal of Neuroradiology. 30(3): 552-8.

69. Hubner, K.F., J.T. Purvis, S.M. Mahaley, Jr., J.T. Robertson, S. Rogers, W.D. Gibbs, P. King, C.L. Partain (1982) Brain tumor imaging by positron emission computed tomography using 11C-labeled amino acids. Journal of Computer Assisted Tomography. 6(3): 544-50.

70. Ibanez, L., W. Schroeder, L. Ng, J. Cates. The ITK Software Guide. Second Edition. 2005; Available from: http://www.itk.org/ltkSoftwareGuide.pdf.

71. Iliadis, G., P. Selviaridis, A. Kalogera-Fountzila, A. Fragkoulidi, D. Baltas, N. Tselis, A. Chatzisotiriou, D. Misailidou, N. Zamboglou, G. Fountzilas (2009) The importance of tumor volume in the prognosis of patients with glioblastoma: comparison of computerized volumetry and geometric models. Strahlentherapie und Onkologie. 185(11): 743-750.

72. Isselbacher, K.J. (1972) Sugar and amino acid transport by cells in culture-differences between normal and malignant cells. The New England Journal of Medicine. 286(17): 929-933.

73. Kassner, A., R.E. Thornhill (2010) Texture analysis: a review of neurologic MR imaging applications. AJNR American Journal of Neuroradiology. 31(5): 809-816.

74. Kaus, M.R., S.K. Warfield, A. Nabavi, P.M. Black, F.A. Jolesz, R. Kikinis (2001) Automated segmentation of MR images of brain tumors. Radiology. 218(2): 586-91.

75. Kleihues, P., P.C. Burger, B.W. Scheithauer (1993) The new WHO classification of brain tumours. Brain Pathol. 3(3): 255-68.

76. Kleihues, P., L.H. Sobin (2000) World Health Organization classification of tumors. Cancer. 88(12): 2887.

77. Kortmann, R.D. (2011) Strahlentherapie bei Hirngliomen im Erwachsenenalter. Der Onkologe. 17: 37-43.

78. Koukourakis, G.V., V. Kouloulias, G. Zacharias, C. Papadimitriou, P. Pantelakos, G. Maravelis, A. Fotineas, I. Beli, D. Chaldeopoulos, J. Kouvaris (2009) Temozolomide with radiation therapy in high grade brain 
gliomas: pharmaceuticals considerations and efficacy; a review article. Molecules. 14(4): 1561-77.

79. Kreisl, T.N., L. Kim, K. Moore, P. Duic, C. Royce, I. Stroud, N. Garren, M. Mackey, J.A. Butman, K. Camphausen, J. Park, P.S. Albert, H.A. Fine (2009) Phase II trial of single-agent bevacizumab followed by bevacizumab plus irinotecan at tumor progression in recurrent glioblastoma. Journal of Clinical Oncology (JCO). 27(5): 740-5.

80. Krex, D., B. Klink, C. Hartmann, A. von Deimling, T. Pietsch, M. Simon, M. Sabel, J.P. Steinbach, O. Heese, G. Reifenberger, M. Weller, G. Schackert (2007) Long-term survival with glioblastoma multiforme. Brain. 130(Pt 10): 2596-606.

81. Kumar, A.J., N.E. Leeds, G.N. Fuller, P. Van Tassel, M.H. Maor, R.E. Sawaya, V.A. Levin (2000) Malignant gliomas: MR imaging spectrum of radiation therapy-and chemotherapy-induced necrosis of the brain after treatment. Radiology. 217(2): 377-84.

82. Kumar, M., K. Mehta (2011) A Modified Method to Segment Sharp and Unsharp Edged Brain Tumors in 2 D MRI Using Automatic Seeded Region Growing Method. International Journal of Soft Computing and Engineering (IJSCE). 1(2): 37-40.

83. la Fougere, C., B. Suchorska, P. Bartenstein, F.W. Kreth, J.C. Tonn (2011) Molecular imaging of gliomas with PET: opportunities and limitations. Neuro-Oncology. 13(8): 806-819.

84. Lacroix, M., D. Abi-Said, D.R. Fourney, Z.L. Gokaslan, W. Shi, F. DeMonte, F.F. Lang, I.E. McCutcheon, S.J. Hassenbusch, E. Holland, K. Hess, C. Michael, D. Miller, R. Sawaya (2001) A multivariate analysis of 416 patients with glioblastoma multiforme: prognosis, extent of resection, and survival. J Neurosurg. 95(2): 190-8.

85. Langen, K.J., M. Jarosch, H. Muhlensiepen, K. Hamacher, S. Broer, P. Jansen, K. Zilles, H.H. Coenen (2003) Comparison of fluorotyrosines and methionine uptake in F98 rat gliomas. Nuclear Medicine and Biology. 30(5): 501-8.

86. Laprie, A. (2009) [Proton magnetic resonance spectroscopic imaging and other types of metabolic imaging for radiotherapy planning in adult and pediatric high-grade gliomas]. Cancer Radiother. 13(6-7): 556-61.

87. Le Jeune, F.P., F. Dubois, S. Blond, M. Steinling (2006) Sestamibi technetium-99m brain single-photon emission computed tomography to identify recurrent glioma in adults: 201 studies. Journal of Neuro-Oncology. 77(2): 177-183. 
88. Lee, C.H., S. Wang, A. Murtha, M.R. Brown, R. Greiner. Segmenting brain tumors using pseudo-conditional random fields. in Medical Image Computing and Computer-Assisted Intervention - MICCAI 2008. 2008. New York, NY, USA.

89. Lerski, R.A., K. Straughan, L.R. Schad, D. Boyce, S. Bluml, I. Zuna (1993) MR image texture analysis--an approach to tissue characterization. Magn Reson Imaging. 11(6): 873-87.

90. Lin, I., H. Chao. CMAS: a rich media annotation system for medical imaging. in Medical Imaging 2006: PACS and Imaging Informatics. 2006. San Diego, CA, USA: SPIE.

91. Louis, D., D. Schiff, T. Batchelor. Classification of gliomas. 2012; Available from: www.uptodate.com.

92. Louis, D.N. (2006) Molecular pathology of malignant gliomas. Annu Rev Pathol. 1: 97-117.

93. Louis, D.N., H. Ohgaki, O.D. Wiestler, W.K. Cavenee, P.C. Burger, A. Jouvet, B.W. Scheithauer, P. Kleihues (2007) The 2007 WHO classification of tumours of the central nervous system. Acta Neuropathol. 114(2): 97109.

94. Louis, D.N., S.L. Pomeroy, J.G. Cairncross (2002) Focus on central nervous system neoplasia. Cancer Cell. 1(2): 125-8.

95. Macdonald, D.R., T.L. Cascino, S.C. Schold, Jr., J.G. Cairncross (1990) Response criteria for phase II studies of supratentorial malignant glioma. J Clin Oncol. 8(7): 1277-80.

96. Macdonald, D.R., T.L. Cascino, S.C. Schold, Jr., J.G. Cairncross (1990) Response criteria for phase II studies of supratentorial malignant glioma. Journal of Clinical Oncology (JCO). 8(7): 1277-80.

97. Mangla, R., G. Singh, D. Ziegelitz, M.T. Milano, D.N. Korones, J. Zhong, S.E. Ekholm (2010) Changes in relative cerebral blood volume 1 month after radiation-temozolomide therapy can help predict overall survival in patients with glioblastoma. Radiology. 256(2): 575-84.

98. Mazzara, G.P., R.P. Velthuizen, J.L. Pearlman, H.M. Greenberg, H. Wagner (2004) Brain tumor target volume determination for radiation treatment planning through automated MRI segmentation. Int J Radiat Oncol Biol Phys. 59(1): 300-12.

99. Mechouche, A., X. Morandi, C. Golbreich, B. Gibaud. A hybrid system for the semantic annotation of sulco-gyral anatomy in MRI images. in 11th International Conference in Medical Image Computing and ComputerAssisted Intervention - MICCAI 2008. 2008. New York, NY, USA. 
100. Mehrkens, J.H., G. Popperl, W. Rachinger, J. Herms, K. Seelos, K. Tatsch, J.C. Tonn, F.W. Kreth (2008) The positive predictive value of O-(2[18F]fluoroethyl)-L-tyrosine (FET) PET in the diagnosis of a glioma recurrence after multimodal treatment. Journal of Neuro-Oncology. 88(1): 27-35.

101. Miller, A.B., B. Hoogstraten, M. Staquet, A. Winkler (1981) Reporting results of cancer treatment. Cancer. 47(1): 207-14.

102. Mohd Saad, N., S.A.R. Abi-Bakar, S. Muda, M. Mokji, A.R. Abdullah. Automated Region Growing for Segmentation of Brain Lesion in Diffusionweighted MRI. in International MultiConference of Engineers and Computer Scientists 2012 (IMECS 2012). 2012. Hong Kong.

103. Najafi, M., H. Soltanian-Zadeh, K. Jafari-Khouzani, L. Scarpace, T. Mikkelsen (2012) Prediction of glioblastoma multiform response to bevacizumab treatment using multi-parametric MRI. PLoS One. 7(1): e29945.

104. Nakajima, T., T. Kumabe, M. Kanamori, R. Saito, M. Tashiro, M. Watanabe, T. Tominaga (2009) Differential diagnosis between radiation necrosis and glioma progression using sequential proton magnetic resonance spectroscopy and methionine positron emission tomography. Neurologia medico-chirurgica (Tokyo). 49(9): 394-401.

105. Norden, A.D., J. Drappatz, A. Muzikansky, K. David, M. Gerard, M.B. McNamara, P. Phan, A. Ross, S. Kesari, P.Y. Wen (2009) An exploratory survival analysis of anti-angiogenic therapy for recurrent malignant glioma. Journal of Neuro-Oncology. 92(2): 149-55.

106. Noushmehr, H., D.J. Weisenberger, K. Diefes, H.S. Phillips, K. Pujara, B.P. Berman, F. Pan, C.E. Pelloski, E.P. Sulman, K.P. Bhat, R.G. Verhaak, K.A. Hoadley, D.N. Hayes, C.M. Perou, H.K. Schmidt, L. Ding, R.K. Wilson, D. Van Den Berg, H. Shen, H. Bengtsson, P. Neuvial, L.M. Cope, J. Buckley, J.G. Herman, S.B. Baylin, P.W. Laird, K. Aldape Identification of a CpG island methylator phenotype that defines a distinct subgroup of glioma. Cancer Cell. 17(5): 510-22.

107. Payer, F. (2011) [Pseudoprogression or pseudoresponse: a challenge for the diagnostic imaging in Glioblastoma multiforme]. Wiener Medizinische Wochenschrift. 161(1-2): 13-19.

108. Pednekar, A.S., I.A. Kakadiaris (2006) Image segmentation based on fuzzy connectedness using dynamic weights. IEEE Trans Image Process. 15(6): 1555-62.

109. Peng, H., F. Long, E.W. Myers (2009) VANO: a volume-object image annotation system. Bioinformatics. 25(5): 695-7. 
110. Perry, A., R.E. Schmidt (2006) Cancer therapy-associated CNS neuropathology: an update and review of the literature. Acta Neuropathologica. 111(3): 197-212.

111. Pichlmeier, U., A. Bink, G. Schackert, W. Stummer (2008) Resection and survival in glioblastoma multiforme: an RTOG recursive partitioning analysis of ALA study patients. Neuro Oncol. 10(6): 1025-34.

112. Plotkin, M., J. Eisenacher, H. Bruhn, R. Wurm, R. Michel, F. Stockhammer, A. Feussner, O. Dudeck, P. Wust, R. Felix, H. Amthauer (2004) 123I-IMT SPECT and $1 H$ MR-spectroscopy at $3.0 \mathrm{~T}$ in the differential diagnosis of recurrent or residual gliomas: a comparative study. Journal of NeuroOncology. 70(1): 49-58.

113. Pope, W.B., X.J. Qiao, H.J. Kim, A. Lai, P. Nghiemphu, X. Xue, B.M. Ellingson, D. Schiff, D. Aregawi, S. Cha, V.K. Puduvalli, J. Wu, W.K. Yung, G.S. Young, J. Vredenburgh, D. Barboriak, L.E. Abrey, T. Mikkelsen, R. Jain, N.A. Paleologos, P.L. Rn, M. Prados, J. Goldin, P.Y. Wen, T. Cloughesy (2012) Apparent diffusion coefficient histogram analysis stratifies progression-free and overall survival in patients with recurrent GBM treated with bevacizumab: a multi-center study. Journal of Neuro-Oncology. 108(3): 491-498.

114. Pöpperl, G., C. Gotz, W. Rachinger, O. Schnell, F.J. Gildehaus, J.C. Tonn, K. Tatsch (2006) Serial O-(2-[(18)F]fluoroethyl)-L: -tyrosine PET for monitoring the effects of intracavitary radioimmunotherapy in patients with malignant glioma. European Journal of Nuclear Medicine and Molecular Imaging. 33(7): 792-800.

115. Rachinger, W., C. Goetz, G. Popperl, F.J. Gildehaus, F.W. Kreth, M. Holtmannspotter, J. Herms, W. Koch, K. Tatsch, J.C. Tonn (2005) Positron emission tomography with O-(2-[18F]fluoroethyl)-l-tyrosine versus magnetic resonance imaging in the diagnosis of recurrent gliomas. Neurosurgery. 57(3): 505-511.

116. Rees, J. (2002) Glioma Therapy. ANCR. 2(2): 11.

117. Reiser, M., F.-P. Kuhn, J. Debus (2011) Duale Reihe Radiologie. 3rd ed: Thieme, Stuttgart.

118. Ringel, F., J. Gempt, N. Buchmann, S. Krieg, H. Pape, E. Shiban, Y.M. Ryang, B. Meyer (2011) Stellenwert der Tumorresektion in der interdisziplinären Behandlung hirneigener Tumore. Der Onkologe. 17: 31 36.

119. Roelcke, U., E.W. Radu, K. von Ammon, O. Hausmann, R.P. Maguire, K.L. Leenders (1995) Alteration of blood-brain barrier in human brain tumors: comparison of [18F]fluorodeoxyglucose, [11C]methionine and rubidium-82 
using PET. Journal of the Neurological Sciences. 132(1): 20-27.

120. Rosenfeld, A. (1970) Connectivity in digital pictures. Journal of the Association for Computing Machinery (JACM). 17(1): 146-160.

121. Ruben, J.D., M. Dally, M. Bailey, R. Smith, C.A. McLean, P. Fedele (2006) Cerebral radiation necrosis: incidence, outcomes, and risk factors with emphasis on radiation parameters and chemotherapy. International Journal of Radiation Oncology • Biology • Physics. 65(2): 499-508.

122. Sasajima, T., T. Miyagawa, T. Oku, J.G. Gelovani, R. Finn, R. Blasberg (2004) Proliferation-dependent changes in amino acid transport and glucose metabolism in glioma cell lines. European Journal of Nuclear Medicine and Molecular Imaging. 31(9): 1244-1256.

123. Scott, J.N., P.M. Brasher, R.J. Sevick, N.B. Rewcastle, P.A. Forsyth (2002) How often are nonenhancing supratentorial gliomas malignant? A population study. Neurology. 59(6): 947-9.

124. Shah, G.D., S. Kesari, R. Xu, T.T. Batchelor, A.M. O'Neill, F.H. Hochberg, B. Levy, J. Bradshaw, P.Y. Wen (2006) Comparison of linear and volumetric criteria in assessing tumor response in adult high-grade gliomas. Neuro Oncol. 8(1): 38-46.

125. Shih, H.A., T. Batchelor. Adjuvant radiation therapy for malignant gliomas. 2012; Available from: www.uptodate.com.

126. Skounakis, E., C. Farmaki, V. Sakkalis, A. Roniotis, K. Banitsas, N. Graf, K. Marias (2010) DoctorEye: A clinically driven multifunctional platform, for accurate processing of tumors in medical images. The Open Medical Informatics Journal. 4: 105-115.

127. Skounakis, E., V. Sakkalis, K. Marias, K. Banitsas, N. Graf. DoctorEye: A multifunctional open platform for fast annotation and visualization of tumors in medical images. in 31st Annual international conference of the IEEE Engineering in Medicine and Biology Society. 2009. Minneapolis, Minnesota, USA.

128. Skounakis, E., V. Sakkalis, K. Marias, K. Banitsas, N. Graf, DoctorEye: A multifunctional open platform for fast annotation and visualization of tumors in medical images, in Engineering in Medicine and Biology Society, 2009. EMBC 2009. Annual International Conference of the IEEE. 2009: Minneapolis, Minnesota. p. 3759-3762.

129. Sorensen, A.G., T.T. Batchelor, P.Y. Wen, W.T. Zhang, R.K. Jain (2008) Response criteria for glioma. Nature Clinical Practice Oncology. 5(11): 634644.

130. Stamatakos, G.S., V.P. Antipas, N.K. Uzunoglu (2006) A spatiotemporal, 
patient individualized simulation model of solid tumor response to chemotherapy in vivo: the paradigm of glioblastoma multiforme treated by temozolomide. IEEE Transactions on Biomedical Engineering. 53(8): 14671477.

131. Stamatakos, G.S., D.D. Dionysiou, N.M. Graf, N.A. Sofra, C. Desmedt, A. Hoppe, N.K. Uzunoglu, M. Tsiknakis (2007) The "Oncosimulator": a multilevel, clinically oriented simulation system of tumor growth and organism response to therapeutic schemes. Towards the clinical evaluation of in silico oncology. Conf Proc IEEE Eng Med Biol Soc. 2007: 6629-32.

132. Stummer, W., U. Pichlmeier, T. Meinel, O.D. Wiestler, F. Zanella, H.J. Reulen (2006) Fluorescence-guided surgery with 5-aminolevulinic acid for resection of malignant glioma: a randomised controlled multicentre phase III trial. Lancet Oncol. 7(5): 392-401.

133. Stummer, W., H.J. Reulen, T. Meinel, U. Pichlmeier, W. Schumacher, J.C. Tonn, V. Rohde, F. Oppel, B. Turowski, C. Woiciechowsky, K. Franz, T. Pietsch (2008) Extent of resection and survival in glioblastoma multiforme: identification of and adjustment for bias. Neurosurgery. 62(3): 564-76; discussion 564-76.

134. Stupp, R., W.P. Mason, M.J. van den Bent, M. Weller, B. Fisher, M.J. Taphoorn, K. Belanger, A.A. Brandes, C. Marosi, U. Bogdahn, J. Curschmann, R.C. Janzer, S.K. Ludwin, T. Gorlia, A. Allgeier, D. Lacombe, J.G. Cairncross, E. Eisenhauer, R.O. Mirimanoff (2005) Radiotherapy plus concomitant and adjuvant temozolomide for glioblastoma. N Engl J Med. 352(10): 987-96.

135. Sun, D., Q. Liu, W. Liu, W. Hu (2000) Clinical application of 201TI SPECT imaging of brain tumors. The Journal of Nuclear Medicine. 41(1): 5-10.

136. Taal, W., D. Brandsma, H.G. de Bruin, J.E. Bromberg, A.T. Swaak-Kragten, P.A. Smitt, C.A. van Es, M.J. van den Bent (2008) Incidence of early pseudo-progression in a cohort of malignant glioma patients treated with chemoirradiation with temozolomide. Cancer. 113(2): 405-10.

137. Terakawa, Y., N. Tsuyuguchi, Y. Iwai, K. Yamanaka, S. Higashiyama, T. Takami, K. Ohata (2008) Diagnostic accuracy of 11C-methionine PET for differentiation of recurrent brain tumors from radiation necrosis after radiotherapy. The Journal of Nuclear Medicine. 49(5): 694-9.

138. Therasse, P., S.G. Arbuck, E.A. Eisenhauer, J. Wanders, R.S. Kaplan, L. Rubinstein, J. Verweij, M. Van Glabbeke, A.T. van Oosterom, M.C. Christian, S.G. Gwyther (2000) New guidelines to evaluate the response to treatment in solid tumors. European Organization for Research and Treatment of Cancer, National Cancer Institute of the United States, National Cancer Institute of Canada. J Natl Cancer Inst. 92(3): 205-16. 
139. Tie, J., D.H. Gunawardana, M.A. Rosenthal (2008) Differentiation of tumor recurrence from radiation necrosis in high-grade gliomas using $201 \mathrm{TI}$ SPECT. Journal of Clinical Neuroscience. 15(12): 1327-34.

140. Tourassi, G.D. (1999) Journey toward computer-aided diagnosis: role of image texture analysis. Radiology. 213(2): 317-320.

141. Tsien, C., C.J. Galban, T.L. Chenevert, T.D. Johnson, D.A. Hamstra, P.C. Sundgren, L. Junck, C.R. Meyer, A. Rehemtulla, T. Lawrence, B.D. Ross (2010) Parametric response map as an imaging biomarker to distinguish progression from pseudoprogression in high-grade glioma. Journal of Clinical Oncology (JCO). 28(13): 2293-9.

142. Udupa, J.K., S. Samarasekera (1996) Fuzzy Connectedness and Object Definition: Theory, Algorithms, and Applications in Image Segmentation. Graphical Models and Image Processing. 58(3): 246-261.

143. Ueki, K., Y. Ono, J.W. Henson, J.T. Efird, A. von Deimling, D.N. Louis (1996) CDKN2/p16 or RB alterations occur in the majority of glioblastomas and are inversely correlated. Cancer Res. 56(1): 150-3.

144. Ullrich, R.T., L. Kracht, A. Brunn, K. Herholz, P. Frommolt, H. Miletic, M. Deckert, W.D. Heiss, A.H. Jacobs (2009) Methyl-L-11C-methionine PET as a diagnostic marker for malignant progression in patients with glioma. The Journal of Nuclear Medicine. 50(12): 1962-1968.

145. Vaidyanathan, M., L.P. Clarke, R.P. Velthuizen, S. Phuphanich, A.M. Bensaid, L.O. Hall, J.C. Bezdek, H. Greenberg, A. Trotti, M. Silbiger (1995) Comparison of supervised MRI segmentation methods for tumor volume determination during therapy. Magn Reson Imaging. 13(5): 719-28.

146. Van Laere, K., S. Ceyssens, F. Van Calenbergh, T. de Groot, J. Menten, P. Flamen, G. Bormans, L. Mortelmans (2005) Direct comparison of 18F-FDG and 11C-methionine PET in suspected recurrence of glioma: sensitivity, inter-observer variability and prognostic value. European Journal of Nuclear Medicine and Molecular Imaging. 32(1): 39-51.

147. Verhaak, R.G., K.A. Hoadley, E. Purdom, V. Wang, Y. Qi, M.D. Wilkerson, C.R. Miller, L. Ding, T. Golub, J.P. Mesirov, G. Alexe, M. Lawrence, M. O'Kelly, P. Tamayo, B.A. Weir, S. Gabriel, W. Winckler, S. Gupta, L. Jakkula, H.S. Feiler, J.G. Hodgson, C.D. James, J.N. Sarkaria, C. Brennan, A. Kahn, P.T. Spellman, R.K. Wilson, T.P. Speed, J.W. Gray, M. Meyerson, G. Getz, C.M. Perou, D.N. Hayes Integrated genomic analysis identifies clinically relevant subtypes of glioblastoma characterized by abnormalities in PDGFRA, IDH1, EGFR, and NF1. Cancer Cell. 17(1): 98-110.

148. Vredenburgh, J.J., A. Desjardins, J.E. Herndon, 2nd, J. Marcello, D.A. Reardon, J.A. Quinn, J.N. Rich, S. Sathornsumetee, S. Gururangan, J. 
Sampson, M. Wagner, L. Bailey, D.D. Bigner, A.H. Friedman, H.S. Friedman (2007) Bevacizumab plus irinotecan in recurrent glioblastoma multiforme. Journal of Clinical Oncology (JCO). 25(30): 4722-9.

149. Wang, M.Y., J.L. Cheng, Y.H. Han, Y.L. Li, J.P. Dai, D.P. Shi (2012) Measurement of tumor size in adult glioblastoma: classical cross-sectional criteria on 2D MRI or volumetric criteria on high resolution $3 D \mathrm{MRI}$ ? European Journal of Radiology. 81(9): 2370-2374.

150. Wang, Z., T. Deisboeck (2008) Computational modeling of brain tumors: discrete, continuum or hybrid? Scientific Modeling and Simulation SMNS. 15(1-3): 381-393.

151. Warmuth-Metz, M. (2011) Neuroradiologie bei Gliomen und Metastasen. Der Onkologe. 17: 18-30.

152. Warren, K.E., N. Patronas, A.A. Aikin, P.S. Albert, F.M. Balis (2001) Comparison of one-, two-, and three-dimensional measurements of childhood brain tumors. J Natl Cancer Inst. 93(18): 1401-5.

153. Weber, W.A., A.L. Grosu (2011) PET bei Hirntumoren. Der Onkologe. 17(4): 318-328.

154. Wels, M., G. Carneiro, A. Aplas, M. Huber, J. Hornegger, D. Comaniciu. A discriminative model-constrained graph cuts approach to fully automated pediatric brain tumor segmentation in 3-D MRI. in Medical Image Computing and Computer-Assisted Intervention - MICCAI 2008. 2008.

155. Weltens, C., J. Menten, M. Feron, E. Bellon, P. Demaerel, F. Maes, W. Van den Bogaert, E. van der Schueren (2001) Interobserver variations in gross tumor volume delineation of brain tumors on computed tomography and impact of magnetic resonance imaging. Radiother Oncol. 60(1): 49-59.

156. Wen, P.Y. Assessment of disease status and surveillance after treatment in patients with brain tumors. 2011; Available from: www.uptodate.com.

157. Wen, P.Y., S. Kesari (2008) Malignant gliomas in adults. N Engl J Med. 359(5): 492-507.

158. Wen, P.Y., D.R. Macdonald, D.A. Reardon, T.F. Cloughesy, A.G. Sorensen, E. Galanis, J. Degroot, W. Wick, M.R. Gilbert, A.B. Lassman, C. Tsien, T. Mikkelsen, E.T. Wong, M.C. Chamberlain, R. Stupp, K.R. Lamborn, M.A. Vogelbaum, M.J. van den Bent, S.M. Chang (2010) Updated response assessment criteria for high-grade gliomas: response assessment in neurooncology working group. J Clin Oncol. 28(11): 1963-72.

159. Wen, P.Y., D.R. Macdonald, D.A. Reardon, T.F. Cloughesy, A.G. Sorensen, E. Galanis, J. Degroot, W. Wick, M.R. Gilbert, A.B. Lassman, C. Tsien, T. Mikkelsen, E.T. Wong, M.C. Chamberlain, R. Stupp, K.R. Lamborn, M.A. 
Vogelbaum, M.J. van den Bent, S.M. Chang (2010) Updated response assessment criteria for high-grade gliomas: response assessment in neurooncology working group. Journal of Clinical Oncology (JCO). 28(11): 196372.

160. Wick, W., M. Weller, M. van den Bent, R. Stupp (2010) Bevacizumab and recurrent malignant gliomas: a European perspective. Journal of Clinical Oncology (JCO). 28(12): e188-9; author reply e190-2.

161. Wong, E.T., J.K. Wu. Clinical presentation and diagnosis of brain tumors. 2010; Available from: www.uptodate.com.

162. Würker, M., K. Herholz, J. Voges, U. Pietrzyk, H. Treuer, B. Bauer, V. Sturm, W.D. Heiss (1996) Glucose consumption and methionine uptake in low-grade gliomas after iodine-125 brachytherapy. European Journal of Nuclear Medicine. 23(5): 583-6.

163. Xue, D., R.E. Albright, Jr. (1999) Preoperative anaplastic glioma tumor volume effects on patient survival. Journal of Surgical Oncology. 72(4): 199205.

164. Yamamoto, Y., Y. Nishiyama, Y. Toyama, K. Kunishio, K. Satoh, M. Ohkawa (2002) 99mTc-MIBI and 201TI SPET in the detection of recurrent brain tumours after radiation therapy. Nuclear Medicine Communications. 23(12): 1183-1190.

165. Yan, H., D.W. Parsons, G. Jin, R. McLendon, B.A. Rasheed, W. Yuan, I. Kos, I. Batinic-Haberle, S. Jones, G.J. Riggins, H. Friedman, A. Friedman, D. Reardon, J. Herndon, K.W. Kinzler, V.E. Velculescu, B. Vogelstein, D.D. Bigner (2009) IDH1 and IDH2 mutations in gliomas. N Engl J Med. 360(8): 765-73.

166. Yong, W.H., D. Chou, K. Ueki, G.R.t. Harsh, A. von Deimling, J.F. Gusella, H.W. Mohrenweiser, D.N. Louis (1995) Chromosome 19q deletions in human gliomas overlap telomeric to D19S219 and may target a $425 \mathrm{~kb}$ region centromeric to D19S112. J Neuropathol Exp Neurol. 54(5): 622-6.

167. Yu, W., L. Williams, E. Malveaux, V.M. Camp, J.J. Olson, M.M. Goodman (2008) Synthesis and evaluation of [123l] labeled iodovinyl amino acids syn-, anti-1-amino-3-[2-iodoethenyl]-cyclobutane-1-carboxylic acid, and 1amino-3-iodomethylene-cyclobutane-1-carboxylic acid as potential SPECT brain tumor imaging agents. Bioorganic \& Medicinal Chemistry Letters. 18(4): 1264-1268.

168. Zeng, Q.S., C.F. Li, K. Zhang, H. Liu, X.S. Kang, J.H. Zhen (2007) Multivoxel 3D proton MR spectroscopy in the distinction of recurrent glioma from radiation injury. Journal of Neuro-Oncology. 84(1): 63-9. 
169. Zhang, L., Z. Wang, J.A. Sagotsky, T.S. Deisboeck (2009) Multiscale agentbased cancer modeling. Journal of Mathematical Biology. 58(4-5): 545-559.

170. Zulch, K.J. (1980) Principles of the new World Health Organization (WHO) classification of brain tumors. Neuroradiology. 19(2): 59-66. 


\section{Acknowledgment}

An dieser Stelle möchte ich mich ganz herzlich bei allen bedanken, die mich unterstützt und das Gelingen dieser Arbeit ermöglicht haben.

An erster Stelle bedanke ich mich ganz herzlich bei Herrn Prof. Norbert Graf, Direktor der Klinik für Pädiatrische Onkologie und Hämatologie, für die Überlassung des Themas dieser Doktorarbeit und die ausgezeichnete Betreuung bei der Durchführung der Arbeit. Seine freundliche Unterstützung, die wissenschaftlichen Anregungen, aber auch seine Geduld waren meine Triebfeder und schafften ein Arbeitsklima, welches für jeden Doktoranden wünschenswert wäre. Außerdem bedanke ich mich für die Möglichkeit, Teile dieser Arbeit auf einem internationalen Kongress vorzustellen.

Herrn Prof. Wolfgang Reith danke ich für die Bereitstellung der untersuchten BildDaten und seiner fachlichen Kompetenz bei verschiedenen Fragestellungen.

Ich danke Frau Elisabeth Friedel für ihre stets freundliche Hilfsbereitschaft.

Mein Dank gilt auch dem Team der Biomodeling-Group (FORTH, Kreta) um Kostas Marias, für die konstruktive Zusammenarbeit und den beiden EU Projekten ContraCancrum und TUMOR, in deren Rahmen wir unsere Studie durchführten.

Liebe Verena, vielen Dank für deine unermüdliche Geduld, dein Verständnis, deinen Ansporn und deinen wohl dosierten Optimismus, zum Erreichen dieses gemeinsam lange herbeigesehnten Zieles.

Von Herzen gilt mein Dank in besonderem Maße meinen Eltern Fred und Cornelia, aber auch meiner Schwester Charlotte, meinen Großeltern Elfriede, Bütt, Ebs und Willi, und meiner Patentante Wilma, die immer für mich da waren und in jeder Lebenslage ein aufmunterndes Wort gefunden haben. Ohne euch wären mir viele Dinge im Leben sicherlich nicht in dieser Form möglich gewesen.

Ihnen ist diese Arbeit gewidmet. 


\section{Publications}

Nov. 2012

An innovative mathematical analysis of routine MRI scans in patients with glioblastoma using DoctorEye

Jonathan Zepp, Norbert Graf, Ioannis Karatzanis, Holger Stenzhorn, Georgios C. Manikis, Vangelis Sakkalis, Wolfgang Reith, Georgios Stamatakos, Konstantinos Marias

IEEE $12^{\text {th }}$ International Conference on Bioinformatics \& Bioengineering (BIBE 2012), Larnaca, Cyprus, Nov. 11-13, 2012

Sep. 2010

Tumor segmentation: The impact of standardized signal intensity histograms in glioblastoma

Jonathan Zepp, Norbert Graf, Emmanouil Skounakis, Rainer Bohle, Eckart Meese, Holger Stenzhorn, Yoo-Jin Kim, Christina Farmaki, Vangelis Sakkalis, Wolfgang Reith, Georgios Stamatakos, Konstantinos Marias

$4^{\text {th }}$ International Advanced Research Workshop on In Silico Oncology and Cancer Investigation (4th IARWISOCI) - The ContraCancrum Workshop, Athens, Greece, Sept. 8-9, 2010 (www.4th-iarwisoci.iccs.ntua.gr) 


\section{Appendix}

\subsection{List of Figures}

Figure 1: DICOM Data-Set 21

Figure 2: Age Distribution of the Study Group. 23

Figure 3: Siemens 1.5T MAGNETOM Vision MRI Scanner [8].... 26

Figure 4: DoctorEye Webpage 28

Figure 5: DoctorEye Task Bar with the Annotation-Tools "Pencil, Eraser" and the "Magic Wand". 29

Figure 6: Graphical User Interface (GUI) of DoctorEye 4.7. 30

Figure 7: Comparison between raw and processed MR-Image. 31

Figure 8: Histogram calculated by DoctorEye 32

Figure 9: Histogram Data Transfer into Microsoft Office Excel 2007 32

Figure 10: Histogram Analysis in Microsoft Office Word 2007 35

Figure 11: Illustration of DoctorEye's high Potential of precise Delineation. 38

Figure 12: Illustration of the Software's high grade Accuracy in an amplified Image 39

Figure 13: 3D-Vizualization created by DoctorEye 40

Figure 14: Different Sequences of $M R$ Images in a single Patient with Glioblastoma at the Time of Diagnosis.

Figure 15: Exemplary annotated DICOM Files of all Sequences from Patient G-13 at the Time of Diagnosis with corresponding Histograms of the whole Set. 43 
Figure 16: Mean Histogram calculated from all Patients at the Time of Diagnosis 45

Figure 17: Follow-up of Patient G-25 in T1-Sequence 48

Figure 18: Follow-up of Patient G-25 in T1 with Gadolinium Enhancement Sequence 50

Figure 19: Follow-up of Patient G-25 in T2 Sequence. 52

Figure 20: Follow-up of Patient G-25 in T2 Flair Sequence. 54

Figure 21: Mean Histogram from T1-Sequence during Follow-up 56

Figure 22: Mean Histogram from T1 with Gadolinium Contrast Enhancement during Follow-up. 58

Figure 23: Mean Histogram from T2-Sequence during Follow-up 60

Figure 24: Mean Histogram from T2 Flair-Sequence during Follow-up 62

Figure 25: Follow-up of Patient G-8 in T1 with Gadolinium Contrast Enhancement Sequence 64

Figure 26: Volume Changes during Follow-up in Patient G-25 for all Sequences. Comparison of the whole Lesion to the three Subunits (Active Tumor, Necrosis and Edema). 68

Figure 27: Volume Changes during Follow-up in the Summary, for all Sequences. Comparison of the whole Lesion to the three Subunits (Active Tumor, Necrosis and Edema). 69

Figure 28: Volume Development during different Time points in a Patient that suffers from real Tumor Progression after receiving Surgery and Radiation- and Chemotherapy (G-17) 71

Figure 29: Volume Development during different Time Points in a Patient that shows questionable Progression after receiving Surgery and Radiation- and 
Chemotherapy (G-12) 72

Figure 30: Volume Development during different Time Points in a Patient that shows stable Disease after receiving Surgery and Radiation- and Chemotherapy (G-7)

Figure 31: Volume Development during different Time Points in a Patient that shows questionable Response after receiving Surgery and Radiation- and Chemotherapy (G-23)

Figure 32: Development of SI-Peak during Follow-up in a Patient that suffers from Tumor Recurrence (G-17)

Figure 33: Development of Mean-SI during Follow-up in a Patient that suffers from Tumor Recurrence (G-17) 88

Figure 34: Development of Standardized-SI during Follow-up in a Patient that suffers from Tumor Recurrence (G-17) 89

Figure 35: Development of SI-Peak during Follow-up in a Patient with stable Disease (G-7) . 90

Figure 36: Development of Mean-SI during Follow-up in a Patient with stable Disease (G-7) 91

Figure 37: Development of Standardized SI during Follow-up in a Patient with stable Disease (G-7) 92

Figure 38: Ethical Approval from the 'Ärztekammer des Saarlandes' 134

\subsection{List of Tables}

Table I. Current Response Criteria for Malignant Gliomas 16 
Table II. Study Group - Date of Diagnosis, Histology and WHO grading..... 22

Table III: Exemplary Excel spreadsheet demonstrating the mathematical Analysis of Signalintensity Histograms

Table IV: Results from mathematical Analysis of the mean Histograms at the Time of Diagnosis. 46

Table V: Results from mathematical Analysis of Patient G-25's Histograms from T1-Sequence during Follow-up

Table VI: Results from mathematical Analysis of Patient G-25's Histograms from T1 with Gadolinium Enhancement Sequence during Follow-up 51

Table VII: Results from mathematical Analysis of Patient G-25's Histograms from T2-Sequence during Follow-up 53

Table VIII: Results from mathematical Analysis of Patient G-25's Histograms from T2 Flair-Sequence during Follow-up 55

Table IX: Results from mathematical Analysis of the mean Histograms from T1Sequence during Follow-up 57

Table X: Results from mathematical Analysis of the mean Histograms from T1 with Gadolinium Contrast Enhancement during Follow-up 59

Table XI: Results from mathematical Analysis of the mean Histograms from T2Sequence during Follow-up 61

Table XII: Results from mathematical Analysis of the mean Histograms from T2 Flair-Sequence during Follow-up. 63

Table XIII: Results from mathematical Analysis of Patient G-8 from T1 with Gadolinium Contrast Enhancement during Follow-up. 65

Table XIV: SI-Peak of all Sequences at different Time Points calculated from all Patients of this Series. 66 
Table XV: Mean Signalintensity of all Sequences at different Time Points calculated from all Patients of this Series 66

Table XVI: Standardized mean SI of all Sequences at different Time Points calculated from all Patients of this Series 67

\subsection{Abbreviations}

In addition to the abbreviations of the English spelling dictionary and the international units, the following abbreviations have been used:

$\left[{ }^{11} \mathrm{C}\right] \mathrm{MET} \quad$ Methionine

$\left[{ }^{18} \mathrm{~F}\right] \mathrm{FDG} \quad$ Fluorodeoxyglucose

$\left[{ }^{18} \mathrm{~F}\right] \mathrm{FET} \quad$ Fluorethyltyrosin

$\left[{ }^{18} \mathrm{~F}\right] \mathrm{FLT} \quad$ Fluorothymidine

${ }^{123}$ I $\quad{ }^{123}$ Iodine-Alpha-Methyl-Tyrosine

${ }^{201} \mathrm{TI} \quad{ }^{201}$ Thallium

${ }^{99 m}$ Tc $\quad{ }^{99 m}$ Technetium-Glucoheptonic-Acid

${ }^{99 m}$ Tc-MIBI ${ }^{99 m}$ Tchexakis-2-Methoxyisobutylisonitrile

ADC Apparent Diffusion Coefficient

ADC-L $\quad$ ADC from the Lower curve

BCNU Bis-Chloroethyl-Nitrosourea

CCNU Chemotherapy Consisting of Lomustine

Cho Choline

CNS Central Nervous System

CPU Central Processing Unit

CR Complete response

CRF Conditional Random Fields

CT Computer Tomography 
DICOM Digital Imaging and Communications in Medicine

DWI Diffusion Weighted Imaging

EGFR Epidermal Growth Factor Receptor

ET Echo Time

EU European Union

FLAIR Fluid Attenuated Inversion Recovery

FORTH Foundation for Research and Technology - Hellas

GBM Glioblastoma Multiforme

Gd Gadolinium

Gd-MRI Gadolinium-enhanced Magnetic Resonance Imaging

GUI Graphical User Interface

Gy $\quad$ Gray

ICD-O International Classification of Diseases for Oncology

ICS Institute of Computer Science, Crete

IFRT Involved Field Radiation Therapy

iid identically distributed

ITK Insight Toolkit for Segmentation and Registration

KG Knowledge-guided

kNN k Nearest Neighbor

LCP Lower Curve Proportion

MGMT $\quad \mathrm{O}^{6}$-Methyl-Guanine-DNA-Methyltransferase

MRI Magnetic Resonance Imaging

MRS Magnetic Resonance Spectroscopy

NAA N-acetylaspartate

NMR Nuclear Magnetic Resonance 


\begin{tabular}{|c|c|}
\hline OS & Overall Survival \\
\hline PD & Progressive Disease \\
\hline PET & Positron Emission Tomography \\
\hline PFS & Progression-free Survival \\
\hline PP & Pseudoprogression \\
\hline PR & Partial Response \\
\hline PR & Pseudoresponse \\
\hline RANO & Response Assessment in Neuro-Oncology \\
\hline rCBV & Relative Cerebral Blood Volume \\
\hline RECIST & Response Evaluation Criteria in Solid Tumors \\
\hline $\mathrm{RN}$ & Radiation Necrosis \\
\hline $\mathrm{rPH}$ & Relative Peak Height \\
\hline RT & Radiation Therapy \\
\hline SD & Stable Disease \\
\hline SI & Signal-Intensity \\
\hline SPECT & Single Photon Emission CT \\
\hline SVM & Support Vector machine classification \\
\hline SWI & Susceptibility-weighted Imaging \\
\hline TMZ & Temozolomide \\
\hline TR & Repetition Time \\
\hline VEGF & Vascular Endothelia Growth Factor \\
\hline WBRT & Whole Brain Radiation Therapy \\
\hline WHO & World Health Organization \\
\hline
\end{tabular}




\subsection{Ethical Approval}

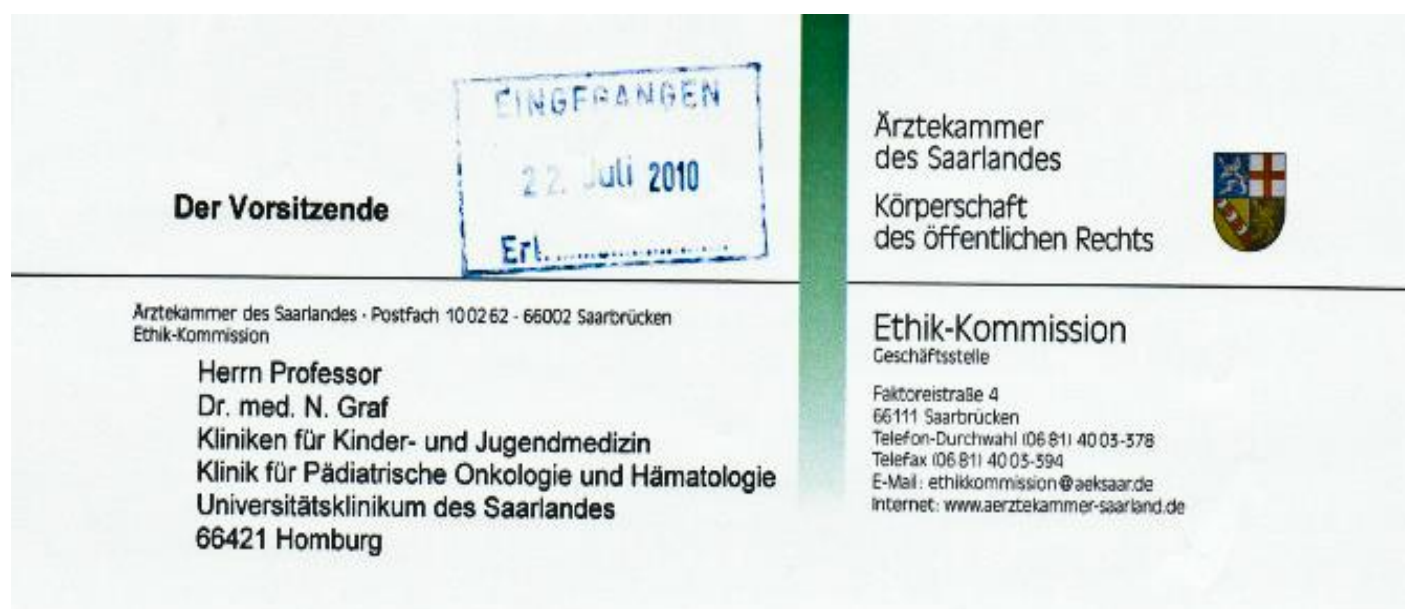

Unsere Kenn-Nr:

hr Schreben vom

Inr Zeichen:

Datum:

Prof. Schie./Sn

20. Juli 2010

Europäische Forschungsprojekte:

1. ACGT (Advancing Clinico Genomic Trials)

2. ContraCancrum (Clinically Oriented Translational Cancer Multilevel Modelling)

3. TUMOR (Transatlantic Tumor Model Repositories)

Unsere Kenn-Nr.: 104/10 (bitte stets angebent)

\section{Sehr geehrter Herr Graf!}

Die Ethik-Kommission hat sich in ihrer Sitzung am 15. Juli 2010 mit dem Antrag vom 09.06.2010, Eingang hier: 14.06.2010, befasst. Als Studienleiter präsentierten Sie die Forschungsvorhaben und standen der Kommission im Anschluss zur Diskussion zur Verfügung.

Für diese drei europäischen Forschungsprojekte sollen in aller Regel anonymisierte, jedoch auch pseudonymisierte Daten weitergegeben werden. Für die retrospektiven Daten liegt nicht in jedem Fall das Einverständnis vor, diese pseudonymisierten Daten in Forschungsprojekten zu benutzen. Der Verzicht auf das Einholen einer Einwilligung bei Zurückgreifen auf retrospektive pseudonymisierte Daten ist unter bestimmten Voraussetzungen ethisch und rechtlich begründbar. Als Begründung für den Verzicht auf das nachträgliche Einholen einer Einwilligung gelten die in der Stellungnahme der Zentralen Ethikkommission; „Die (Weiter-)Verwendung von menschlichen Körpermaterialien für Zwecke medizinischer Forschung" vom 20. 02. 2003 unter E, Ziffer II, Punkt 8 angefuhrten Voraussetzungen (Anlage, Seite 9 und 10 von 16).

Die Ethik-Kormmssion bei der Arztekammer des Saarandes ist unter Beachtung der internationalen Richtinien der ICH, CCP.V und der 12 . Novele ANC

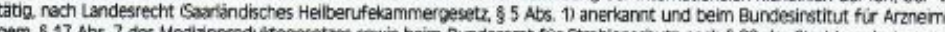
gem. 817 Abs. 7 des Mediznnroduktegesetzes sowiB beim Bundesamt fur Strahlenschutz nach $\$ 92$ der 5 trahlenschutzverordinung und nach 828 converordhumg registriert

Commerabank Sartruicken Kto.N. N. 5389200

Dt Apotheker- und Aratebank 
-2 .

Unter Bezugnahme auf $\S 2$ des Statuts der Ethik-Kommisslon

bei der Ärztekammer des Saarlandes bestehen gegen die

Durchführung der beabsichtigten Forschungsvorhaben keine Bedenken.

Wir wünschen Ihnen für die vorgesehene Aufgabe viel Erfolg und wären Ihnen zur gegebenen Zeit für die Übersendung eines Abschlußberichtes dankbar

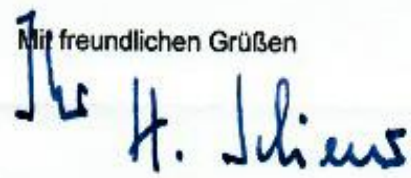

San.-Rat Prof. Dr. Schieffer

$\begin{array}{ll}\text { Mitglieder der Ethik-Kommission } \\ \text { Vorsitzender: } & \text { San.-Rat Prof. Dr. med. H. Schieffer } \\ \text { Stelly. Vorsitzender: } & \text { Prof. Dr. med. G. Rettig-Stürmer } \\ \text { Mitglieder: } & \text { Prof. Dr. med. K.-H. Altemeyer } \\ & \text { Prof. Dr. med. J. Wilske } \\ & \text { Prof. Dr. Dr. h. c. W. Schmidt } \\ & \text { Dr. med. U. Klefaber } \\ & \text { Just.-Rat Prof. Dr. jur. E. Müller } \\ & \text { Prof. Dr. rer. nat. U. Feldmann } \\ & \text { Carola Peters, MScN } \\ & \text { Prof. Dr. V. Flockerzi } \\ & \text { Prof. Dr. W. Hoffmann } \\ & \\ & \text { Dr. med. I. Lelieur }\end{array}$

Intemist/Kardiologe/Intensivmedizin Internist/Kardiologe/Intensivmedizin Anasthesist

Rechtsmediziner

Gynäkologe u. Geburtshelfer

Allgemeinarzt/Psychotherapie

Jurist, zum Richteramt befăhigt

Med. Biometrie+Informatik, Epidemiologie

Leiterin des Schulzentrums am

Universitătsklinikum des Saarlandes

Pharmakologe (abwesend)

Pädiater

Leiterin der Geschättsstelle

Figure 38: Ethical Approval from the 'Ärztekammer des Saarlandes'

Ethical Approval given by the Ethical Committee of the 'Ärztekammer des Saarlandes' at the $20^{\text {th }}$ of July 2010 for the ContraCancrum- (Clinically Oriented Translational Cancer Multilevel Modelling; project reference number: 223979; http://contracancrum.eu/) and TUMOR-Project (Transatlantic Tumour Model Repositories: project reference number: 247754; http://tumor-project.eu/) that were funded under the $7^{\text {th }}$ Framework Program of the European Commission. 


\section{Curriculum Vitae}

\section{PERSÖNLICHE DATEN}

Name: Jonathan Nicolas Zepp

Geburtsdatum: $\quad$ 15.01.1986

Geburtsort: Mainz

STUDIUM UND SCHULBILDUNG

Studium:

07/2012 - 03/2013 Promotionssemester an der medizinischen Fakultät der Universität des Saarlandes

$06 / 2012$

Approbation als Arzt

$05 / 2012$

Zweiter Abschnitt der Ärztlichen Prüfung (Note „gut")

09/2007-08/2008 ERASMUS Studium an der Universidad de LaLaguna, Spanien

$08 / 2007$ Erster Abschnitt der Ärztlichen Prüfung (Note „gut")

09/2005 Beginn des Hochschulstudiums der Humanmedizin an der medizinischen Fakultät der Universität des Saarlandes

Schulbildung:

03/2005 Allgemeine Hochschulreife (Note: 1,7)

$09 / 1996-03 / 2005$ Gutenberg-Gymnasium, Mainz

$09 / 1992-07 / 1996$ Heinrich-Mumbächer-Grundschule, Mainz 